\title{
MULTI-FREQUENCY ELECTRICAL IMPEDANCE METHOD FOR DETECTION OF VIABLE MICRO-ORGANISMS, THEIR QUANTIFICATION AND THEIR CHARACTERIZATION
}

A Dissertation presented to the Faculty of Graduate School

University of Missouri

In Partial Fulfillment

of the requirements for the Degree

Doctor of Philosophy in Biological Engineering

by

SACHIDEVI PUTTASWAMY

Dr. Shramik Sengupta, Dissertation Supervisor

DECEMBER 2013 
C Copyright by Sachidevi Puttaswamy 2013

All Rights Reserved 
The undersigned, appointed by the dean of the Graduate School, have examined the Dissertation entitled

\section{MULTI-FREQUENCY ELECTRICAL IMPEDANCE METHOD FOR DETECTION OF VIABLE MICRO-ORGANISMS, THEIR QUANTIFICATION AND THEIR CHARACTERIZATION}

Presented by Sachidevi Puttaswamy

A candidate for the degree of Doctor of Philosophy in Biological Engineering and hereby certify that, in their opinion, it is worthy of acceptance.

Dr. Shramik Sengupta, Department of Biological Engineering

Dr. Shubhra Gangopadhyay, Department of Biological Engineering

Dr. Kevin Gillis, Department of Biological Engineering

Dr. Fu-Hung Hsieh, Department of Biological Engineering

Dr. Azlin Mustapha, Department of Food Science 


\section{Dedication:}

To my parents Mr. H.C. Puttaswamy and Mrs. H. Gowramma, husband and Inlaws..... 


\section{ACKNOWLEDGEMENTS}

I would like to thank all the people who have helped me personally and professionally, whose support and efforts have made this work possible. My parents have played an important role in shaping who I am as a person, without whose motivation and guidance, I would not be where I am today. I would like to thank my husband Dr. Sagar K Gupta whose constant inspiration and encouragement has helped me achieve my goals. I would also like to thank my Mother-In-Law, Mrs Seetha Kishore who has been very supportive from the start of my studies. My Sister and Brother-In-Law have also played a crucial role in inspiring me to live up to their standards. I cannot thank my closest friends enough for making me feel right at home and made my stay here a very memorable experience.

The first person I would like to thank is my advisor Dr. Shramik Sengupta for not only being an excellent guide academically, but also in helping me at a more personal level when I joined this university as an international student. He has been a constant pillar of support for me at the University, has been delightfully patient and actively encouraged me several times during my doctoral program. I would also like to thank Dr. Hsieh for agreeing to be my mentor and guiding me during my $\mathrm{PhD}$. No words can describe my gratitude towards Dr. Shubhra Gangopadhyay and Dr. Kevin Gillis for their constant support and advice regarding my research work. I am very grateful to have had constructive interaction and discussion regarding my research work that paved way to the successful completion of the life saving technology. I am very thankful for Dr. Azlin Mustafa for helping me and advising me on the different techniques. 


\section{TABLE OF CONTENTS}

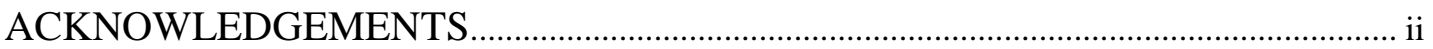

LIST OF TABLES ….................................................................................................... vii

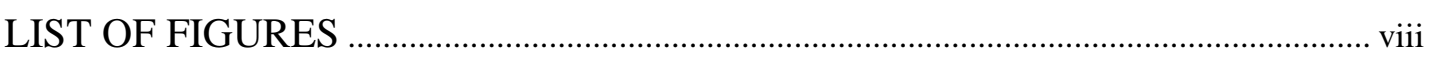

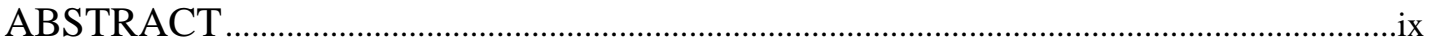

CHAPTER

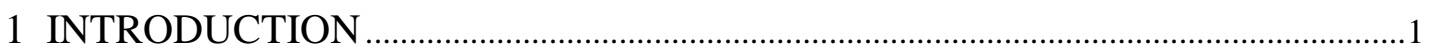

1.1 Motivation - The importance of detecting live microorganisms ......................1

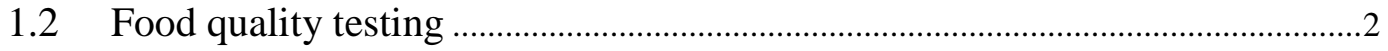

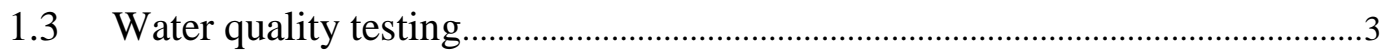

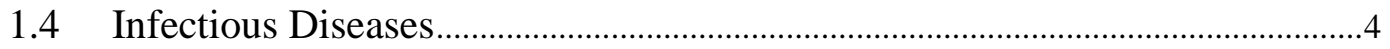

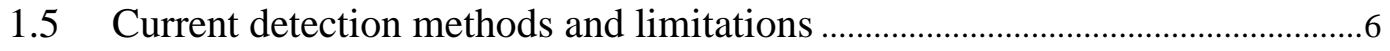

1.5.1 Traditional methods of detection ....................................................................6

1.5.2 Automated culture based methods ............................................................

1.5.3 DNA based methods ..............................................................................

1.5.4 Antibody based methods ....................................................................... 10

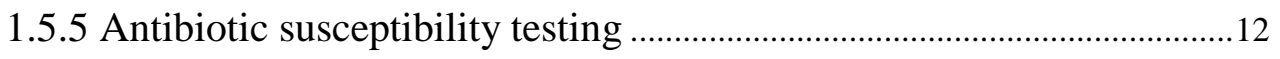

2 MULTIFREQUENCY IMPEDANCE METHOD: BASIC THEORY AND

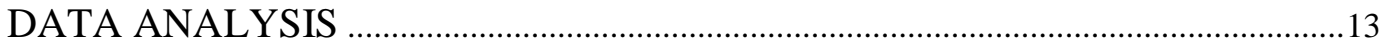

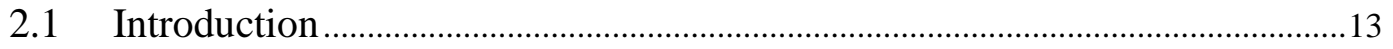

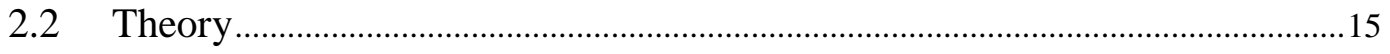

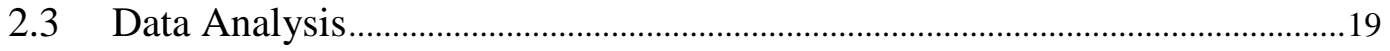

3 DESIGN AND FABRICATION OF HE MICROFLUIDIC CASSETTE ................25

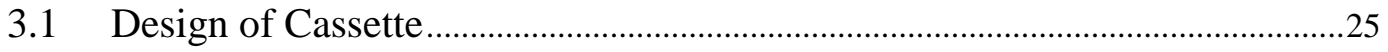

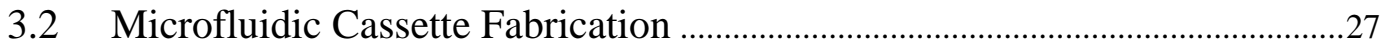

3.3 Optimization of the Exposure Time for Varying Channel Thickness and

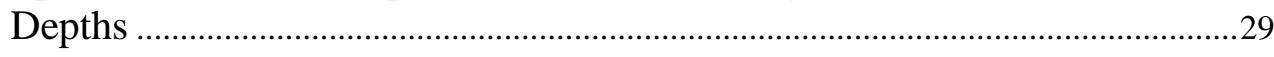

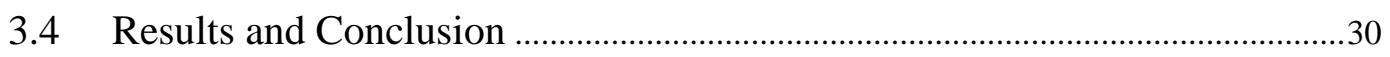

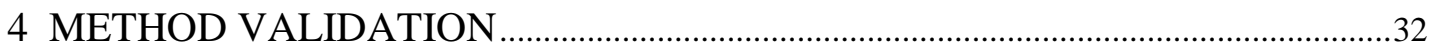

4.1 Determining Dielectric Constant of Various Solutions .........................................

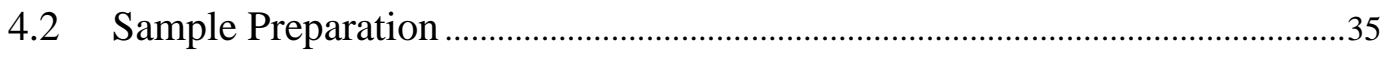

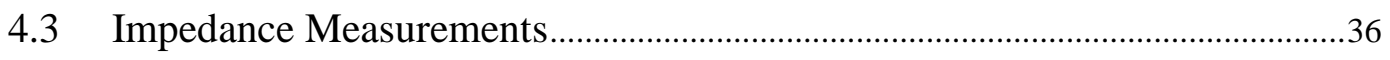

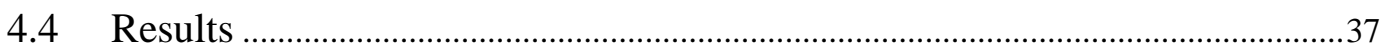


5 RAPID DETECTION OF BACTERIAL PROLIFERATION IN FOOD SAMPLES USING MICROCHANNEL IMPEDANCE MEASUREMENTS AT

MULTIPLE FREQUENCIES

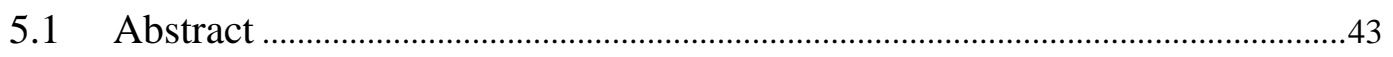

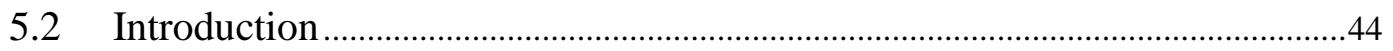

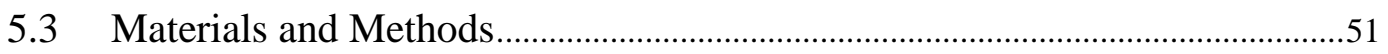

5.3.1 Sample preparation and inoculation of bacteria into samples ................51

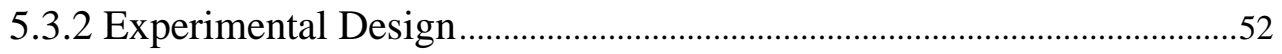

5.3.3 Electrical Measurement Technique..............................................................53

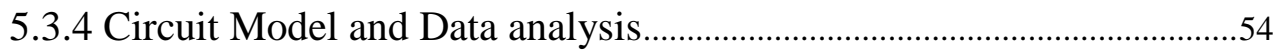

5.3.5 Establishing Times to Detection (TTDs) for Individual Samples........61

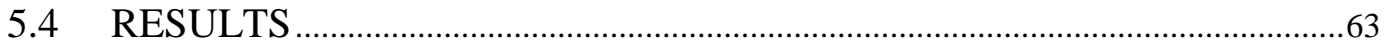

5.4.1 Ability of the Calculated CPE-T Value to Track True Bacterial Counts

5.4.2 Times to Detection (TTD) as a Function of Initial Bacterial Loads ...65

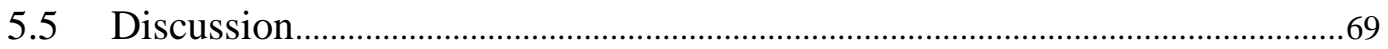

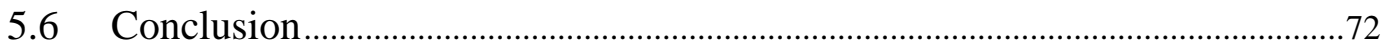

6 NOVEL ELECTRICAL METHOD FOR EARLY DETECTION OF VIABLE BACTERIA IN BLOOD CULTURES ……....................................................................

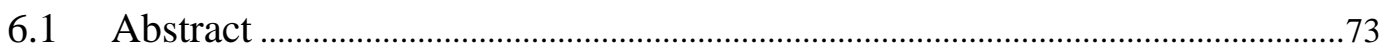

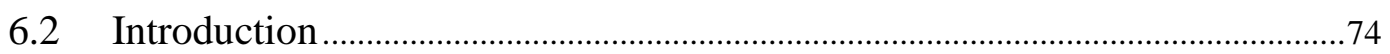

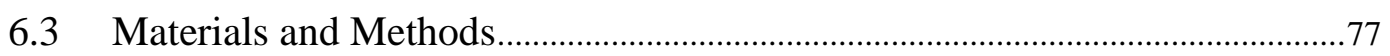

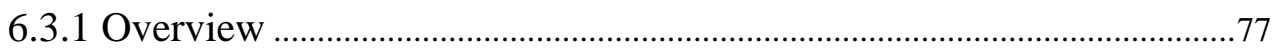

6.3.2 Preparation of the Microfluidic Cassette ................................................... 80

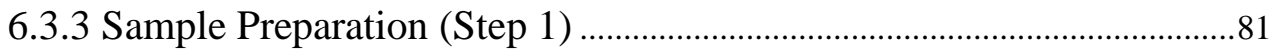

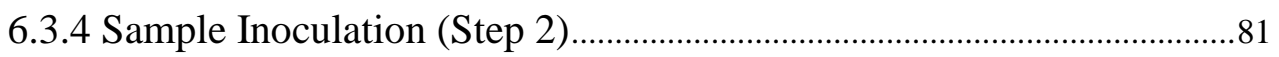

6.3.5 Blood Culture Protocol (Steps 3.1 and 3.2) ............................................ 82

6.3.6 Multi-frequency Electrical Impedance Measurements ...........................83

6.3.7 Circuit Model and Data Analysis ................................................................ 84

6.3.8 Tracking CPE-T Values, and Obtaining Times to Positivity (TTPs) 86

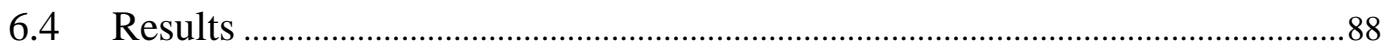

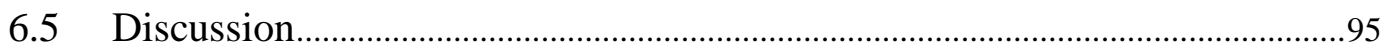


7 NOVEL ELECTRICAL METHOD FOR THE RAPID DETERMINATION OF MINIMUM INHIBITORY CONCENTRATION (MIC) AND ASSAY OF

BACTERICIDAL/BACTERIOSTATIC ACTIVITY 100

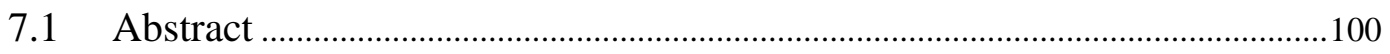

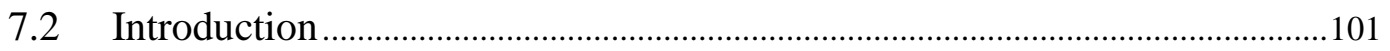

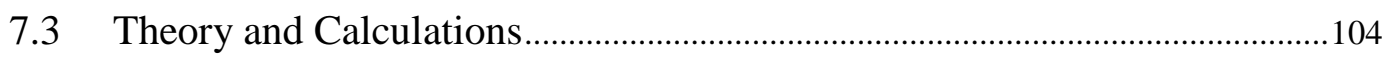

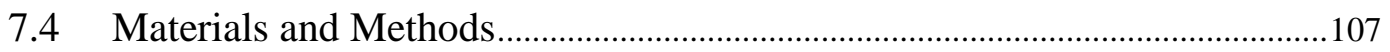

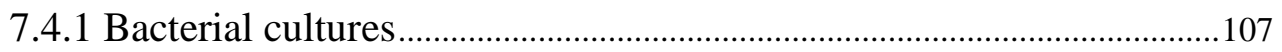

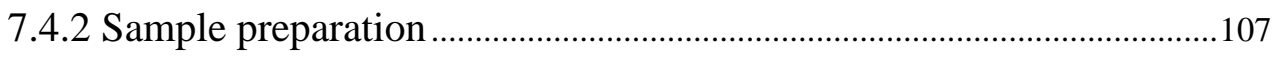

7.4.3 Multi-frequency Impedance Measurements ...........................................108

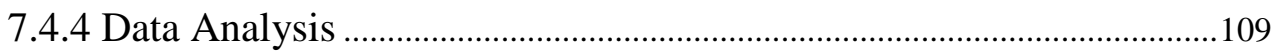

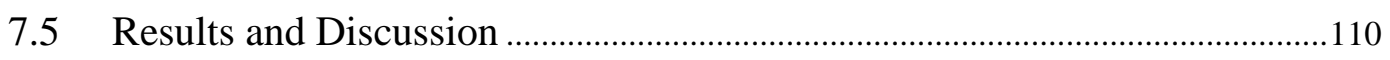

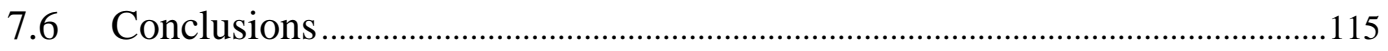

8 MULTI-FREQUENCY ELECTRICAL IMPEDANCE METHOD FOR THE RAPID DETECTION OF VIABLE MICROORGANISMS, AND THEIR RAPID QUANTIFICATION

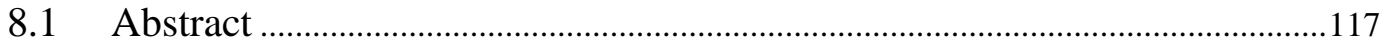

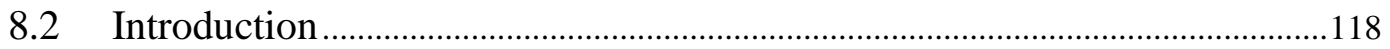

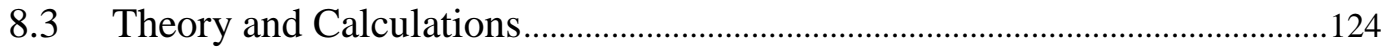

8.4 Times to Detection (TTDs) Determination ....................................................... 125

8.5 Quantifying the Number of Bacteria Present [Most Probable Number

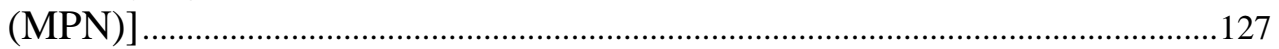

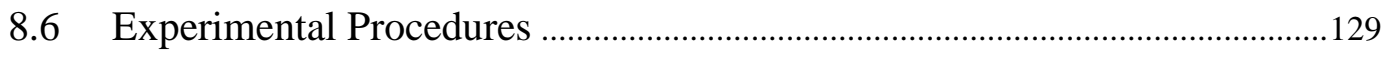

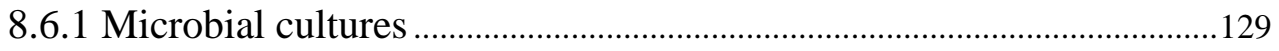

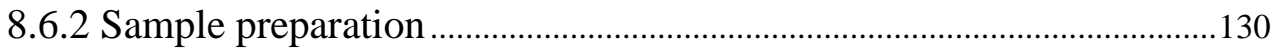

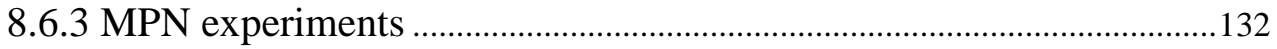

8.6.4 Multi-frequency impedance measurement and data analysis ..............133

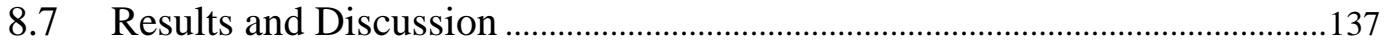

8.7.1 Rapid detection of viable microorganisms .............................................137

8.7.2 Dependence of TTD on initial load $\left(\mathrm{n}_{0}\right)$ and doubling time $\left(\mathrm{t}_{\mathrm{D}}\right)$ of the micro-organism.

8.7.3 Determining the Most Probable Number (MPN) of viable bacteria 143

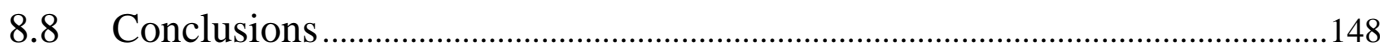

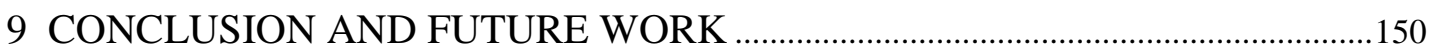




\section{REFERENCES}

VITA 


\section{LIST OF TABLES}

Table Page

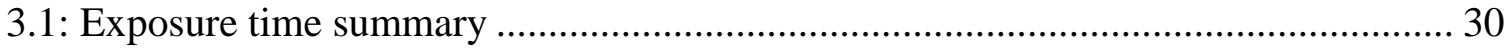

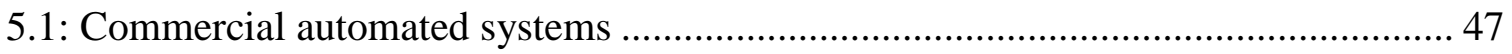

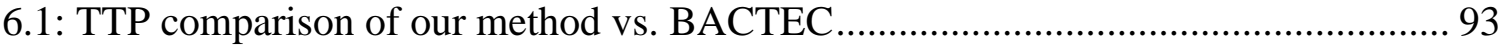

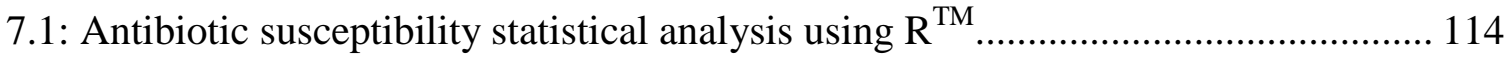

7.2: MIC value comparison by our method vs. Standard values ................................. 115

8.1: Experimental parameters used for testing microorganisms of interest ................... 131

8.2: TTDs for different initial loads of microorganisms and their calculated

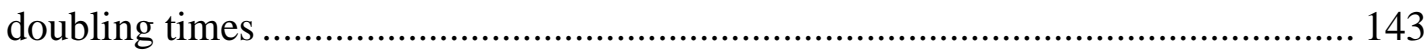

8.3: Comparison of results obtained using our method and the traditional method

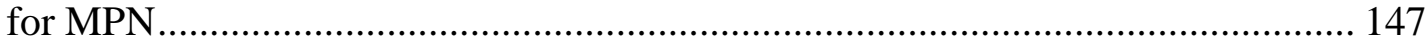




\section{LIST OF FIGURES}

$\begin{array}{lll}\text { Figure } & \text { Page }\end{array}$

2.1: Schematic and electrical representation of microchannel.................................... 17

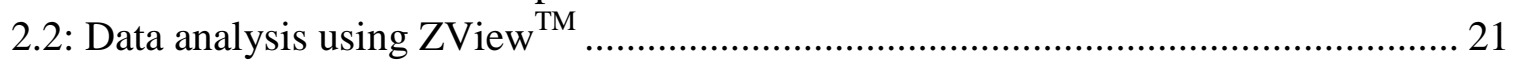

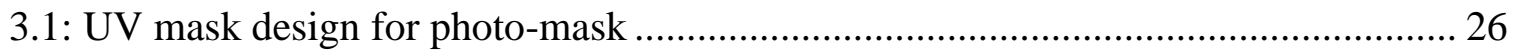

3.2: Photo-polymerization technique for cassette development ..................................... 28

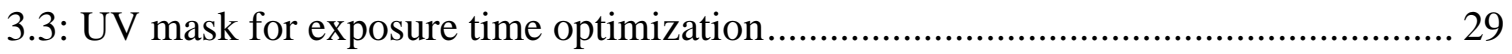

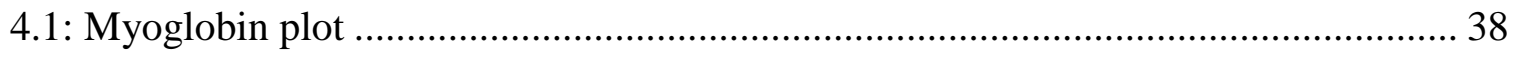

4.2: Dielectric constant plots for myoglobin(a), sucrose(b), BSA(c) and AHA(d) ......... 39

4.3: Bulk Capacitance plots of E.coli in TSB (a), Milk (b) and 2-shell model fitting for

E.coli in Milk (c) ............................................................................................ 41

5.1: Schematic and electrical representation of microchannel with equation.................. 56

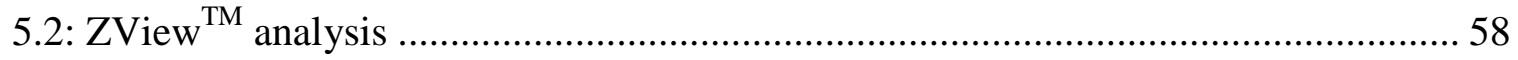

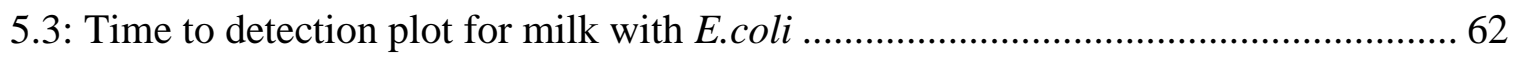

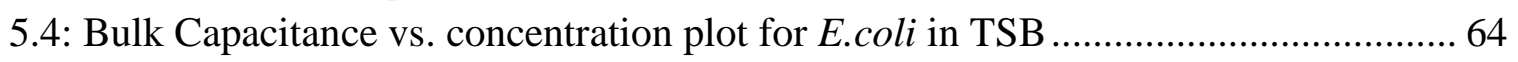

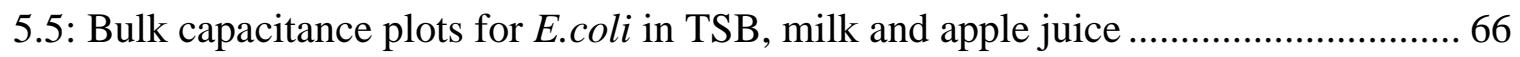

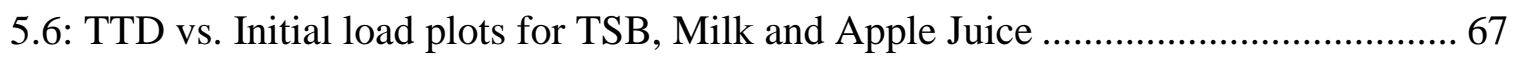

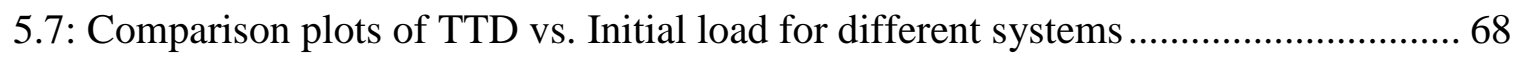

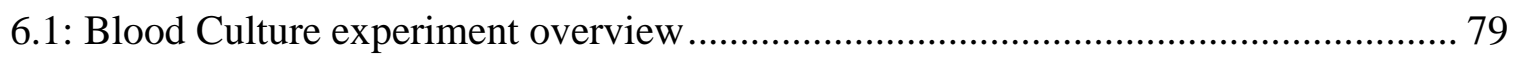

6.2: $\mathrm{ZView}^{\mathrm{TM}}$ analysis with circuit used for blood cultures ......................................... 85

6.3: Bulk Capacitance vs. time plot for E.coli in blood ............................................ 88

6.4: Plots of Bulk capacitance vs. time for E.coli in blood at different concentrations.... 90

6.5: Plot of TTD as a function of Initial load of bacteria ............................................ 92

7.1: Microfluidic channel design and equivalent circuit............................................. 110

7.2: Antibiotic susceptibility plots for bacteria in antibiotics .................................... 112

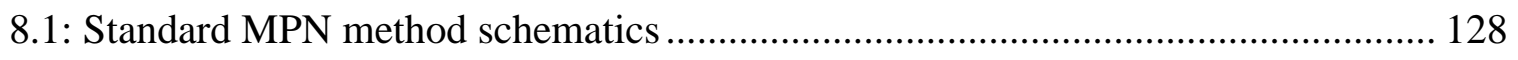

8.2: Plot of M mesophilicum bulk capacitance values over time ................................ 136

8.3: Plot of results obtained for all different microorganisms ..................................... 138

8.4: TTDs vs. Initial loads for all microorganisms used.......................................... 140

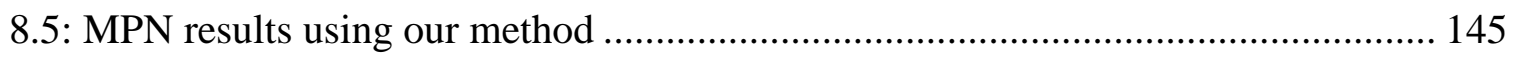




\title{
MULTI-FREQUENCY ELECTRICAL IMPEDANCE METHOD FOR DETECTION OF VIABLE MICRO-ORGANISMS, THEIR QUANTIFICATION AND THEIR CHARACTERIZATION
}

\author{
Sachidevi Puttaswamy \\ Dr. Shramik Sengupta, Dissertation Supervisor
}

\begin{abstract}
Despite advances in DNA based technologies and molecular methods, there are still several drawbacks to these methods and hence, there is a need for development of a new technology for microbial detection and quantification. We present here a novel method for detecting the presence of viable microorganisms in various suspensions ranging from bacterial growth media, to food substrates such as milk and apple juice, water and complex suspensions like blood along with their quantification and characterization.

The underlying principle for our method of detection of microorganisms is based on the fact that in the presence of an AC electric field, the intact/viable bacterial cell membrane becomes polarized. This polarization leads to the buildup of charges across the intact membrane and hence, these viable microbial cells behave like electrical capacitors. An increase in the number of microorganisms due to proliferation thus results in a corresponding increase in the "bulk capacitance" of the suspension containing bacteria, due to an increase in the total amount of charge stored in the interior of a suspension. However, this increase cannot be directly measured since the capacitance of the solid-
\end{abstract}


liquid interface ("double layer" which is usually $~ 1000 \mathrm{X}$ larger than the bulk capacitance) in effect "screens" the former. In the work presented here, we introduce a method that is able to discern such changes with high sensitivity and robustness.

We also demonstrate the ability of our method to a) Monitor food quality/safety by detecting bacterial proliferation in "real world" liquid food samples like milk and apple juice b) Quantify the bacteria in a sample by estimating the MPN of the viable bacteria c) Detect presence of viable bacteria in blood for diagnosis of Sepsis d) Determine the Minimum Inhibitory Concentrations (MIC) and assay for bactericidal/bacteriostatic activity of various bacteria e) Extend the application to detect presence of other microorganisms such as yeast and mold. 


\section{CHAPTER 1}

\section{INTRODUCTION}

\subsection{Motivation: The importance of detecting live microorganisms}

Microorganisms such as bacteria, yeast and molds can easily grow and proliferate even in the presence of minimal nutrients. They can easily contaminate water and food products when they come in contact with them, if the samples are not handled and treated appropriately. These contaminated products, if consumed by the consumers, will lead to food and water borne illnesses. If the contaminated water is being used by pharmaceutical industries for drug production, these microbes can find their way into the final products as well. They can also easily enter the blood stream through small cuts or exposures and multiply rapidly, overwhelming the body's immune system leading to septicemia and infections. In these and several other cases, rapid detection of microorganisms is very important so as to prevent health risks associated with the consumers and also to reduce economic and healthcare costs. In all of the instances described above, one expects to find dead microorganisms, their DNA and cell materials to be present in the samples as the food products and water are typically sterilized/pasteurized prior to its use which kills most microbes while some may survive. Even in the blood stream small infiltration with microbes are taken care of by the immune system, leaving behind dead cells, their DNA and antigens which do not pose any threat to us. Thus, the detection systems developed in 
these situations need to detect the presence of live microorganisms in the samples without being affected by the presence of dead cells and other non-microbial cells.

\subsection{Food quality testing}

In food industries, there are very strict limitations with respect to the bacterial presence in consumable food products. Procedures such as pasteurization, thermal and chemical procedures are commonly used in food industries to eliminate bacteria from their products. However, there is a possibility that some bacteria will survive these initial processes (Goverd et al. 1979 ; Juffs and Deeth 2007) or may be introduced into the food during additional sample processing or handling (Drake et al. 2002 ; Kirov et al. 1993). These surviving bacteria can then multiply in the food product over the next several days, leading to food spoilage and/or food borne illnesses depending on the type of bacteria. There are 76 million food-borne illnesses that occur per year in the USA alone leading to thousands of deaths (Mead et al. 2000) and an economic impact of $\sim \$ 14$ billion per year (Buzby and Roberts 1997).

To prevent this, food products are regularly tested to detect the presence of any live bacteria. Here, the detection of live or viable bacteria is of importance, as these food products carry dead bacteria which have been killed during the treatment processes and do not pose any threat to food spoilage or consumers' health. The current methods of detection used by the industries are slow, typically taking about $\sim 2-3$ days to get the results, by which time many of these products are already shipped to the market and consumed by its consumers. This not only affects the health of its consumers but also 
leads to huge economic losses to the industries due to product recalls. An interesting fact to note here is that the presence of even as few as $1-10$ viable pathogenic bacteria in the food products is sufficient to cause significant deterioration of health in the consumers. In fact, many regulatory authorities have strict regulations on the allowable number of bacteria in a given product. The US Department of Agriculture (USDA) has an allowable bacterial limit in ground beef of $<1 \mathrm{CFU} / 25 \mathrm{~g}$ of beef (Teratanavat and Hooker 2004). The US pasteurized milk ordinance requires "Grade A" pasteurized milk to have a total viable bacterial count of $\leq 20,000 \mathrm{CFU} / \mathrm{ml}$ and a coliform count of $\leq 10 \mathrm{CFU} / \mathrm{ml}$ (Hayes and Boor 2001). Product recalls in these cases lead to 10 million pounds of meat and poultry spoilage (Teratanavat and Hooker 2004) and 19 million pounds of milk wastage each year (Doyle 2007). In these cases, there is a need for a system that is able to rapidly detect the presence of viable microorganisms even when they are present at low initial concentrations and to rapidly and accurately quantify the number of microorganisms present in a given sample.

\subsection{Water quality testing}

Apart from the food industries, the quantification of the microorganisms is also important in situations such as water quality testing and monitoring. The Environmental Protection Agency (EPA) specifies that the concentration of viable coliform bacteria should not exceed $500 \mathrm{CFU} / 100 \mathrm{ml}(5 \mathrm{CFU} / \mathrm{ml})$ for primary contact recreational waters (such as lakes and beaches, where humans are fully immersed) and $8 \mathrm{CFU} / \mathrm{ml}$ for secondary contact recreational waters (such as streams where they will be immersed only 
partially) (Anderson and Davidson 1997). In the mean time, a particular beach or resort will not be immediately aware of increased risks to users. On the other hand, after the risk has been identified (and actions such as chlorination undertaken), it takes a similarly long time to ascertain that the bacterial counts are back to acceptable levels. The former leads to spread of disease among users, and the latter leads to economic losses (not only due to the loss of business on the days closed, but also through an increase in the negative perception of the area or resort). In 2008, the number of closing and advisory days at ocean, bay and Great Lakes beaches topped 20,000 for the 4th consecutive year (Dorfman et al. 2009).

Water quality testing is also important in pharmaceutical plants where there is no tolerance for the presence of live microorganisms after water treatment procedures (US Pharmacopeial Convention 2004). In most water facilities the water samples are tested regularly (daily or on weekly basis) using the MPN method. However, these current tests take about $2-3$ days to obtain results during which time, the water facilities are still being used risking its users with potential health hazards (Dorfman et al. 2009). In these and several other cases, rapid quantification of the microorganisms using better methods of detection is of most importance.

\subsection{Infectious Diseases}

It has been estimated that the number of deaths due to various infectious diseases, caused by pathogenic microorganisms such as bacteria, fungi etc, was $\sim 15$ million worldwide in the year 2002 (Madigan et al. 2006). Infectious diseases have also been 
ranked as the second most leading cause for deaths, after cardiovascular diseases, accounting for $23 \%$ of worldwide deaths according to the WHO report 2008 (Doyle 2007). According to the WHO report 2007, infectious diseases are not only spreading faster, they appear to be emerging more quickly than ever before. Nearly 50 newly discovered pathogens have emerged over the past generation. There have also been more than 1100 epidemic events worldwide in 5 years (2002-2007) (Teratanavat and Hooker 2004). Many of these infectious microorganisms can also infect the bloodstream causing septicemia. Sepsis is one of the leading causes of death infecting $\sim 751,000$ people each year and taking 215,000 lives (Angus et al. 2001).

Current automated methods of detection typically take 3 days to deem a blood sample positive and $\sim 5$ days to deem the sample negative for the presence of viable microorganisms. During this period, the patient is typically on broad spectrum antibiotics which might add to the increasing antibiotic resistance of microorganisms. Also, it has been said that every hour of delay in administering the right antibiotic (which is based on obtaining the results for the cultures) decreases the chances of survival of the trauma patients by $7 \%$ (Kumar et al. 2006). In these and several other cases, it is significantly important to develop newer techniques that are able to decrease the time taken for the detection of these pathogens. The main concern with newer methods being developed to reduce the detection time, based on molecular biology and Polymerase Chain Reaction (PCR) analysis, is that they may not be able to distinguish between the live and dead microorganisms in the blood sample. It is not uncommon to have the presence of dead microbes in blood, as the body's immune system usually takes care of minor infiltrations by microbes. Also, considering the fact that newer pathogens are constantly being 
discovered, PCR and DNA based systems may not be able to detect the presence of these newly found microbes due to the absence of the probes or markers for these pathogens. Therefore, there is a current need for a newer culture based detection system, which can detect the presence of viable microorganisms rapidly and also has the ability to determine the right antibiotic for treatment to help decrease the mortality rates associated with these infectious diseases.

\subsection{Current detection methods and limitations}

There are presently several ways to detect bacteria in which can be broadly classified into three broad classes: (a) traditional methods such as plate cultures and biochemical assays, (b) DNA and antibody based methods, often involving micro/nano particles and fluorescence, (c) other "automated" techniques that rely on monitoring the effects of bacterial metabolism on the medium. Of these, traditional methods are the most extensively used, and often serve as the standard to which other techniques are compared.

\subsubsection{Traditional methods of detection}

The most standard and traditional method used for detecting the presence of these pathogenic bacteria is the plate culture technique, developed in the late 1800's. The standard procedure involves serial dilution of the sample over a few orders of magnitude, plating a small volume $(0.1 \mathrm{ml})$ of the diluted samples on petri plates containing nutrient agar medium. When plated samples are incubated over time (typically $12 \mathrm{hrs}$ to $48 \mathrm{hrs}$ ) at a desired temperature, visible colonies appear on the surface of the plates and the number 
of colonies can be counted. These counts are used to determine the concentration of bacteria in the original sample based on the number of dilutions made.

Plate count method is a "Gold Standard" and an accurate method for bacterial detection. It is still tedious, material and labor intensive to run multiple samples. Their times to detection ranges from overnight growth to weeks depending on the type of the organism and medium used (Peplow et al. 1999). These inherent limitations of the plate count method led to the development of newer methods of detection that use DNA, antibody and other macro/micro culture based methods. These methods are aimed at decreasing the overall time taken for detection of microorganisms and to make it automated.

\subsubsection{Automated culture based methods}

Most of the current automated methods for microbial detection are based on culture based methods. These systems detect the presence of microorganisms based on their metabolism. Viable microorganisms metabolize in the presence of nutrient media when they are allowed to incubate in media. They take in sugar, proteins, oxygen and other metabolites in the media, metabolizing it, and during the process releases acids, carbon dioxide and other compounds. This consumption or release of the metabolites into the media changes their media properties such as $\mathrm{pH}$, conductivity, and dissolved gas concentrations. Once a significant change in one of these properties is observed by a particular detection method, the sample is considered to be positive for the presence of pathogens. 
There are currently several systems that have been developed taking advantage of these changes in medium properties for pathogen detection which include: RABIT ${ }^{\mathrm{TM}}$ (Don Whitley Scientific Ltd) (Madden and Gilmour 1995a), Malthus systems (Malthus Instruments Ltd.) which measures electrical conductance of the media (Dalgaard et al. 1996); ESP $^{\circledR}$ culture system (TREK Diagnostic systems) which measures change in oxygen tension (Morello et al. 1994); Coli-Check ${ }^{\mathrm{TM}}$ which measures the change in $\mathrm{pH}$ due to metabolism; Bactometer ${ }^{\mathrm{TM}}$ (Bactomatic Ltd.), which uses a impedance based method (Zafari and Martin 1977) and Bactec ${ }^{\mathrm{TM}}$ (Becton Dickinson) that detects the amount of radio-labeled carbon dioxide released (Riedel et al. 2011).

However, the amount of metabolite processed by an individual bacterium is extremely small. Based on our knowledge that the specific oxygen consumption rate for E. coli is $20 \mathrm{mmol}$ of oxygen per hour per gram (dry weight) of bacteria (Andersen and Von Meyenburg 1980b) and a typical bacterium has a dry weight $\sim 10^{-12} \mathrm{~g}$ (Borsheim et al. 1990b), we estimate that one bacterium consumes only $2 \times 10^{-14}$ moles of oxygen in one hour. Hence, there has to be a sufficiently large number of bacteria present (either $a$ priori or arising due to proliferation from the smaller number initially present) before the signal generated (change in the material property of the suspension) can be effectively measured. If the bacterial count in the original suspension happens to be small (1000 $\mathrm{CFU} / \mathrm{ml}$ or lower), one must wait for cells to proliferate to an appropriately high number (often $\sim 10^{6} \mathrm{CFU} / \mathrm{ml}$ or greater) before a discernible change in the physical properties of the medium (such as $\mathrm{pH}, \mathrm{O}_{2} / \mathrm{CO}_{2}$ concentration, conductivity etc) can be noticed. Thus, for low initial loads $(<1000 \mathrm{CFU} / \mathrm{ml})$, current commercial automated systems take almost as long as the plate-cultures (overnight or longer) to provide the desired result. 
In the recent past, there have been efforts to increase the ease of handling, cut costs, and most importantly, reduce times to detection (TTD) by using microfluidic systems to miniaturize the automated methods. Bashir and coworkers have developed chip-based micro-devices in which $\mathrm{pH}$ and impedance are monitored in order to detect bacterial metabolism (Gomez et al. 2002; Yang et al. 2005), and various additional modifications like the use of interdigitated microelectrodes (Yang et al. 2004a; Yang et al. 2004b), and arrays of microelectrode based biosensors (Radke and Alocilja 2004, 2005) have been tried in order to increase the sensitivity of measurements (with respect to conventional electrodes), and thus, further decrease the TTD. While these efforts were successful (in the sense that their TTDs are lower than those of the commercially available devices), they continue to be limited by the amount of time it takes for the bacterial metabolism to significantly alter the composition of the medium when bacterial loads are low. Bashir and co-workers attempted to overcome this drawback by concentrating the bacterial cells from dilute samples to a small volume by using dielectrophoresis (DEP) prior to culture, and then detecting changes in medium composition as before (Gomez-Sjoberg et al. 2005). While the culture time needed for detection was reduced, one needs to take into account the time needed for concentration using DEP (an additional 2-3 hours) as well to get effective TTDs. Again, while successful, the actual method of detection still relies on bacterial metabolism, with its inherent limitations (as discussed earlier).

\subsubsection{DNA based methods}

DNA based methods overcome many of the disadvantages of the traditional methods. They are rapid, require less reagents and labor, and are able to identify the 
species of the bacteria present relatively easily and accurately. These types of systems utilize microbial nucleic acids, fluorescence based assays etc.

Some of the technologies that have been developed and their potential limitations are discussed here: DNA probe assays - utilizes the ability of DNA to hybridize, by labeling the DNA probes with florescence or other labels and using them to specifically target the DNA of the bacterial cells to be detected. However, these systems also have a high detection threshold $\left(\sim 10^{4}-10^{5}\right.$ cells) (Swaminathan and Feng 1994) and thus, require an enrichment process before the actual detection is conducted. Apart from that, since these techniques uses DNA, it cannot really distinguish between live and lead microbes. In-vitro DNA amplification (PCR) - is a procedure that amplifies an isolated target DNA from the sample many folds using specific DNA probes which helps detect very small quantities of target DNA and in turn the microorganism from the sample (Brian et al. 1992). This method of detection is highly sensitive, simple to operate and rapid. It requires a lot of pre-processing of the samples to eliminate the components that can inhibit PCR. Most importantly, since most PCR based methods detect presence of DNA, it cannot distinguish between the live and the dead microorganisms in the samples as it is common for many of the samples to contain dead bacterial DNA.

\subsubsection{Antibody based methods}

Immunoassays are also one of the commonly used methods for pathogen detection and efforts have been made to miniaturize counterparts of the same. Immunoassays and protein based assays take advantage of the specificity of formation of an antibody-antigen 
complex which is a result of the fact that such antibodies are produced based on specific antigens on the pathogens.

Immunoflorescent assays - utilize antibodies labeled with a florescent molecule which can be targeted to specific bacteria in clinical samples for detection and identification (Soini and Hemmilä 1979). These systems even though are rapid techniques, are still expensive, have low specificity and require trained laboratory personnel. Enzyme-linked immunosorbent assay (ELISA) - are commonly used in diagnostics and have been tailored to a wide variety of detecting targets. They utilize labeled antigens or antibodies and the interactions between the two to determine the presence of specific microorganisms/pathogens (Kemeny and Challacombe 1991). These systems are also very specific which are both an advantage and a disadvantage as they can be used to detect specific microbes but uses polyclonal antibodies which may lead to false positives. These systems also have low sensitivity needing a higher concentration of bacteria to start with- $\sim 10^{5}-10^{6}$ (Swaminathan and Feng 1994). Immunomagnetic separation - uses magnetic particles carrying antibodies against bacterial antigens on their surface to separate the bacteria from the samples (Skjerve et al. 1990). This method is a fast method of detection, but can result in false positives and also as with any other antibody based technique, one needs to specifically design the antibodies for different microorganisms but these antibodies might not be able to detect a broad array of microorganisms.

It is thus important to develop a method to detect the presence of viable microorganisms only, as it is the viable organisms that are a cause of concern. Due to the problems of non-specificity, sensitivity and the cost associated with antibody and DNA 
based methods, culture based methods still are more reliable and commonly used method for microbial detection, quantification and characterization.

\subsubsection{Antibiotic susceptibility testing}

Apart from these, there has also been a growing concern due to an increase in the antibiotic resistance among microorganisms. Some of the most common microorganisms that have developed antibiotic resistance include enterococci, Staphylococcus aureus (MRSA), Mycobacterium tuberculosis, Pseudomonas aeruginosa. Hence, there is currently an unmet need to develop a technology that can be used to detect the presence of viable bacteria a) Rapidly - is able to screen food quickly for quality control where it is produced or distributed (with low time to detection) b) with high sensitivity - to be able to detect bacteria in suspensions starting with a very low concentration c) with least number of false positives-is unaffected by the presence of dead or nonviable bacteria. 


\section{CHAPTER 2}

\section{MULTIFREQUENCY IMPEDANCE METHOD: BASIC THEORY AND DATA ANALYSIS}

\subsection{Introduction}

In the previous work by Sengupta et al (Sengupta et al. 2006b), an approach was proposed to detect the presence of viable bacteria in a given suspension. Because bacteria can store a lot of charge $(\sim 100 \mathrm{X}$ more than an equal volume of water) (Poortinga et al. 1999b), an increase in the number of bacteria through proliferation would lead to a measureable increase in the charge storing capacity (capacitance) of the medium, and hence the reactance (out of phase component of the impedance) between two metal electrodes in contact with the medium. Other previous researchers (Felice and Valentinuzzi 1999) also had the same idea, but they failed to detect any such change in reactance. Sengupta et al (2006), showed, using mathematical models, that this was because, given the range of $\mathrm{AC}$ signal frequencies they used $(<1 \mathrm{MHz})$ and the geometry of the system, the electrochemical interface between the electrodes and the aqueous solution (the "double layer") effectively "screened" the bulk solutions. Their models also led them to propose that we could detect increased capacitance of the bulk medium due to bacterial proliferation by (a) changing the geometry of the system (using narrow and long, capillary-like geometries as depicted in Figure 2.1, the development of which has 
been explained in detail in chapter 3; to decrease the inverse-RC time constant of the system, and (b) measuring the reactance of the system at multiple frequencies, and tracking the frequency at which a reactance minima was recorded. They showed, using the proposed method and reactance (impedance) measurements up to $1 \mathrm{MHz}$, the ability to detect an initial load of $\sim 100 \mathrm{CFU} / \mathrm{ml}$ E. coli in Tryptic Soy Broth (TSB) in about 3 hours. A major drawback with this method was that the method was temperature sensitive and small fluctuations in the temperature would result in a shift in reactance values and also since the frequencies used was $<1 \mathrm{MHz}$, it at times did not include the high frequency minima values.

In this thesis, we report the following advances from the previous report (Sengupta et al. 2006a). Firstly, we enhance the sensitivity of our electrical technique through the use of (a) high frequency impedance measurements up to $100 \mathrm{MHz}$ (b) a refined electrical model for our system that is better able to describe the behavior of the bacterial particles at these high frequencies, and (c) more rigorous data analysis that is able to distinguish changes to the overall reactance due to change in capacitance from other changes (such as change in temperature). Secondly, we demonstrate that the technique is suitable for detecting various microbial proliferations in "real world" fluid samples: (a) food substrates such as milk and apple juice and (b) blood. We also showed the ability of our method to quantify the presence of viable microorganisms in pharmaceutical water using the MPN method and also determining the antibiotic susceptibility of different antibiotics on different strains of bacteria. 


\section{$2.2 \quad$ Theory}

Bacteria are prokaryotic single celled living organisms, which consists of many substances like RNA, proteins, enzymes, etc surrounded by cell membrane. Bacterial cell membrane is a semi-permeable membrane mainly consisting of phospholipids formed into a bi-layer in which a variety of proteins, ion channels, transporters, etc. are embedded. The Cell membrane of the bacteria acts as a wall that separates the interior of the cell from the rest of the surrounding environment. Due to its fluid mosaic pattern, it allows the free movement of water, and certain uncharged molecules like oxygen, carbon dioxide and acts as a barrier for movement of charged ions like $\mathrm{K}^{+}, \mathrm{Na}^{+}, \mathrm{Cl}^{-}$etc.; hydrophilic molecules like glucose, macromolecules proteins, etc. in and out of the cell.

One of the important roles of the cell membrane is to maintain the osmotic balance in and out of the cell for its survival. Typically, there is always an unequal concentration of ions across the cell membrane of bacteria which helps maintain the osmotic balance of the cell. The concentration of potassium ions is higher inside the cell than outside and that of sodium ions is higher outside the cell. Due to this distribution of ions, the outside of the cell membrane is slightly positively charged with respect to the interior of the cell membrane.

A bacterial cell can be considered as a capacitor, because the cell membrane resists the flow of ions across it thus, acting as a dielectric material and the intracellular and the extracellular fluid around the bacterial cell membrane is very conductive, thus acting as conducting materials. Because of this, oppositely charged ions accumulate on either side of the cell membrane. When a voltage is applied to the fluid consisting of cells, the applied electric field results in the movement of oppositely charged ions on either side of 
the cell membrane in the direction of the electric field. This, in turn, leads to the accumulation of charges across the membrane; thereby, the cell now acts as a capacitor (Tewari et al. 2007).

A Capacitor is an electronic element that has the ability to store a charge in them. This can be described with the use of a pair of parallel plate conductors separated by an insulating material. Whenever a voltage difference exists between these conducting plates, there will be an electric field present in the insulating material. A typical method for transferring equal and opposite charges to a capacitor is to use a voltage source to create a potential difference between the 2 conductors. Electrons will then flow off one conductor and through the connecting wires onto the other until the potential difference between the 2 conductors is the same as that of the voltage source. This finally leaves a net positive charge on one of the plates and a net negative charge on the opposite plate. Thereby capacitors can act as elements that store charge. Capacitance is defined as the measure of a capacitor's ability to store charge and is given in (2.1) equation.

$$
\mathrm{C}=\mathrm{Q} / \mathrm{V}
$$

C - Capacitance (Farads)

Q - Charge (coulombs)

V - Voltage (Volts)

For a given area of the plates (A), separated by a distance (d) with a dielectric in between, capacitance is approximately given as

$$
C=\in\left(\frac{A}{d}\right)
$$

$\epsilon=$ permittivity $=\epsilon_{0} \epsilon_{\mathrm{r}}$ 
where $\epsilon_{0}$ is the permittivity of vacuum, $\epsilon_{\mathrm{r}}$ is the relative permittivity

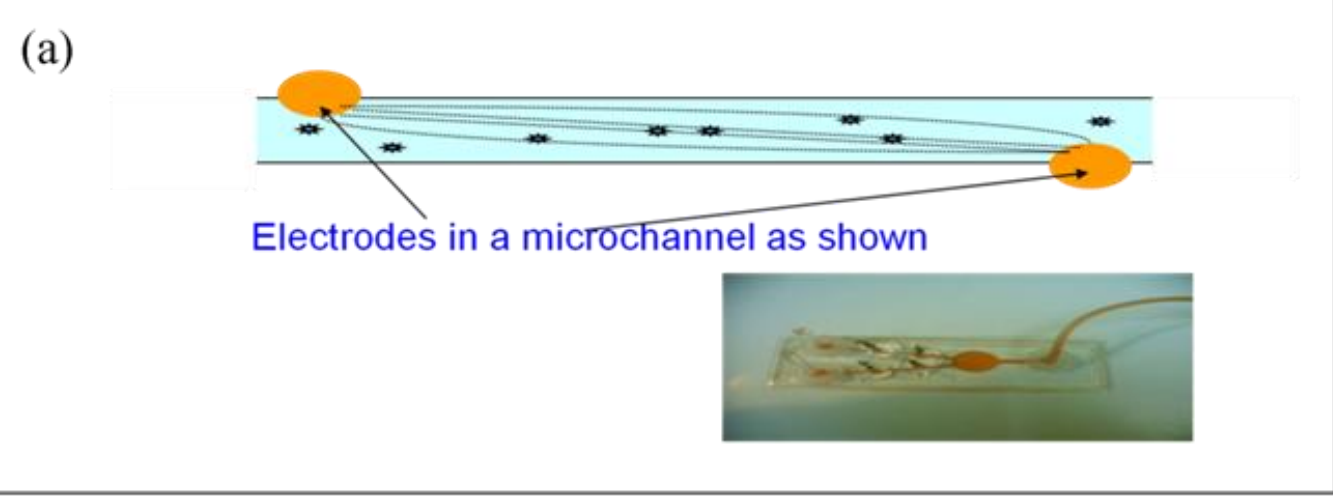

(b)
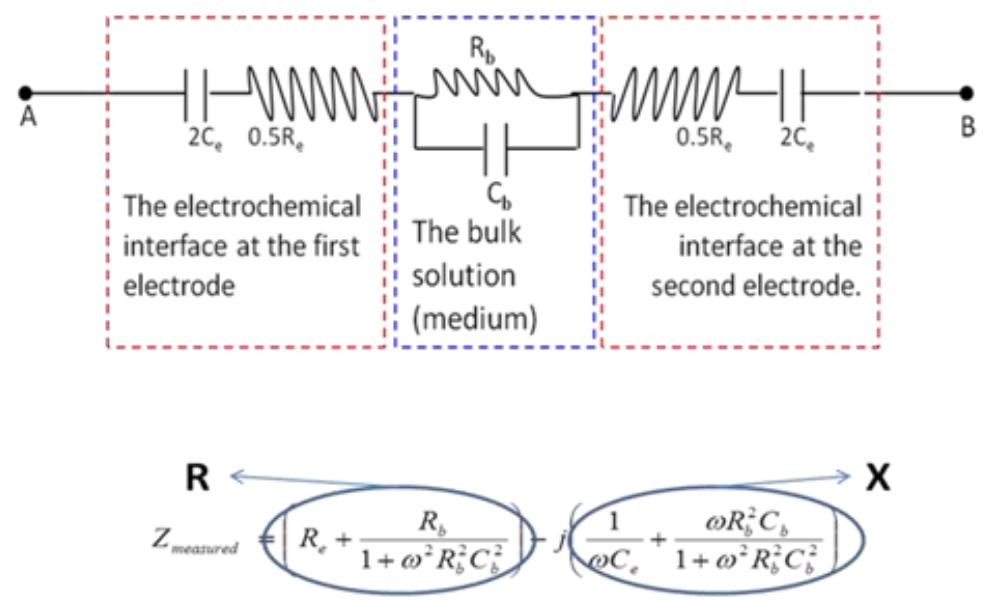

Figure 2.1: Schematic and electrical representation of microchannel

(a) Schematic representation of the micro-channel (the development of which has been explained in detail in chapter 3) with electrodes on either end loaded with suspension harboring bacteria (actual image in inset). The spatial confinement of the electrical lines of force ensure sensitivity of electrical measurements to the bacteria present 2.1(b) Equivalent circuit representation of the channel with suspension of bacteria and electrodes [ $\boldsymbol{R}_{\boldsymbol{e}}$ - Resistance at the electrode-suspension interface, $\boldsymbol{C}_{\boldsymbol{e}^{-}}$Capacitance at the electrode-suspension interface,, $\boldsymbol{R}_{\boldsymbol{b}}-$ bulk resistance, $\boldsymbol{C}_{\boldsymbol{b}}-$ bulk capacitance]. 
The Cassette shown in Figure 2.1(a) is used to take the impedance measurements of the bacterial suspension which is introduced into the channel. When the cassettes are loaded with an aliquot from the sample being investigated, the suspension fills the microchannels along which electrodes are positioned as shown in Figure 2.1(a). An Agilent 4294A Impedance Analyzer is used to measure the electrical impedance between the electrodes at multiple (>500) frequencies between $1 \mathrm{kHz}$ to $100 \mathrm{MHz}$. The instrument measures the magnitude and phase of the $\mathrm{AC}$ current that flows through the suspension upon the application of a sinusoidal AC voltage of 500mV (peak-to-peak), and calculates the Impedance (resistance and reactance) from the measurements. Since the current is not in-phase with the applied sinusoidal voltage, the Impedance, which can be considered as the AC analog of the DC resistance, has both an in-phase component called the resistance $(\mathrm{R})$, and an out-of-phase component called the reactance (X). It is typically represented as a complex number and is shown in Equation 2.3

$$
\mathrm{Z}=\mathrm{R}+j \mathrm{X}
$$

$$
\text { where } j=\sqrt{-1}
$$

Alternatively, the impedance can also be represented completely by its magnitude $(|Z|)$ and its phase angle $(\Theta)$. The magnitude and phase angle, respectively, of the impedance, are related to the resistance and reactance by Equations 2.4a and 2.4b.

$$
\mathrm{Z}=\sqrt{\left(R^{2}+X^{2}\right)}
$$




$$
\theta=\operatorname{Tan}^{-1}\left(\frac{X}{R}\right)
$$

The impedance analyzer measures impedance by measuring the resistance $(R)$ and reactance $(\mathrm{X})$ for each sample, over the frequency range of $1 \mathrm{kHz}$ to $100 \mathrm{MHz}$ and hence generates the data set containing the values of $\mathrm{R}$ and $\mathrm{X}$ at multiple ( $>500$ ) frequencies.

\subsection{Data Analysis}

In previous work (Sengupta et al. 2006b), Sengupta et al. (2006) had proposed that a system consisting of an aqueous solution in a microfluidic channel with electrodes on either end (Figure 2.1(a)), can be represented by an equivalent electrical circuit as shown in Figure 2.1(b). As seen, this takes into account the existence of electrochemical "double layers" on the surfaces of charged metal electrodes. Hence, the net measured impedance $\left(\mathrm{Z}_{\text {measured }}\right)$ is, as shown by the accompanying equation, affected by not only by the presence of conductive and capacitive (charge-storing) elements in the bulk, but also by such elements present at the electrode-solution interface. In fact, the interface capacitance is typically more than 1000 times greater than the bulk capacitance, as a result of which previous investigators (Felice and Valentinuzzi 1999) were not successful in measuring any changes in the latter, although it was expected that since bacteria can store charge (Poortinga et al. 1999b), their proliferation would lead to an increase in bulk capacitance. Sengupta et al. (2006) showed how the use of micro-channel geometry serves to concentrate the electrical field and raise the effective bulk resistance of the suspension, thereby allowing us to pick up signatures of increased bulk capacitance. Specifically, the 
signature that they looked at was a shift in the plot of measured reactance $(\mathrm{X}) \mathrm{v} / \mathrm{s}$ frequency $(\omega)$ in the range of $1 \mathrm{kHz}$ to $1 \mathrm{MHz}$.

Since the effects of the electrochemical double layer are less pronounced at high frequencies (as can be seen from the equation accompanying Figure 2.1(b)), we had initially assumed that obtaining readings at higher frequencies (up to $100 \mathrm{MHz}$ ) would enable us to obtain more sensitive readings using the same metric (shift in $\mathrm{X} \mathrm{v/s}$ curves). However, our experiments were unsatisfactory. Due to the increased contribution to the measured reactance from the bulk resistance at these higher frequencies, even extremely small changes in the value of this quantity (that we attribute to thermal fluctuations in our lab) caused shifts in the $\mathrm{X} v / \mathrm{s} \omega$ curve. Thus, our efforts to increase the sensitivity of our old measurement technique led to false positives. We needed a way to distinguish the true positives (shift due to an increase in bulk capacitance) from false positives (shift due to other reasons such as an increase in the bulk resistance).

To overcome this hurdle, we chose to adopt a different procedure, viz. to evaluate changes in all individual components of the circuit. To do so, we took the values of resistance $(\mathrm{R})$ and reactance $(\mathrm{X})$ measured by the impedance analyzer and fit that to our equivalent circuit model using a commercial circuit analysis software $\left(\mathrm{ZView}^{\mathrm{TM}}\right)$. This software, when given the circuit model and data set, is to be able to estimate each of the individual parameters $\left(\mathrm{R}_{\mathrm{e}}, \mathrm{C}_{\mathrm{e}}, \mathrm{R}_{\mathrm{b}}\right.$ and $\left.\mathrm{C}_{\mathrm{b}}\right)$ of the circuit. Using this technique, it was further seen that while the circuit model used earlier (Figure 2.1(b)) works well at low frequencies, it does not hold very well at higher frequencies. Replacing the bulk capacitance $\left(\mathrm{C}_{\mathrm{b}}\right)$ with a Constant Phase Element $(\mathrm{CPE})$ in the circuit (yielding the circuit 
shown in Figure 2(a) provided a much better fit to the data obtained - as shown in Figure 2.2(b).

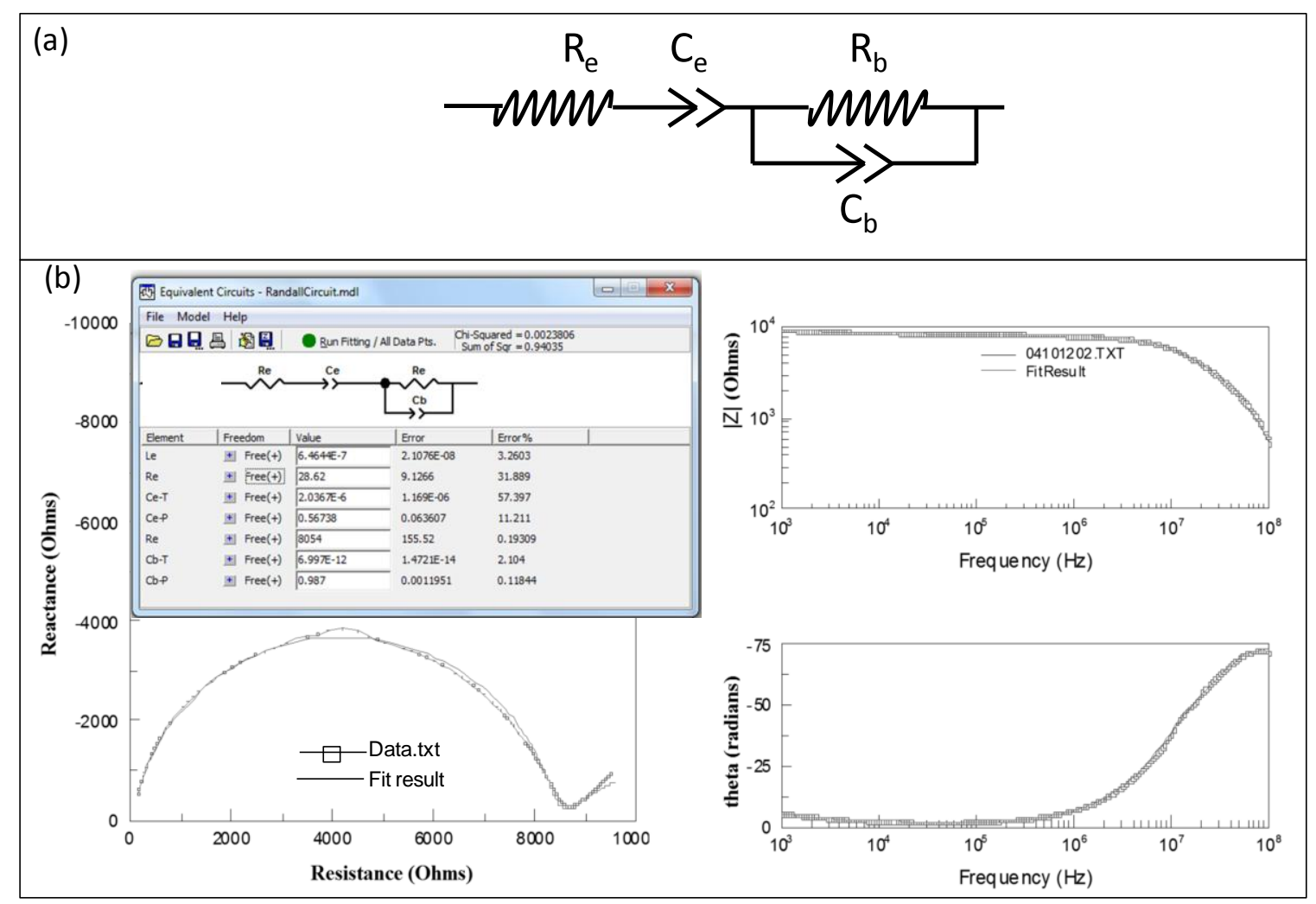

Figure 2.2: Data analysis using ZView ${ }^{T M}$

(a) Equivalent circuit diagram as used in the ZView ${ }^{T M}$ software with a Constant Phase Element used to represent the charge-holding behavior of the bulk suspension (bacteria), instead of an ideal capacitor ( $b$ ) Results obtained using ZView ${ }^{T M}$, when the data from Impedance Analyzer (red line) was fitted by the circuit model with the parameters on the right (green line). 
The CPE is a non-intuitive circuit element that replaces a capacitor in a circuit when there is some type of non-homogeneity in the system, delaying or impeding the movement of charge carriers (Barsoukov and MacDonald 2005). In more mathematical terms, the impedance of a $\mathrm{CPE}$ is given by the equation

$$
Z=0-j\left(\frac{1}{(\omega Q)^{n}}\right)
$$

Where $\omega=2$ Лf

As shown in equation 3, the impedance of the CPE is defined by two values: the magnitude component CPE-T (Q) measured in $\mathrm{Fs}^{\mathrm{n}-1}$ and the phase component CPE-P (n). If CPE-P (n) equals 1 then the equation is identical to that of a capacitor. Since it intuitively seems likely that bacteria while able to store charge, may not behave like ideal capacitors, compensating its non-ideality with CPE element seems appropriate. We could identify the need for using a CPE for our data analysis, as the arc of the Cole-Cole Plot for our impedance data was a depressed semicircle or an arc of the circle with the center somewhere below the $\mathrm{x}$-axis rather than a perfect semicircle with the center on the $\mathrm{x}$-axis as would be the case if the bacteria behaved like ideal capacitors (Meland 2007). The phenomenon of depressed semicircles has been given multiple explanations including surface roughness, varying thickness or composition of coatings, non-uniform current distributions. In short, the main reason is due to some non-homogenous nature or distribution of value of some physical property of the system. In Barsoukov and MacDonald 2005, they point out that although it is difficult to isolate the exact CPE 
behavior, it fits the experimental data well enough that it can provide a useful modeling element even if the true nature of the system in unknown.

The value of the CPE-P (n) is not a constant but is different for different sample or even samples from different batches as each sample might contain different quantities of media, cells or other components. When doing the analysis using the $\mathrm{ZView}^{\mathrm{TM}}$ software, initially the data is loaded to the software and the circuit to which it had to be fit is constructed. Then, each of the circuit parameters $\left(\mathrm{R}_{\mathrm{e}}, \mathrm{C}_{\mathrm{e}}, \mathrm{R}_{\mathrm{b}}, \mathrm{CPE}-\mathrm{T}\right.$, and CPE-P) are provided with initial guess values and the system numerically optimizes the values to obtain the best fit for the system as a whole over the range of frequencies examined. Convergence is often not guaranteed, and sometimes the system converges to a solution that is "obviously" wrong - as seen from the visual fit of the computed circuit model impedance curves (green lines in Figure 2(b)) to the actual data (red lines in figure 2(b)). Since the charge-holding behavior of individual bacteria cells presumably do not change with an increase in their number, in some (but not all) cases, the value of the CPE-P is fixed to the value obtained earlier for the same sample to help the software reach an apparently correct numerical solution. In the other cases, it is seen that the software itself arrives at a set of values for the circuit parameters in which the value of the CPE-P is extremely similar (if not identical) to that obtained previously for the same sample. It may be noted, though, that the values of CPE-P that we obtained appear to be a function of the bacterium studied and the medium used: with values of approximately $0.96,0.97$, and 0.986 for E.coli in TSB, E. coli in milk, and L. acidophilus in apple juice respectively. We do not have, at present, have any basis for predicting a-priori what the value of the CPE-P will be for a particular system. Presumably, they may even differ 
among different samples of the same product (e.g. different batches of milk, depending on the protein and fat content). 


\section{CHAPTER 3}

\section{DESIGN AND FABRICATION OF HE MICROFLUIDIC}

\section{CASSETTE}

\subsection{Design of Cassette}

The idea that the bacteria can store charge and act as capacitors has been known for a long time. But, until recently one has not been able to take advantage of it because the capacitance at the electrodes (interfacial capacitance) is much larger than the capacitance of the bulk of the solution containing bacteria. However, Sengupta et al (Sengupta et al. 2006a) designed a new microfluidic channel, with long and narrow dimensions to pick up signatures of bulk capacitance values. By increasing the length of the channels and decreasing its width, the contribution from the bulk could be made significant, so that one can measure the electrical impedance of the suspension in the channels and determine the value of bulk capacitance.

Taking into consideration this design criterion, the channels were designed to be long and narrow with electrodes on either end along the length of the channel. The electrodes were placed $1 \mathrm{~cm}$ apart, and the channel was $800 \mu \mathrm{m}$ deep and $1 \mathrm{~mm}$ wide. The microfluidic cassettes used for the measurement were fabricated using liquid phase photo-polymerization of a commercially available UV curable polymer (Loctite $363^{\mathrm{TM}}$ ). The cassette was developed using the principle of liquid photo-polymerization. The UV- 
masks were developed by creating the desired design pattern as shown in Figure 3.1. These patterns were used as UV-masks for developing the microfluidic cassettes. When exposed to the UV-light the transparent surface of the mask allows UV to pass through it thereby polymerizing any monomer underneath the mask while, the dark/black areas prevent the UV light from penetrating it, thus inhibiting the polymerization on monomer underneath its area, which can be flushed out by acetone to give the desired channel.

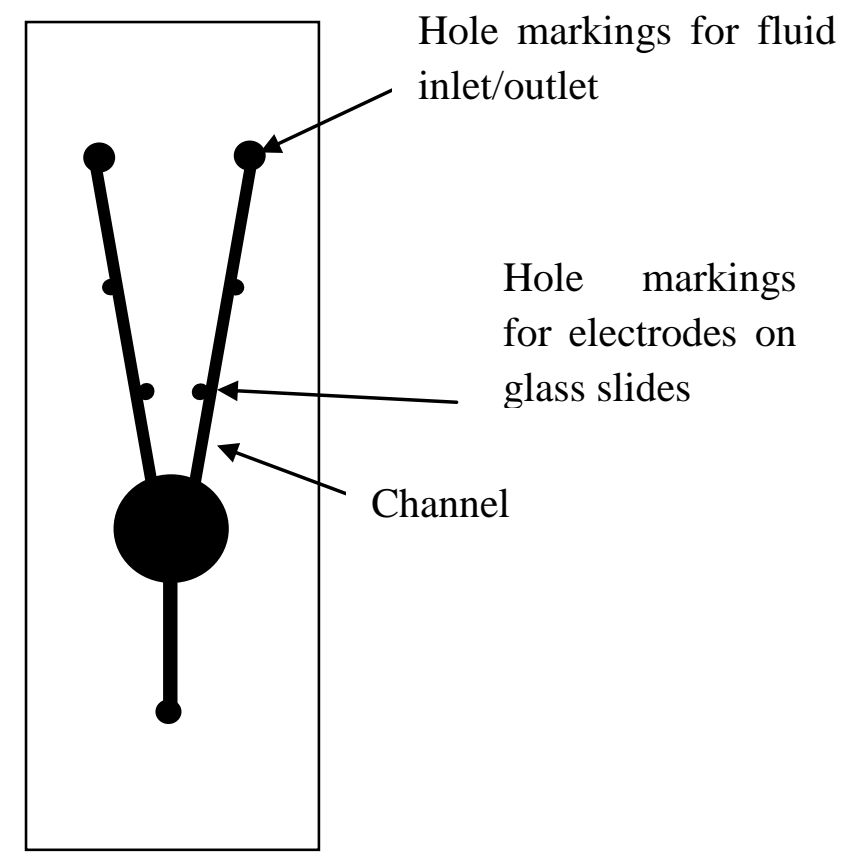

Figure 3.1: UV mask design for photo-mask Design of the UV photo-mask used for the fabrication of the microfluidic cassettes 


\subsection{Microfluidic Cassette Fabrication}

Two microscopic glass slides $(25 \mathrm{~mm}$ X $75 \mathrm{~mm})$ were used for the development of the cassettes. The detailed steps involved in the cassette development are schematically represented in Figure 3.2. One glass slide was drilled with holes as shown in the pattern in Figure 3.1 which acts as the top part of the cassette. The UV mask which was developed by printing the design on a transparency sheet was then adhered onto the surface of this glass slide such that the holes are well aligned.

Another plain microscopic slide of the same size was taken and silicon rubber adhesive back sheet of $1 / 32$ " $(800 \mu \mathrm{m})$ thick were cut into thin strips and stuck on the 4 sides of the slide to form a well. The monomer Loctite $363^{\circledR}$ light cure adhesive was applied onto this slide so that it fills in the well surface completely. The microscopic slide with UV mask is placed on top of this slide with edges aligned. This was then exposed to UV light for 22 seconds (for the specified thickness of $800 \mu \mathrm{m}$, and channel width $-1 \mathrm{~mm}$ ). Exposure time depends on the light intensity and coating depths. Immediately after exposure, the mask was peeled off and acetone is passed through the channel using a syringe to remove any unpolymerized monomer in the channel. The leftover acetone in the channel is removed through the holes using vacuum suction. The surface was then wiped clean with acetone to remove any polymer/monomer and hard baked for 2 minutes by exposure to UV light. To these hard baked cassettes, crimp-pin electrodes are glued at the holes drilled for the electrodes and the resulting cassette image is shown in figure 3.2 inset. 


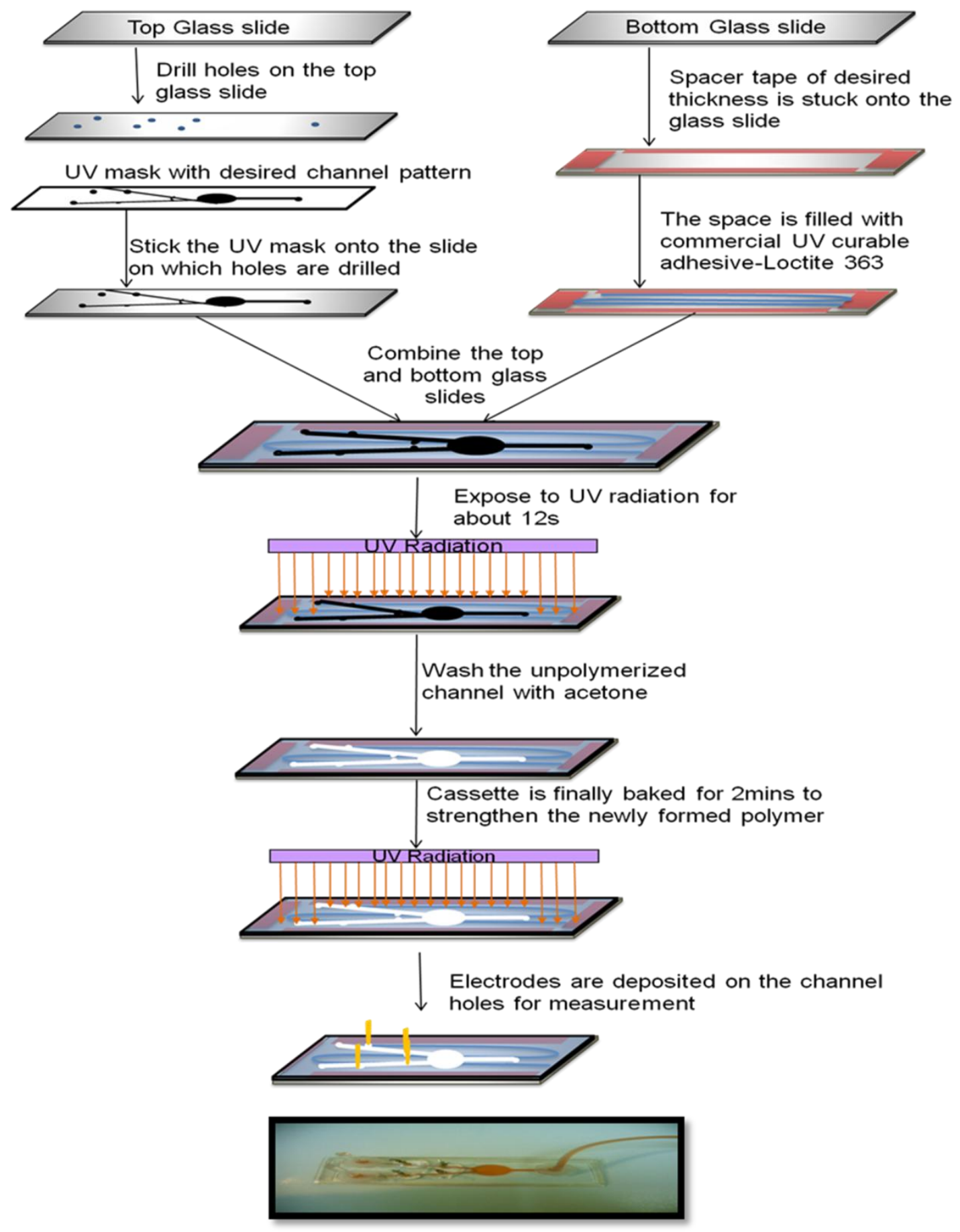

Final microfluidic cassette

Figure 3.2: Photo-polymerization technique for cassette development

Schematic representation of the photo-polymerization technique used for microfluidic cassette development 


\subsection{Optimization of the Exposure Time for Varying Channel Thickness and Depths}

The channel thicknesses can be varied by using silicon rubber sheets of different thickness. The width of the channels can be varied by varying the line width on the UV mask as shown in Figure 3.3. A simple UV mask of straight lines with different line widths were used for the purpose of optimization of exposure time.
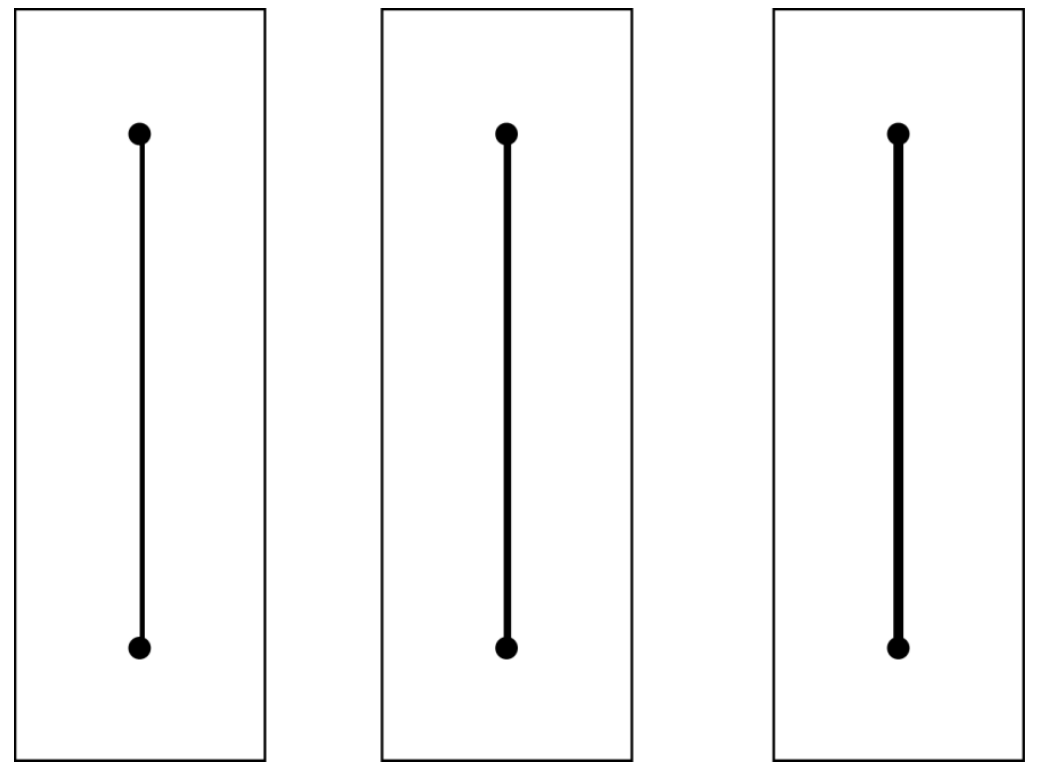

Figure 3.3: UV mask for exposure time optimization

Figure 3.3: UV mask design for the optimization of the exposure times during cassette development. The masks $a, b$ and $c$ are of the width $0.5 \mathrm{~mm}, 0.75 \mathrm{~mm}$ and $1 \mathrm{~mm}$ respectively.

Overall, 3 different channel widths of $1 \mathrm{~mm}, 0.75 \mathrm{~mm}, 0.5 \mathrm{~mm}$ and depths of $127 \mu \mathrm{m}$, $250 \mu \mathrm{m}, 500 \mu \mathrm{m}$ and $800 \mu \mathrm{m}$, obtained by using spacers made of silicone sheets of the specific thickness were used. Several monoliths were made with each combination of 
channel widths and depths. The monoliths were exposed to UV light for different time periods and the resulting channels were observed for good quality channels, both visually and by measuring the formed channel widths using a microscope.

\subsection{Results and Conclusion}

The Table 3.1 shows the summary of the optimized exposure time for various combinations of channel thickness and widths.

\begin{tabular}{|l|c|c|c|}
\hline $\begin{array}{l}\text { Channel } \\
\text { Thickness/Depths }\end{array}$ & $1 \mathrm{~mm}$ & $0.75 \mathrm{~mm}$ & $0.5 \mathrm{~mm}$ \\
\hline $127 \mu \mathrm{m}$ & $6 \mathrm{~s}$ & $6 \mathrm{~s}$ & $5 \mathrm{~s}$ \\
\hline $250 \mu \mathrm{m}$ & $8 \mathrm{~s}$ & $7 \mathrm{~s}$ & $7 \mathrm{~s}$ \\
\hline $500 \mu \mathrm{m}$ & $15 \mathrm{~s}$ & $12 \mathrm{~s}$ & $10 \mathrm{~s}$ \\
\hline $800 \mu \mathrm{m}$ & $22 \mathrm{~s}$ & $20 \mathrm{~s}$ & $12 \mathrm{~s}$ \\
\hline
\end{tabular}

Table 3.1: Exposure time summary

Summary of the optimized exposure times for different combinations of channel widths and thicknesses 
Initially, the width of the UV mask was measured using the scaled eye piece on the microscope. Once the channels were formed, they were also observed under the microscope to measure the actual width of the channel for each exposure time and both thicknesses were compared to determine any significant difference in widths. The exposure times for which the actual width of the channel was closest to the width of the UV mask were selected to be the optimized exposure times. They were also visually observed for the smoothness of the channel walls and the tapering of the widths if any.

The exposure times obtained were directly proportional to the thickness and the width of the desired microfluidic channel. Table 3.1 shows that the longest exposure time (22 seconds) is for the cassettes with the largest width and thickness ( $1 \mathrm{~mm}$ wide and 800 $\mu \mathrm{m}$ thick). While for channel dimensions of $127 \mu \mathrm{m}$ depth and $0.5 \mathrm{~mm}$ width, the exposure time was the shortest ( 5 seconds). These optimized exposure times were taken into consideration while developing the microfluidic cassettes of the desired dimensions. Since this optimization was done for just single straight channels, the exposure times for more complex designs might be slightly different from the values in the Table 3.1. If one does need more complex designs for the microfluidic channels, it is a good idea to optimize the exposure times separately for each design. 


\section{CHAPTER 4}

\section{METHOD VALIDATION}

Our main hypothesis for this research work is that (a) There is an increase in the value of the bulk capacitance of a given solution as the concentration of the bacteria in it increases; and (b) We can measure the increase in capacitance effectively using our electrical method. Hence, the initial work focused on demonstrating that our method was able to accurately estimate the capacitance of a solution, and that capacitance of a solution changed with an increase in the number of suspended bacteria to ensure that the hypothesis is acceptable and to validate our method. This was done by conducting 2 sets of experiments.

\subsection{Determining Dielectric Constant of Various Solutions}

Dielectric constant or relative permittivity of a medium is the ratio of the absolute permittivity of the medium $(\mathcal{E})$ to the permittivity of free space $\left(\varepsilon_{0}\right)$. Proteins are composed of amino acids and have well defined 3-D structures. An interesting feature of the protein solution is that the value of its dielectric constant is often found to be significantly larger than the value of pure water. The value of the dielectric constant of protein solution increases with increase in the concentration of the protein (Nandi and Bagchi 1998). As the protein concentration increases, the concentration of the bulk water decreases leading to a slower time scale of dielectric relaxation. This is because more 
bound water is present around the protein molecules as the protein concentration is increased and hence faster free water is replaced by slower bound water (Nandi and Bagchi 1998; Nandi et al. 2000). These changes lead to an increase in the dielectric constant of protein. Thus, as the concentration of protein solution increases, there is also a corresponding increase in the dielectric constant of the solution. On the other hand, for sugar solutions, such as sucrose, the dielectric constant decreases with increasing concentrations (Malmberg and Maryott 1950). This value of dielectric constant of various solutions can be determined by measuring the bulk capacitance of different concentrations of the solution. The bulk capacitance value is directly proportional to the dielectric constant of the solution, indicating that an increase in bulk capacitance value will lead to an increase in dielectric constant and a decrease in its value leads to a decrease in the dielectric constant value. The calculated values of dielectric constants can then be compared to the actual literature values to confirm our hypothesis.

To do this, we used five different samples - Bovine Serum Albumin (BSA), myoglobin, sucrose, AHA and Escherichia coli. Increasing concentrations of each of these samples were prepared and measured for its impedance using the impedance analyzer. The data obtained from the analyzer was then analyzed as mentioned in detail in Chapter 2 and the value of bulk capacitance was determined. The capacitance values obtained for different concentrations of the solutions were in turn used to identify dielectric constant $(\mathcal{E r})$ values for different concentrations of solutions. The values calculated by this method were verified with the theoretical Er values and those measured by others using the standard method (Nandi and Bagchi 1998) for different concentrations of solutions and results were correlated. 
The value of $\mathcal{E r}$ is calculated from the bulk capacitance value as:

$$
\varepsilon r=\mathrm{C}_{\mathrm{b}} / \mathrm{K}
$$

Where

\section{Er- Dielectric Constant}

$\mathrm{C}_{\mathrm{b}}$ - Bulk capacitance of the protein solution

K- Cell constant

$=\mathrm{C}_{\mathrm{bo}} / \varepsilon_{\mathrm{ro}}$

$$
\begin{aligned}
\text { Where } \mathrm{C}_{\mathrm{bo}} & =\text { Bulk capacitance of the solvent used } \\
\varepsilon_{\mathrm{ro}} & =\text { Dielectric constant of the solvent used }
\end{aligned}
$$

Thus, if we know the bulk capacitance value of the solution (obtained by our detection method) and the value of $\mathrm{K}$, we can calculate the value of dielectric constant for different solutions.

The biological cells consist of a plasma membrane and a cell wall with a cytoplasm and that can be depicted with a two-shell electric model. A typical E. coli cell consists of a highly conducting cytoplasm, an inner plasma membrane and a cell wall. The two-shell model is able to represent this with a conducting sphere (cytoplasm) covered with 2 concentric insulating thin shells of plasma membrane and cell wall. This model given by Asami (Asami 2002) takes into account the detailed morphological structure of a microbial cell. The complex permittivity of the cell as given by Asami (2002) is:

$\varepsilon_{c}^{*}=\varepsilon_{w}^{*} \frac{2 \varepsilon_{w}^{*}+\varepsilon_{p}^{*}-2 w\left(\varepsilon_{w}^{*}-\varepsilon_{p}^{*}\right)}{2 \varepsilon_{w}^{*}+\varepsilon_{p}^{*}+w\left(\varepsilon_{w}^{*}-\varepsilon_{p}^{*}\right)}$

where $\varepsilon_{\mathrm{w}}{ }^{*}$ is the complex permittivity of the cell wall, $\varepsilon_{\mathrm{p}}{ }^{*}$ is given by:

$\varepsilon_{p}^{*}=\varepsilon_{m}^{*} \frac{2 \varepsilon_{m}^{*}+\varepsilon_{i}^{*}-2 v\left(\varepsilon_{m}^{*}-\varepsilon_{i}^{*}\right)}{2 \varepsilon_{m}^{*}+\varepsilon_{i}^{*}+v\left(\varepsilon_{m}^{*}-\varepsilon_{i}^{*}\right)}$ 
$v=(1-d / R)^{3}$,

$\mathrm{R}$ is the outer radius of the shell-sphere

$\mathrm{d}$ is the thickness of the shell

$\mathrm{w}=\left(1-\mathrm{d}_{\mathrm{w}} / \mathrm{R}_{\mathrm{c}}\right), \mathrm{d}_{\mathrm{w}}$ is the wall thickness and $\mathrm{R}_{\mathrm{c}}$ is the outer cell radius.

$\varepsilon_{\mathrm{m}}=$ Dielectric constant of medium/solvent used

$\varepsilon_{\mathrm{i}}=$ Dielectric constant at the electrode interface

The predicted relative permittivity of the bacterial cells is given by:

$\varepsilon_{1}=\frac{9 * 9 * \varepsilon_{m} * R * w * P h i}{\left(d *\left((2+w) *(2+p h i)+\left(2 *(1-w) *(1-p h i) *\left(\frac{K_{w}}{K_{a}}\right)\right)^{2}\right.\right.}$

Phi is the volume fraction of the bacterial cells in suspension

$\mathrm{K}_{\mathrm{w}}$ and $\mathrm{K}_{\mathrm{a}}$ are the wall and medium conductivity, respectively.

The total suspension (containing bacterial cells with media) dielectric constant is given by the sum of the medium dielectric constant and predicted relative permittivity of the bacterial cells.

\subsection{Sample Preparation}

Bovine Serum Albumin (BSA) (Fisher Scientific), myoglobin (Sigma®), sucrose (Fisher Scientific), AHA (Sigma®), Escherichia coli K12 (ATCC 23716), were used in this study. In order to obtain different concentrations of each of the samples - BSA, myoglobin, sucrose and AHA, a stock solution was prepared and then appropriately diluted in 0.05X Phosphate Buffer Saline (PBS). The concentrations obtained for 
myoglobin and BSA were 80,100, 120, 140,160, $180 \mathrm{mg} / \mathrm{ml}$, Sucrose was 10, 20, 30, 40, 50, $60 \mathrm{wt} \%$ and AHA was $0.2,0.4,0.6,0.8,1,1.5,2,3 \mathrm{M}$.

Bacteria E. coli was serially diluted in bacterial nutrient media Tryptic Soy Broth (TSB) and milk for this study. Both TSB, and milk which were used as media for serial dilutions and the cassettes in which the measurements were to be made were autoclaved at $121^{\circ} \mathrm{C}$ and $15 \mathrm{psi}$ to ensure that there was no contamination by bacteria. The bacteria was grown in TSB overnight at $37^{\circ} \mathrm{C}$ to obtain a log culture, which was assumed to have a concentration of $10^{8} \mathrm{CFU} / \mathrm{ml}$. Two samples each of $1 \mathrm{ml}$ of this overnight culture, were spun down using a centrifuge and the supernatant discarded. The 2 pellets (bacteria) each were then resuspended in TSB and milk and serially diluted in them to obtain concentrations of bacteria - 10, 100, 1000, $10^{4}, 10^{5}, 10^{6}, 10^{7} \mathrm{CFU} / \mathrm{ml}$.

\subsection{Impedance Measurements}

Once all of the samples were prepared, a small volume $(\sim 250 \mu 1)$ of each of the samples was taken out, injected into the cassettes and impedance measurements were made at room temperature using the Agilent 4294A impedance analyzer (Agilent Technologies, CA, USA) over a frequency range of $1 \mathrm{kHz}$ to $100 \mathrm{MHz}$. The data

obtained from the impedance analyzer was then analyzed using the software $\mathrm{ZView}^{\mathrm{TM}}$ to obtain the bulk capacitance values. These values were then used to determine the dielectric constant of the sample as described in section 4.1. 


\subsection{Results}

The data generated by the impedance analyzer consists of 3 parameters: frequency (f) over which the sample was scanned (1 kHz to $100 \mathrm{MHz})$ and the corresponding resistance $(\mathrm{R})$ and reactance $(\mathrm{X})$ values. Once the data was obtained for each of the sample sets, it was analyzed using the software $\mathrm{ZView}^{\mathrm{TM}}$, the details of which has already been explained in Chapter 2. In short the analysis was done by feeding the equivalent electrical circuit to the software, and fitting the data from the analyzer to the circuit. The analysis gives values for each of the individual parameters in the circuit which includes the bulk capacitance values. The bulk capacitance values obtained were plotted against the concentration of the sample. 


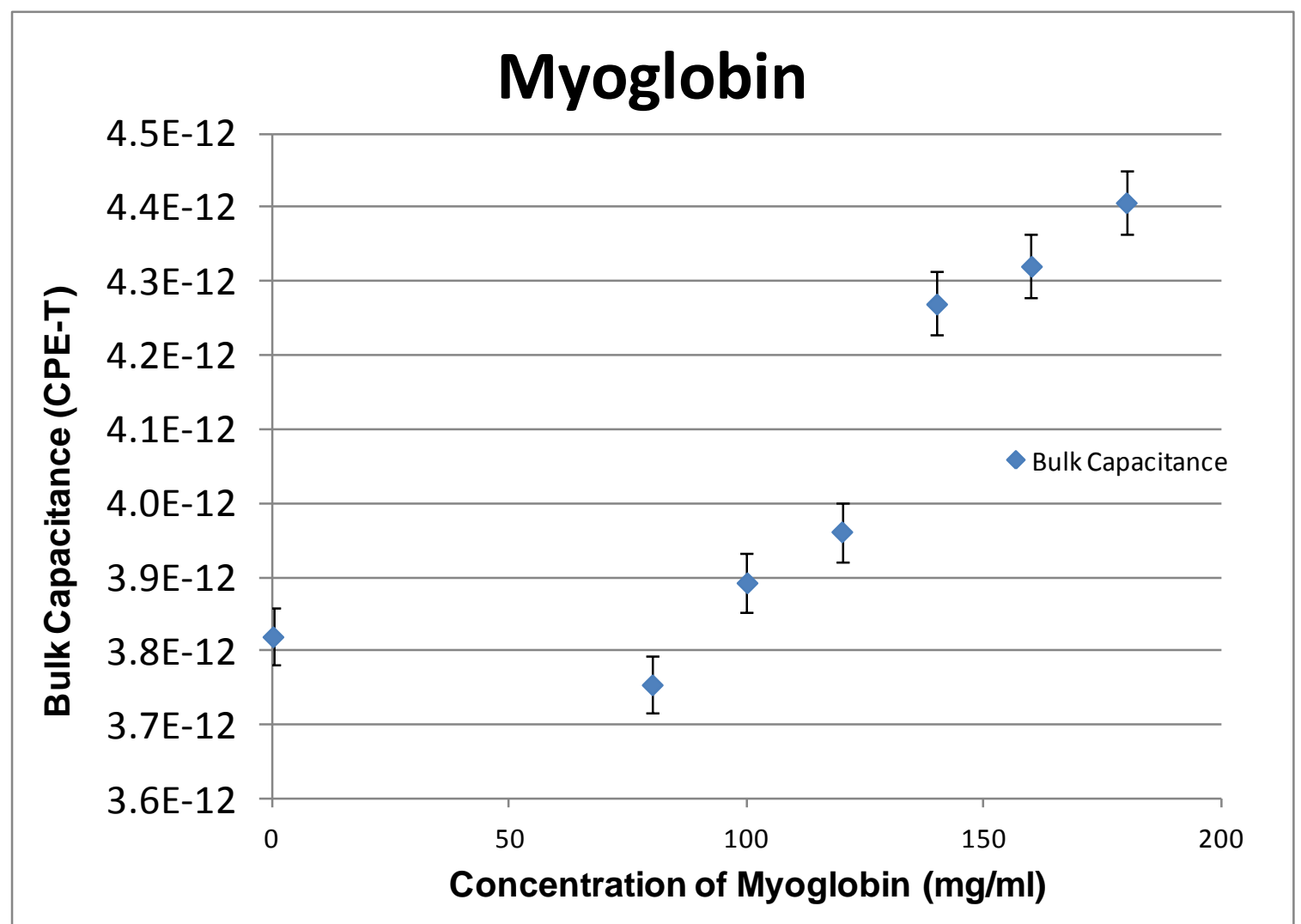

Figure 4.1: Myoglobin plot

Gives the plot the of the bulk capacitance values obtained using ZView ${ }^{T M}$ over concentration of myoglobin ranging from $80-180 \mathrm{mg} / \mathrm{ml}$. 

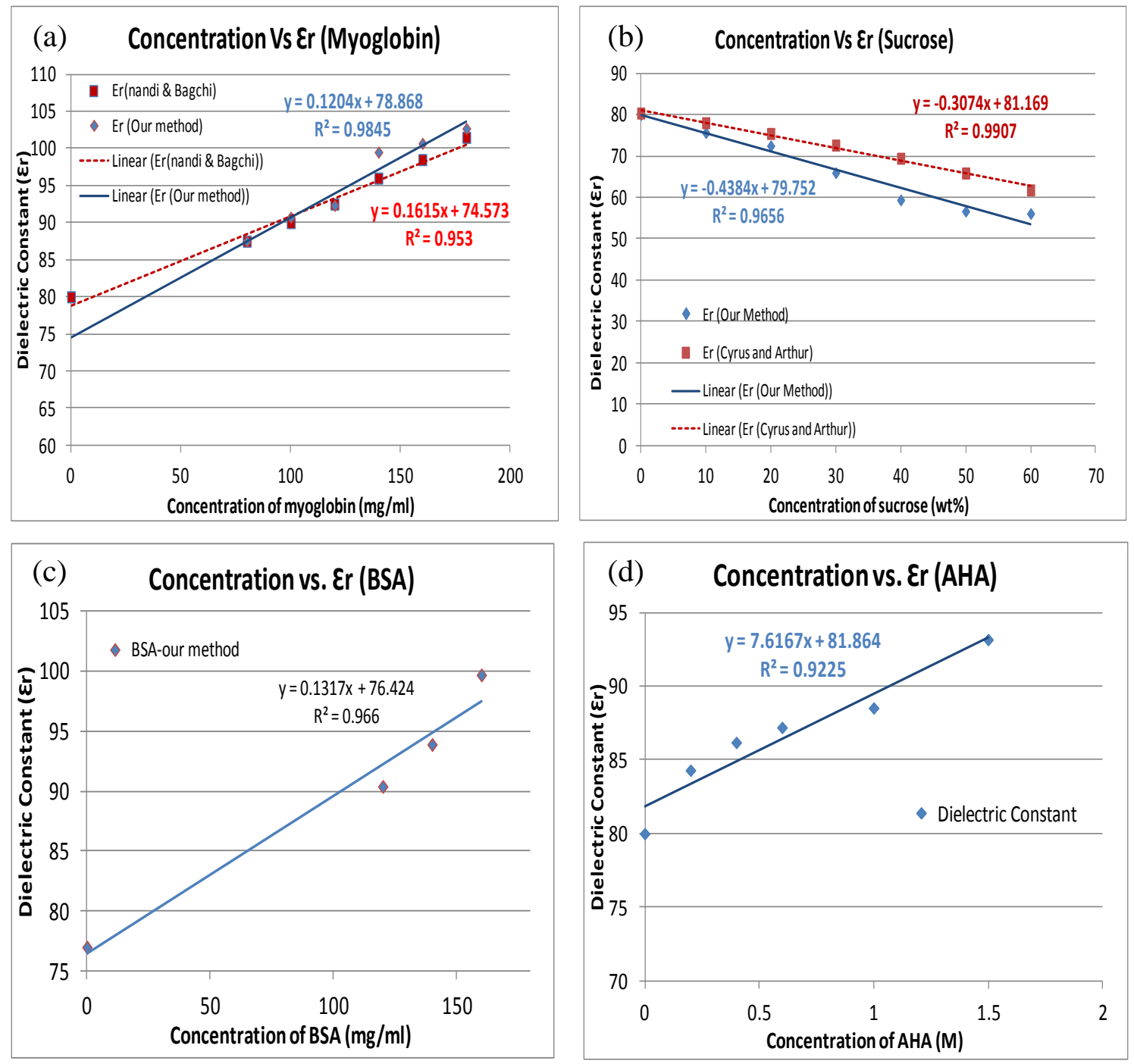

Figure 4.2: Dielectric constant plots for myoglobin(a), sucrose(b), BSA(c) and AHA(d)

Dielectric constant over different concentrations of Myoglobin (a), Sucrose (b), BSA (c) and AHA (d). The Blue diamonds here indicate values of dielectric constants calculated using our method, while the red squares give the corresponding dielectric constant obtained by literature review. 
Figure 4.1 gives a plot of bulk capacitance values over different concentrations of myoglobin. Here, it can be seen that there is an increase in the value of bulk capacitance with an increase in the concentration of myoglobin solution. To validate our results further, we calculated the dielectric constant corresponding to each of the bulk capacitance values obtained, the calculations of which is explained in detail in Section 4.1. Figure 4.2 shows the comparison of the $E r$ values obtained using our method (represented by diamonds) and Er values from literature (represented by squares) for myoglobin (Figure 4.2(a)), Sucrose (Figure 4.2(b)), BSA (Figure 4.2(c)) and AHA (Figure 4.2(d)). It can be seen that there is a good correlation between the literature values (represented by red squares) and the observed dielectric constant values (represented by blue diamonds) for myoglobin and sucrose obtained using our method, indicating that our method of detection is capable of tracking the changes in bulk capacitance values for different solutions and that the $C_{b}$ values obtained can be used as a reliable indicator of the actual bulk capacitance value of the suspension. 

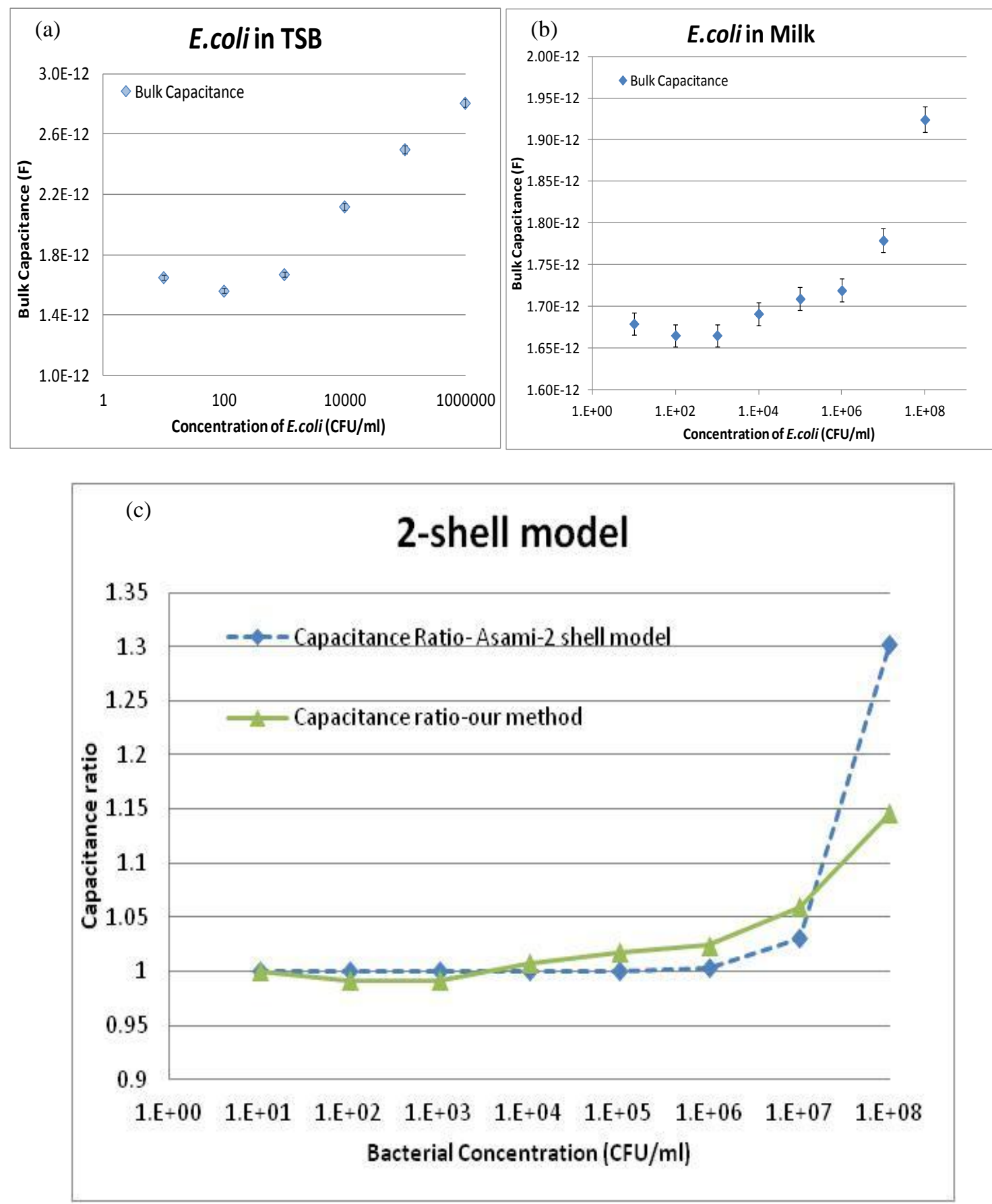

Figure 4.3: Bulk Capacitance plots of E.coli in TSB (a), Milk (b) and 2-shell model fitting for E.coli in Milk (c) 
The results of serial dilution studies with E.coli in TSB, milk and 2-shell model fitting of the capacitance ratios of E.coli in Milk are shown in Figure 4.3. Figure 4.3(a) and (b) gives the bulk capacitance values (represented by blue diamonds) over different concentrations of bacteria. Each bulk capacitance value is associated with a error bar which represents the error percentage of the fit of the data to the circuit model using the software $\mathrm{ZView}^{\mathrm{TM}}$. From the figure it can be seen that, at low concentrations of bacteria (typically below $1000 \mathrm{CFU} / \mathrm{ml}$ ), we did not observe any significant change in the value of the bulk capacitance of the bacterial suspension (as seen due to the overlap of the error bars with respect to the zero concentration reading). However, at and over $1000 \mathrm{CFU} / \mathrm{ml}$, the error bar on the bulk capacitance values no longer overlaps with that of the initial reading indicating that beyond that value, the bulk capacitance value is significantly different from the lowest concentration. Thus, our method of detection is able to detect the proliferating bacteria, once the concentration of the bacteria in the sample reaches $\sim 10^{3}-10^{4} \mathrm{CFU} / \mathrm{ml}$. Thus, even if one starts with a very low initial concentration of bacteria in a suspension with growth media, once the bacteria is incubated to grow, it will only take a few (6-10) doubling times for the bacteria to be detected using our method. Figure 4.3(c) represents the fitting of a 2-shell bacterial model to the capacitance ratios obtained using our method experimentally. The values of the capacitance ratios obtained by fitting the model faintly/qualitatively follow the actual values. A better fitting model taking into consideration the bacterial cell as a 3-shell spheroid model consisting of outer membrane, periplasmic membrane and inner membrane can be developed which might help achieve closer values of the fit with respect to the actual experimental results. 


\title{
CHAPTER 5
}

\author{
RAPID DETECTION OF BACTERIAL PROLIFERATION \\ IN FOOD SAMPLES USING MICROCHANNEL \\ IMPEDANCE MEASUREMENTS AT MULTIPLE \\ FREQUENCIES
}

The material presented in this chapter was reviewed and published as "Rapid Detection of Bacterial Proliferation in Food Samples Using Microchannel Impedance Measurements at Multiple Frequencies"

Sachidevi Puttaswamy ${ }^{1}$ and Shramik Sengupta ${ }^{1}$

Sensing and Instrumentation for Food Quality and Safety, 2010, 4:108-118.

\subsection{Abstract}

We present a novel method for detecting viable bacteria in suspensions such as milk and apple juice. Underlying the technique is the fact that bacteria in aqueous suspensions can store a large amount of charge, and thus, act like (non-ideal) capacitors. Thus, increased numbers of bacteria due to proliferation increases the capacitance of the bulk of the suspension. However, this increase cannot be directly measured since the capacitance of the solid-liquid interface ("double layer") in effect "screens" the latter. We present a method (derived from an earlier one) that is able to discern such changes with 
high sensitivity and robustness. We also demonstrate its ability to monitor food quality/safety by detecting bacterial proliferation in "real world" liquid food samples like milk and apple juice. We are able to detect $\sim 1,10,100$, and $1000 \mathrm{CFU} / \mathrm{ml}$ of E. coli in milk in about $4.5,3,2$, and 0.5 hours, respectively. For the same initial loads, the corresponding times to detection (TTDs) for Lactobacillus in apple juice are

approximately 8, 6, 4, and 1 hour(s). These represent a greater than 4-fold reduction in TTD when compared to automated systems on the market such as RABIT, Bactometer etc. We can achieve such low TTDs for low initial loads since, due to the much greater effective charge holding capacity of bacterial cells (compared to surrounding media), we are able to detect a change in the overall bulk capacitance of the suspension as the bacterial numbers cross a threshold of around $500 \mathrm{CFU} / \mathrm{ml}$.

\subsection{Introduction}

Some bacteria may survive procedures like pasteurization designed to eliminate them in various liquid food products like milk (Juffs and Deeth 2007), juices (Goverd et al. 1979), etc., or may be inadvertently introduced during further processing (Drake et al. 2002; Kirov et al. 1993). These bacteria typically cause spoilage, leading to estimated economic losses of $\$ 1$ billion each year (Gould and Russell 2003). The surviving bacteria, when pathogenic may even cause outbreaks of food borne illnesses among consumers who assumed that the product was risk-free since it had been pasteurized ( Centers for Disease Control and Prevention (CDC). 2009; Dalton et al. 1997; Goh et al. 2003). In the USA alone, 76 million food borne illnesses occur per year leading to 5000 
deaths (Mead et al. 2000) and is estimated to have an economic impact of \$6.5-\$34.9 billion each year (Buzby and Roberts 1997).

Detecting and quantifying bacteria that survive treatments such as pasteurization is an important step in assuring food quality and safety, and in meeting standards set by appropriate governing bodies or trade organizations. For instance, the US Pasteurized Milk Ordinance requires "Grade A" pasteurized milk to have a total bacterial count of $\leq$ 20,000 CFU/ml and a coliform count of $\leq 10 \mathrm{CFU} / \mathrm{ml}$ (Hayes and Boor). As a consequence, those who produce/market the food have to perform microbiological tests to satisfy themselves and the governing bodies, regarding the efficacy of their processes designed to keep numbers of bacteria within the stipulated range. It is important to their economic operation that they do so with the least possible expenditure of resources (material and labor).

There are presently several ways to detect bacteria in liquid samples like milk and juice. They can be broadly classified into three broad classes: (a) traditional methods such as plate cultures and biochemical assays, (b) DNA and antibody based methods, often involving micro/nano particles and fluorescence, (c) other "automated" techniques that rely on monitoring the effects of bacterial metabolism on the medium. Of these, traditional methods are the most extensively used, and often serve as the standard to which other techniques are compared. However, they are tedious, labor intensive and have very long times to detection ranging from overnight to weeks depending on the type of the organism and medium used. DNA and antibody based methods overcome many of the disadvantages of the traditional methods. They are rapid, require less reagents and labor, and are able to identify the species/strain of the bacteria present relatively easily. 
However, they are not able to distinguish between viable and dead bacteria, and hence their applicability in many situations (such as that described earlier) is limited.

There has been a lot of interest in developing methods that are automated (to reduce labor) and miniaturized (to reduce material costs) for bacterial detection in samples. There are a large number of automated methods already commercialized. They include devices such as the Bactec ${ }^{\mathrm{TM}}$ that detects the amount of radio-labeled carbon dioxide released, Coli-Check ${ }^{\mathrm{TM}}$ swabs that use Bromocresol Purple as an indicator to measure the decrease in $\mathrm{pH}$ due to bacterial metabolism, and the Bactometer ${ }^{\mathrm{TM}}$ (Bactomatic Ltd.), Malthus $2000^{\mathrm{TM}}$ (Malthus Instruments Ltd.) and RABIT ${ }^{\mathrm{TM}}$ (Don Whitley Scientific Ltd.) systems, that use electrical impedance. A summary of various automated methods already commercialized, and the times to detection (TTD) for these methods (for various mentioned initial loads) are given in Table 5.1. 


\begin{tabular}{|l|l|l|l|l|}
\hline Commercial name & Method employed & Initial load & Microorganisms & TTD \\
\hline RABIT (Don Whitley & Change in solution & $1 \mathrm{CFU} / \mathrm{ml}$ & coliforms & $16.1 \mathrm{hrs}$ \\
UK) (Madden and & conductance & & & \\
Gilmour 1995b; & & & & \\
Pridmore and Silley & & & & \\
2001) & Impedance & $>10^{5} \mathrm{CFU} / \mathrm{ml}$ & Mainly E. coli & 4 hours \\
\hline Bactometer (Bio & & & \\
Merieux, Nuertingen, \\
Germany) (Zafari and \\
Martin 1977)
\end{tabular}

Table 5.1: Commercial automated systems

Comparison of some of the commercially available automated systems for bacteria detection. 
The common underlying feature of these techniques, including those which use electrical impedance, is that they rely on bacterial metabolism to produce a discernible change in a material property of the medium (such as $\mathrm{pH}$, optical density, amount of carbon dioxide dissolved, electrical conductivity). The amount of metabolite processed by an individual bacterium is extremely small. [Based on our knowledge that the specific oxygen consumption rate for $E$. coli is $20 \mathrm{mmol}$ of oxygen per hour per gram (dry weight) of bacteria (Andersen and Von Meyenburg 1980b) and a typical bacterium has a dry weight $\sim 10^{-12} \mathrm{~g}$ (Borsheim et al. 1990b), we estimate that one bacterium consumes only $2 \times 10^{-14}$ moles of oxygen in one hour]. Hence, there has to be a sufficiently large number of bacteria present (either a priori or arising due to proliferation from the smaller number initially present) before the signal generated (change in the material property of the suspension) can be effectively measured. If the bacterial count in the original suspension happens to be small (1000 CFU/ml or lower), one must wait for cells to proliferate to an appropriately high number (often $\sim 10^{6} \mathrm{CFU} / \mathrm{ml}$ or greater) before a discernable change in the physical properties of the medium (such as $\mathrm{pH}, \mathrm{O}_{2} / \mathrm{CO}_{2}$ concentration, conductivity, etc.) can be noticed. Thus, for low initial loads, current commercial automated systems take almost as long as the plate-cultures (overnight or longer) to provide the desired result.

In the recent past, there have been efforts to increase the ease of handling, cut costs, and most importantly, reduce times to detection (TTD) by using microfluidic systems to miniaturize the automated methods. Bashir and coworkers have developed chip-based micro-devices in which $\mathrm{pH}$ and impedance are monitored in order to detect bacterial metabolism(Gomez et al. 2002; Yang et al. 2005), and various additional 
modifications like the use of interdigitated microelectrodes (Yang et al. 2004a; Yang et al. 2004b), and arrays of microelectrode based biosensors (Radke and Alocilja 2004, 2005) have been tried in order to increase the sensitivity of measurements (with respect to conventional electrodes), and thus, further decrease the TTD. While these efforts were successful (in the sense that their TTDs are lower than those of the commercially available devices), they continue to be limited by the amount of time it takes for bacterial metabolism to significantly alter the composition of the medium when bacterial loads are low. Bashir and co-workers attempted to overcome this drawback by concentrating the bacterial cells from dilute samples to a small volume by using dielectrophoresis (DEP) prior to culture, and then detecting changes in medium composition as before (GomezSjoberg et al. 2005). While the culture time needed for detection was reduced, one needs to take into account the time needed for concentration using DEP (an additional 2-3 hours) as well to get effective TTDs. Again, while successful, the actual method of detection still relies on bacterial metabolism, with its inherent limitations (as discussed earlier).

In our previous work (Sengupta et al. 2006b), we proposed a different approach to detect the presence of viable bacteria in a given suspension. We argued that since bacteria can store a lot of charge ( 100 X more than an equal volume of water) (Poortinga et al. 1999b), an increase in the number of bacteria through proliferation would lead to a measureable increase in the charge storing capacity (capacitance) of the medium, and hence the reactance (out of phase component of the impedance) between two metal electrodes in contact with the medium. Other previous researchers (Felice and Valentinuzzi 1999) had also had the same idea, but they had failed to detect any such 
change in reactance. We showed, using mathematical models, that this was because, given the range of $\mathrm{AC}$ signal frequencies they used $(<1 \mathrm{MHz})$ and the geometry of the system, the electrochemical interface between the electrodes and the aqueous solution (the "double layer") effectively "screened" the bulk solutions. Our models also led us to propose that we could detect increased capacitance of the bulk medium due to bacterial proliferation by (a) changing the geometry of the system (using narrow and long, capillary-like geometries as depicted in Figure 5.1) to decrease the inverse-RC time constant of the system, and (b) measuring the reactance of the system at multiple frequencies, and tracking the frequency at which a reactance minima was recorded. We showed, using our proposed method and reactance (impedance) measurements up to 1 $\mathrm{MHz}$, our ability to detect an initial load of $\sim 100 \mathrm{CFU} / \mathrm{ml}$ E. coli in Tryptic Soy Broth (TSB) in about 3 hours.

Here, we report two advances. Firstly, we enhance the sensitivity of our electrical technique through the use of (a) high frequency impedance measurements up to $100 \mathrm{MHz}$ (b) a refined electrical model for our system that is better able to describe the behavior of the bacterial particles at these high frequencies, and (c) more rigorous data analysis. Secondly, we demonstrate that the technique is suitable for monitoring food quality/safety by detecting bacterial proliferation in "real world" liquid food samples like milk and apple juice. 


\subsection{Materials and Methods}

\subsubsection{Sample preparation and inoculation of bacteria into samples}

Escherichia coli K12 (ATCC 23716), and Lactobacillus acidophilus (Nature's Life $^{\mathrm{TM}}$ Apple-honey Lactobacillus acidophilus probiotic) were used in this study. In order to obtain load cultures, E. coli K12 was incubated overnight at $37^{\circ} \mathrm{C}$ in Tryptic Soy Broth (TSB) (Bacto $\left.{ }^{\mathrm{TM}}, \mathrm{BD}\right)$, Lactobacillus acidophilus was incubated at $30^{\circ} \mathrm{C}$ for about $48 \mathrm{hrs}$ in MRS Broth (Difco ${ }^{\mathrm{TM}}, \mathrm{BD}$ ). These were then used, in appropriate dilutions, to seed the samples in which we monitored bacterial proliferation using our method. These samples included those of TSB into which we loaded E. coli (to compare our new technique to our previous work), and two representative liquids to study the ability of the method to detect bacteria in food samples, [2\% reduced fat milk (Prairie Farms ${ }^{\mathrm{TM}}$ ) for $E$. coli and preservative free Apple juice (Florida's Natural $^{\mathrm{TM}}$ ) for Lactobacillus acidophilus].

To facilitate growth of lactobacilli in the apple juice, its $\mathrm{pH}$ was adjusted to about 6 by adding potassium hydroxide (about $1 \mathrm{ml}$ of $10 \mathrm{M} \mathrm{KOH}$ to $50 \mathrm{ml}$ of apple juice). The media and the food samples were all autoclaved at $121^{\circ} \mathrm{C}$ at $15 \mathrm{psi}$ to ensure no presence of live bacteria in them. This ensured the right concentration of the bacteria in the sample when we artificially inoculated it with our bacteria of interest. The samples were allowed to cool down to room temperature before bacterial inoculation. The bacterial suspension, after being incubated for specified time periods, was initially assumed to contain approximately $10^{9} \mathrm{CFU} / \mathrm{ml}$ bacteria. One millimeter of E. coli $K 12$ and $1 \mathrm{ml}$ of Lactobacillus acidophilus were taken in separate eppendorf tubes and centrifuged for 8 
minutes to settle the bacteria down as a pellet. Then the supernatant was discarded and pellets were resuspended in an equal volume of food samples in which they were to be detected. Then the suspension was serially diluted and inoculated into the liquid samples to have different initial concentrations of bacteria in them and also simultaneously the samples were plated onto petri-dishes to get the actual initial concentration of the inoculated bacteria in each sample.

\section{$\underline{5.3 .2}$ Experimental Design}

Four sets of $9 \mathrm{ml}$ of each of the liquid samples (TSB, milk or apple juice) were added to the incubating tubes. Each tube was inoculated with the bacteria to be detected such that the final concentrations of the bacteria in the tubes were approximately 1,10 , 100 and $1000 \mathrm{CFU} / \mathrm{ml}$, respectively. The tubes were then allowed to incubate for a time period of 8 hours for the 1, 10, $100 \mathrm{CFU} / \mathrm{ml}$ concentrations and 5 hours for the $1000 \mathrm{CFU} / \mathrm{ml}$ concentration. At regular time intervals $(30 \mathrm{~min}$ for $1000 \mathrm{CFU} / \mathrm{ml}$ and 1 hour for $1,10,100 \mathrm{CFU} / \mathrm{ml})$ small volume $(\sim 250 \mu \mathrm{l})$ of the sample was taken out, injected into the cassettes and impedance measurements were made using the Agilent 4294A impedance analyzer (Agilent technologies, CA, USA) over the frequency range of $1 \mathrm{kHz}$ to $100 \mathrm{MHz}$. Simultaneously at every time interval, $100 \mu \mathrm{l}$ of the sample was taken, diluted appropriately and plated onto petri-dishes to give the actual concentration of bacteria at that hour in the sample. The whole process was repeated independently at least 3 times for each targeted initial load of the system $(1,10,100$ or $1000 \mathrm{CFU} / \mathrm{ml})$ and for all liquids (TSB, milk and apple juice). 
The microfluidic cassettes used for the measurement was fabricated using liquid phase photo-polymerization of a commercially available UV curable polymer (Loctite $363^{\mathrm{TM}}$ ), a process that has been described elsewhere (Sengupta et al. 2004b) in detail. The final device is shown in Figure 1(a) inset. The cassettes were sterilized in an autoclave at $121^{\circ} \mathrm{C}$ before use. After each of the experiments, the electrical connectors were replaced; cassettes were washed thoroughly with soap, bleach, alcohol and water, and then autoclaved.

\subsubsection{Electrical Measurement Technique}

When the cassettes are loaded with an aliquot from the sample being investigated, the suspension fills the micro-channels along which electrodes are positioned as shown in Figure 5.1(a). An Agilent 4294A Impedance Analyzer was used to measure the electrical impedance between the electrodes at multiple (>500) frequencies between $1 \mathrm{kHz}$ to 100 $\mathrm{MHz}$. The instrument measures the magnitude and phase of the AC current that flows through the suspension upon the application of a sinusoidal AC voltage of $500 \mathrm{mV}$ (peakto-peak), and calculates the Impedance (resistance and reactance) from the measurements. Since the current is not in-phase with the applied sinusoidal voltage, the Impedance, which can be considered as the AC analog of the DC resistance, has both an in-phase component called the resistance (R), and an out-of-phase component called the reactance $(\mathrm{X})$. It is typically represented as a complex number and as shown in Equation 5.1: 


$$
\mathrm{Z}=\mathrm{R}+j \mathrm{X}
$$

where $j=\sqrt{-1}$

Alternatively, the impedance can also be represented completely by its magnitude $(|\mathrm{Z}|)$ and its phase angle $\theta$. The magnitude and phase angle, respectively, of the impedance, are related to the resistance and reactance by the following equations:

$$
\begin{aligned}
& \mathrm{Z}=\sqrt{\left(R^{2}+X^{2}\right)} \\
& \theta=\operatorname{Tan}^{-1}\left(\frac{X}{R}\right) \ldots
\end{aligned}
$$

The impedance analyzer measures impedance by measuring the resistance (R) and reactance $(\mathrm{X})$ for each sample, over the frequency range of $1 \mathrm{kHz}$ to $100 \mathrm{MHz}$ and thus generates the data set containing the values of $\mathrm{R}$ and $\mathrm{X}$ at multiple ( $>500$ ) frequencies.

\subsubsection{Circuit Model and Data analysis}

In our previous work (Sengupta et al. 2006b), we had proposed that a system consisting of an aqueous solution in a microfluidic channel with electrodes on either end (Figure 5.1(a)), can be represented by an equivalent electrical circuit as shown in Figure 5.1(b). As seen, this takes into account the existence of electrochemical "double layers" on the surfaces of charged metal electrodes. Hence, the net measured impedance ( $\left.\mathrm{Z}_{\text {measured }}\right)$ is, as shown by the accompanying equation, affected by not only by the presence of conductive and capacitive (charge-storing) elements in the bulk, but also by such elements present at the electrode-solution interface. In fact, the interface capacitance 
is typically more than 1000 times greater than the bulk capacitance, as a result of which previous investigators (Felice and Valentinuzzi 1999) were not successful in measuring any changes in the latter, although it was expected that since bacteria can store charge (Poortinga et al. 1999b), their proliferation would lead to an increase in bulk capacitance. In our previous work, we showed how the use of micro-channel geometry serves to concentrate the electrical field and raise the effective bulk resistance of the suspension, thereby allowing us to pick up signatures of increased bulk capacitance. Specifically, the signature that we looked at was a shift in the plot of measured reactance $(\mathrm{X}) \mathrm{v} / \mathrm{s}$ frequency $(\omega)$ in the range of $1 \mathrm{kHz}$ to $1 \mathrm{MHz}$.

Since the effects of the electrochemical double layer are less pronounced at high frequencies (as can be seen from the equation accompanying Figure 5.1(b)), we had initially assumed that obtaining readings at higher frequencies (up to $100 \mathrm{MHz}$ ) would enable us to obtain more sensitive readings using the same metric (shift in $\mathrm{X} v / \mathrm{s} \omega$ curves). However, our experiments (results not shown) were unsatisfactory. Due to the increased contribution to the measured reactance from the bulk resistance at these higher frequencies, even extremely small changes in the value of this quantity (that we attribute to thermal fluctuations in our lab) caused shifts in the $\mathrm{X} \mathrm{v/s} \omega$ curve. Thus, our efforts to increase the sensitivity of our old measurement technique led to false positives. We hence needed a way to distinguish the true positives (shift due to an increase in bulk capacitance) from false positives (shift due to other reasons such as an increase in the bulk resistance). 
(a)

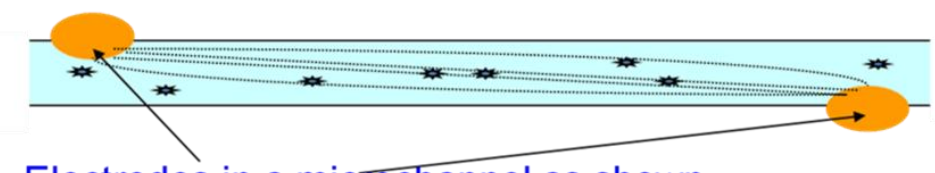

Electrodes in a microchannel as shown

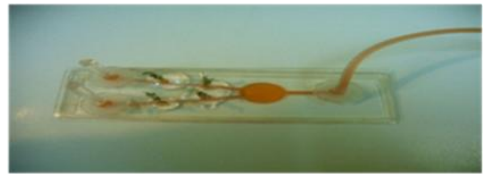

(b)
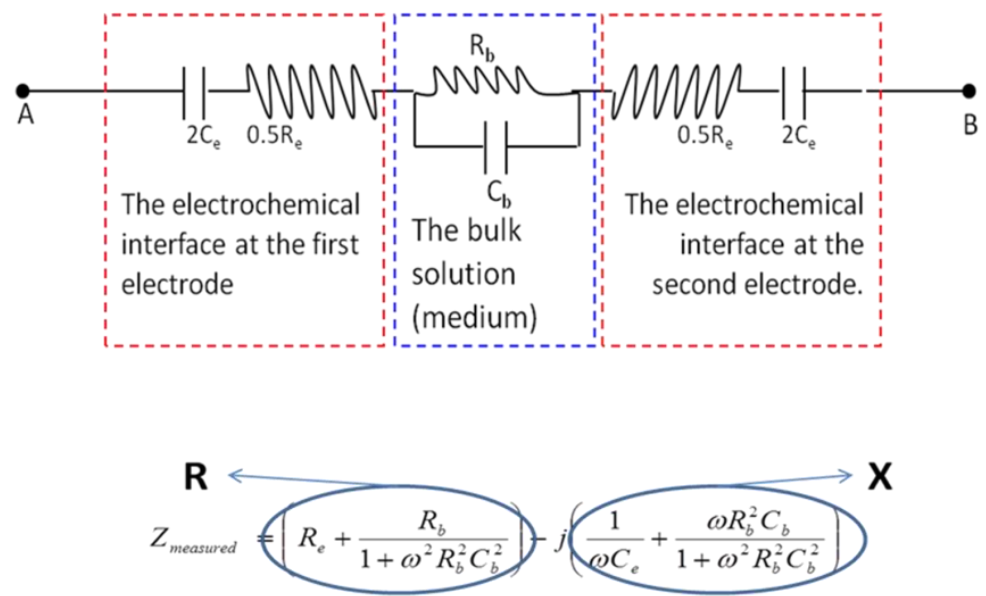

Figure 5.1: Schematic and electrical representation of microchannel with equation

(a) Schematic representation of the micro-channel with electrodes on either end loaded with suspension harboring bacteria (actual image in inset). The spatial confinement of the electrical lines of force ensure sensitivity of electrical measurements to the bacteria present $(b)$ Equivalent circuit representation of the channel with suspension of bacteria and electrodes [Re - Resistance at the electrode-suspension interface, Ce-Capacitance at the electrode-suspension interface, $R b$ - bulk resistance, $\mathrm{Cb}$ - bulk capacitance]

To overcome this hurdle, we chose to adopt a different procedure, viz. to evaluate changes in all individual components of the circuit. To do so, we took the values of resistance $(\mathrm{R})$ and reactance $(\mathrm{X})$ measured by the impedance analyzer and fit that to our 
equivalent circuit model using a commercial circuit analysis software $\left(\mathrm{ZView}^{\mathrm{TM}}\right)$. This software, when given the circuit model and data set, is be able to estimate each of the individual parameters $\left(\mathrm{R}_{\mathrm{e}}, \mathrm{C}_{\mathrm{e}}, \mathrm{R}_{\mathrm{b}}\right.$ and $\left.\mathrm{C}_{\mathrm{b}}\right)$ of the circuit. Using this technique, it was further seen that while the circuit model used earlier Figure 5.1(b) works well at low frequencies, it does not hold very well at higher frequencies. Replacing the bulk capacitance $\left(\mathrm{C}_{\mathrm{b}}\right)$ with a Constant Phase Element (CPE) in the circuit (yielding the circuit shown in Figure 5.2(a), provided a much better fit to the data obtained - as shown in Figure 5.2(b). 


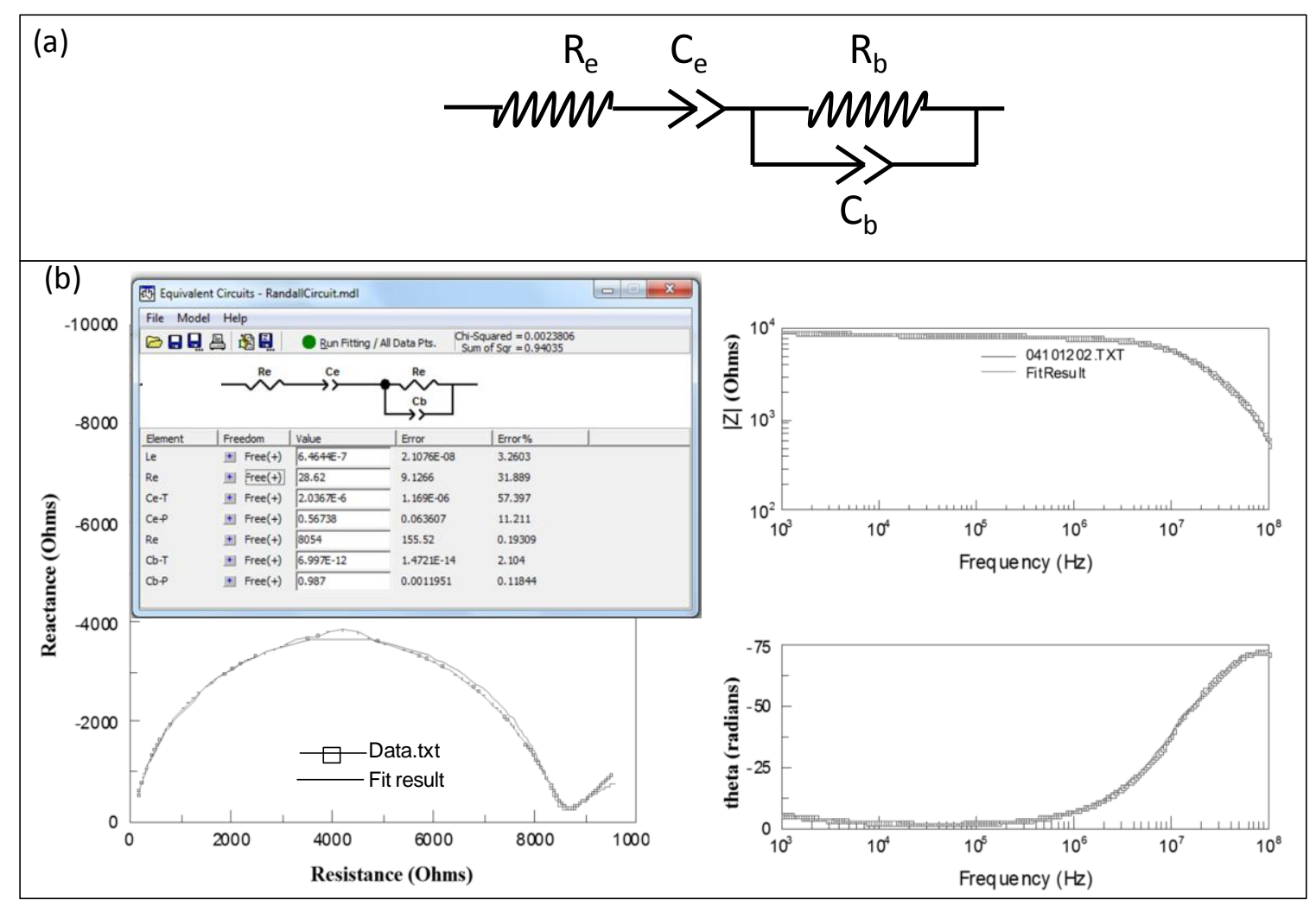

Figure 5.2: ZView $^{T M}$ analysis

(a) Equivalent circuit diagram as used in the ZView ${ }^{T M}$ software with a constant phase element used to represent the charge-holding behavior of the bulk suspension (bacteria), instead of an ideal capacitor (as in Figure 5.1) (b) Results obtained using Z-view ${ }^{T M}$, when the data from Impedance Analyzer (red line) was fitted to the circuit model with the parameters on the right (green line). 
The CPE is a non-intuitive circuit element that replaces a capacitor in a circuit when there is some type of non-homogeneity in the system, delaying or impeding the movement of charge carriers ( Barsoukov and MacDonald 2005). In more mathematical terms, the impedance of a CPE is given by the equation:

$$
Z=0-j\left(\frac{1}{(\omega Q)^{n}}\right)
$$

Where $\omega=2 \Pi f$

As shown in Equation 5.3, the impedance of the CPE is defined by two values: the magnitude component CPE-T (Q) measured in $\mathrm{Fs}^{\mathrm{n}-1}$ and the phase component CPE-P (n). If CPE-P (n) equals 1 then the equation is identical to that of a capacitor. Since it intuitively seems likely that bacteria, while able to store charge, may not behave like ideal capacitors, compensating its non-ideality with CPE was observed to show better fit. We could identify a need for using a CPE for our data analysis, as the arc of the ColeCole plot for our impedance data was a depressed semicircle or an arc of the circle rather than a perfect semicircle as would be the case if the bacteria behaved like ideal capacitors (Meland 2007). The phenomenon of depressed semicircles has been given multiple explanations including surface roughness, varying thickness or composition of coatings, non-uniform current distributions. In short, the main reason is due to some nonhomogenous nature or distribution of value of some physical property of the system. In Barsoukov and MacDonald 2005, they point out that although it is difficult to isolate the exact CPE behavior, it fits the experimental data well enough that it can provide a useful modeling element even if the true nature of the system in unknown. 
The value of the CPE-P (n) is not a constant but is different for different samples. When doing the analysis using the $\mathrm{ZView}^{\mathrm{TM}}$ software, initially the data is loaded to the software and the circuit to which it had to be fit is constructed. Then, each of the circuit parameters $\left(\mathrm{R}_{\mathrm{e}}, \mathrm{C}_{\mathrm{e}}, \mathrm{R}_{\mathrm{b}}, \mathrm{CPE}-\mathrm{T}\right.$, and CPE-P) are provided with initial guess values and the system numerically optimizes the values to obtain the best fit for the system as a whole over the range of frequencies examined. Convergence is often not guaranteed, and sometimes the system converges to a solution that is "obviously" wrong - as seen from the visual fit of the computed circuit model impedance curves (green lines in Figure 5.2(b)) to the actual data (red lines in Figure 5.2(b)). Since the charge-holding behavior of individual bacteria cells presumably do not change with an increase in their number, in some (but not all) cases, the value of the CPE-P is fixed to the value obtained earlier for the same sample to help the software reach an apparently correct numerical solution. In the other cases, it is seen that the software itself arrives at a set of values for the circuit parameters in which the value of the CPE-P is extremely similar (if not identical) to that obtained previously for the same sample. It may be noted, though, that the values of CPE$\mathrm{P}$ that we obtained appear to be a function of the bacterium studied and the medium used with values of approximately $0.96,0.97$, and 0.986 for E.coli in TSB, E. coli in Milk, and L. acidophilus in apple juice respectively. We do not have, at present, any basis for predicting a-priori what the value of the CPE-P will be for a particular system. Presumably, they may even differ among different samples of the same product (e.g. different batches of milk, depending on the protein and fat content). 


\subsubsection{Establishing Times to Detection (TTDs) for Individual Samples}

The CPE-T value generated by the software provides a measure of the chargestoring capability of the suspension being investigated. Over a period of time, this quantity is expected to increase with an increase in the number of bacteria, and one can conclusively state that there are viable bacteria in the sample when one observes this quantity (CPE-T) increasing significantly. The time needed to make this observation (significant increase in the value of CPE-T) is the Time to Detection (TTD) for our system. As illustrated in Figure 5.3, the confidence interval (error bar) of the CPE-T value obtained from the 1-hour impedance readings overlaps with that of the corresponding 0-hour (initial) value. In contrast, the confidence interval of the CPE-T value obtained at the 2-hour impedance measurement does not overlap with that of the initial reading. Thus, it is possible to state conclusively that the charge holding capacity of the suspension increased (presumably due to proliferation of bacteria) after 2 hours, but not after 1 hour. Hence our TTD for this case is 2 hours. It may be noted that the values of CPE-T closely track the plate counts (red squares in Figure 5.3) of bacteria in the system at that particular time (obtained from aliquots harvested at that time). Also, had we taken a reading at 1.5 hours; it is possible we could have detected a significant change in CPE-T earlier, but since we did not do so, we can only report a TTD of 2 hours. 


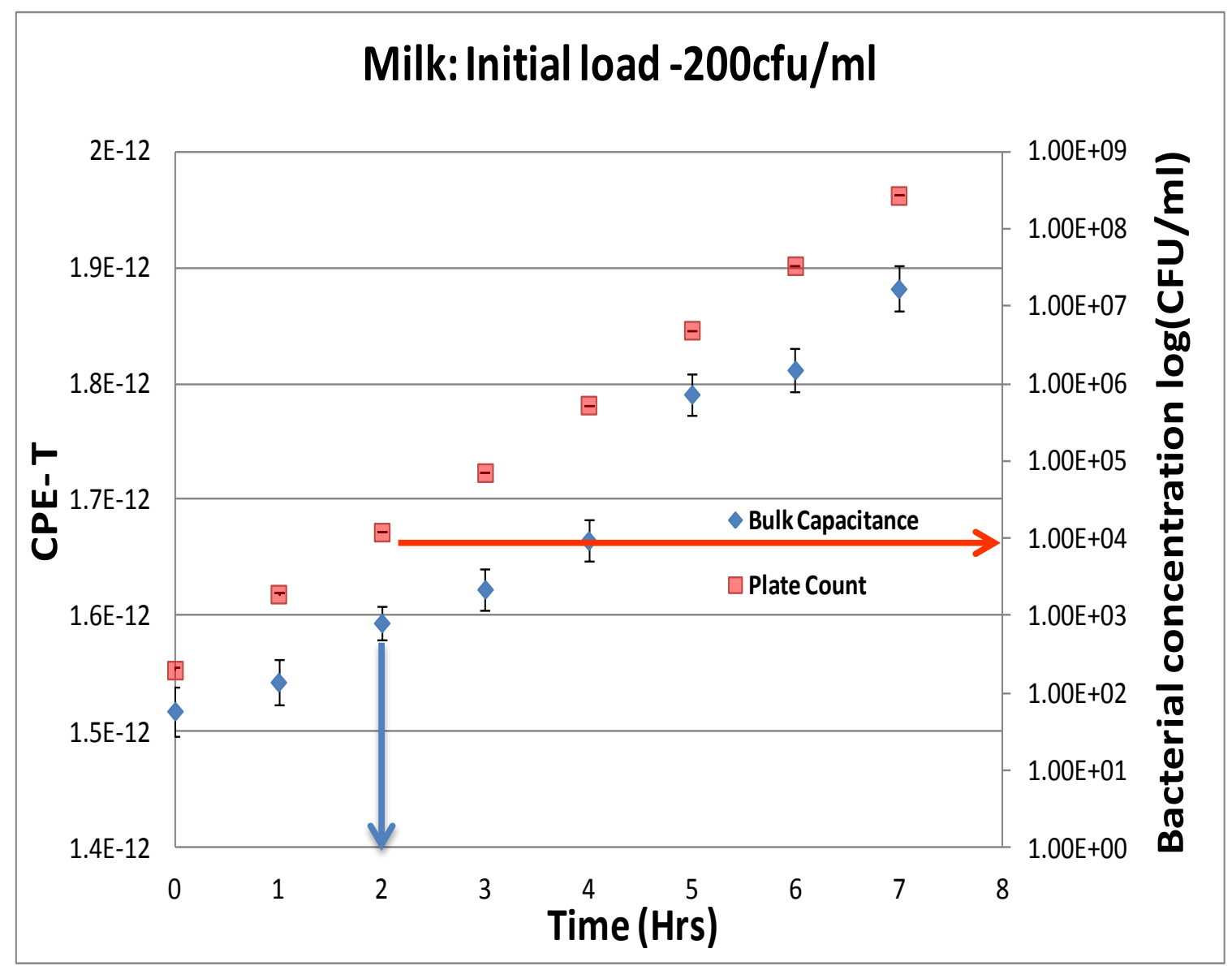

Figure 5.3: Time to detection plot for milk with E.coli

Figure 5.3: Plot showing the increase in the bulk capacitance (Blue diamonds) with actual increase in the concentration of the bacteria (red squares) in the suspension. The plot also indicates the time to detection (shown by the arrow), For this sample, the error bar of CPE-T value of 2 hours does not overlap with the error bar of zero-hour reading and hence 2 hours is considered as the time to detection. 


\subsection{RESULTS}

\subsubsection{Ability of the Calculated CPE-T Value to Track True Bacterial Counts}

The validity of our method using CPE-T (obtained as described earlier) as an indicator of bacterial load in the system is illustrated in Figure 5.4. As seen in this figure, the CPE-T values closely track the actual bacterial numbers present (as obtained using plate counts) irrespective of whether the bacterial numbers hold steady (as occurs in the lag and saturation/stationary phase), rise, or decline. The decrease in CPE-T values as bacteria die off seems to indicate that dead bacteria are not as capable as live ones of storing charge. We speculate that this is due to the active transport ion pumps no longer being functional for these dead bioparticles and membrane integrity being disrupted which prevents any charge storage (Melkikh and Seleznev 2007). 


\section{E coli in TSB}

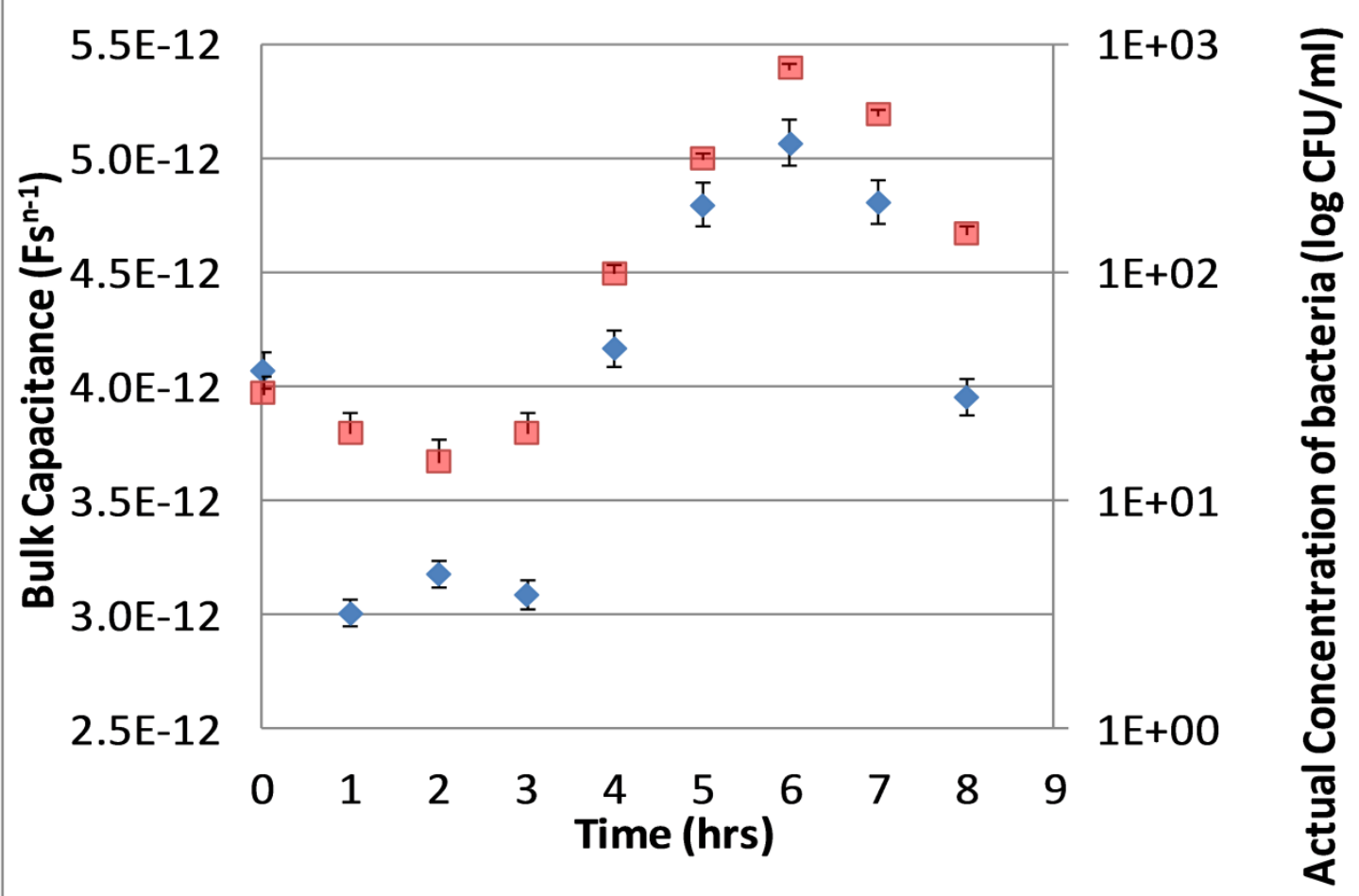

\section{Bulk Capacitance $\square$ Actual concentration of E.coli}

Figure 5.4: Bulk Capacitance vs. concentration plot for E.coli in TSB

Plot of CPE-T values (blue diamonds) and actual concentration of bacteria in the sample (red squares) at various points in time for a system consisting of E. coli suspended in Tryptic Soy Broth (TSB). The data show the ability of our method to closely track the true bacterial count. 


\subsubsection{Times to Detection (TTD) as a Function of Initial Bacterial Loads}

Three types of samples (TSB, milk and apple juice) were inoculated with 4 different initial bacterial loads (targeted to be 1,10,100,1000 CFU/ml), impedance measurements were taken at specific intervals (half hour or one hour), and the impedance data were analyzed using $\mathrm{ZView}^{\mathrm{TM}}$ software to obtain the CPE-T values as described in the previous section. These values were used to obtain TTDs using the criteria explained using Figure 5.3. Some more of such typical plots of CPE-T Vs time which gives the TTDs for each sample are shown in Figure 5.5, with the arrows indicating the TTD for that sample with respective initial bacterial load.

In a few cases, mostly for L. acidophilus in apple juice, a significant lag phase is observed. During this period, bacteria do not grow, and sometimes even die - as indicated by the plate count data (e.g red squares of Figure 5.4). In such cases, a better estimate of the capabilities of our system is obtained by subtracting the lag phase time ( 2 hours in our example) when we calculate our TTD for the given initial load in the given system. For example, in the case shown in Figure 5.4, although the significant increase in CPE-T from the initial value is detected only at the fourth hour, the TTD of the system is taken to be 2 hours since for the first two hours, the bacteria in the suspension were in the lag phase.

Each experiment with the targeted initial load of bacteria in a specific sample ws repeated three times to ascertain the reproducibility of the method. A more accurate estimate of the true value of the initial loads could only be obtained the next day, once plate counts were obtained. Hence, we have twelve points each for TSB, milk and apple juice (some of these points overlap very closely, and are not distinguishable). These 
points are used to calculate a line of bet fit using linear regression, and these lines are also shown in Figure 5.6. The equations for these lines provide the best estimate of the time that our system will take to detect a given load of a particular type of bacterium in a particular substrate.

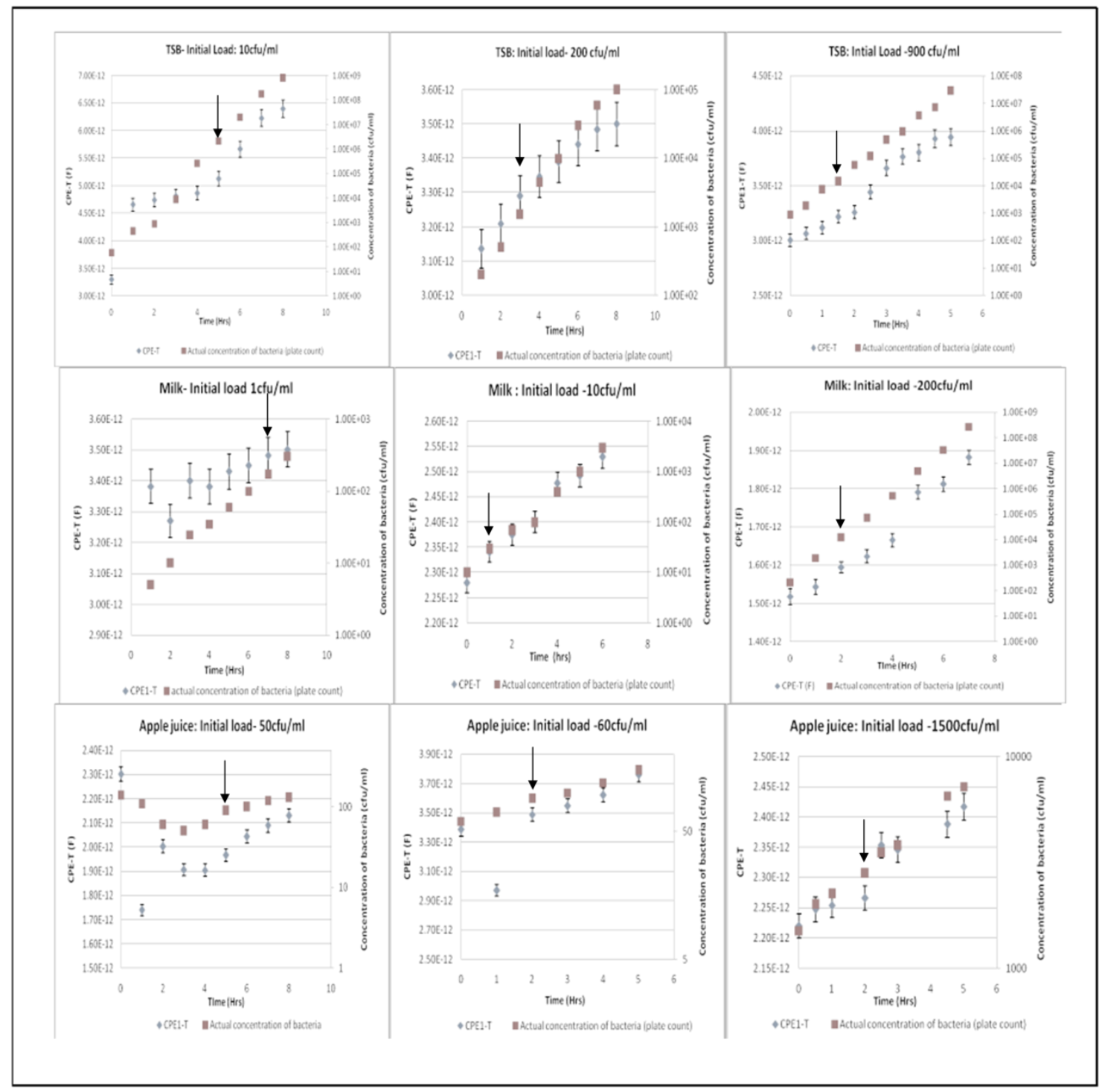

Figure 5.5: Bulk capacitance plots for E.coli in TSB, milk and apple juice $C P E-T \mathrm{v} / \mathrm{s}$ time plot for some representative samples with different initial bacterial loads. The "Time to Detection" (TTD) for each is indicated by an arrow. 

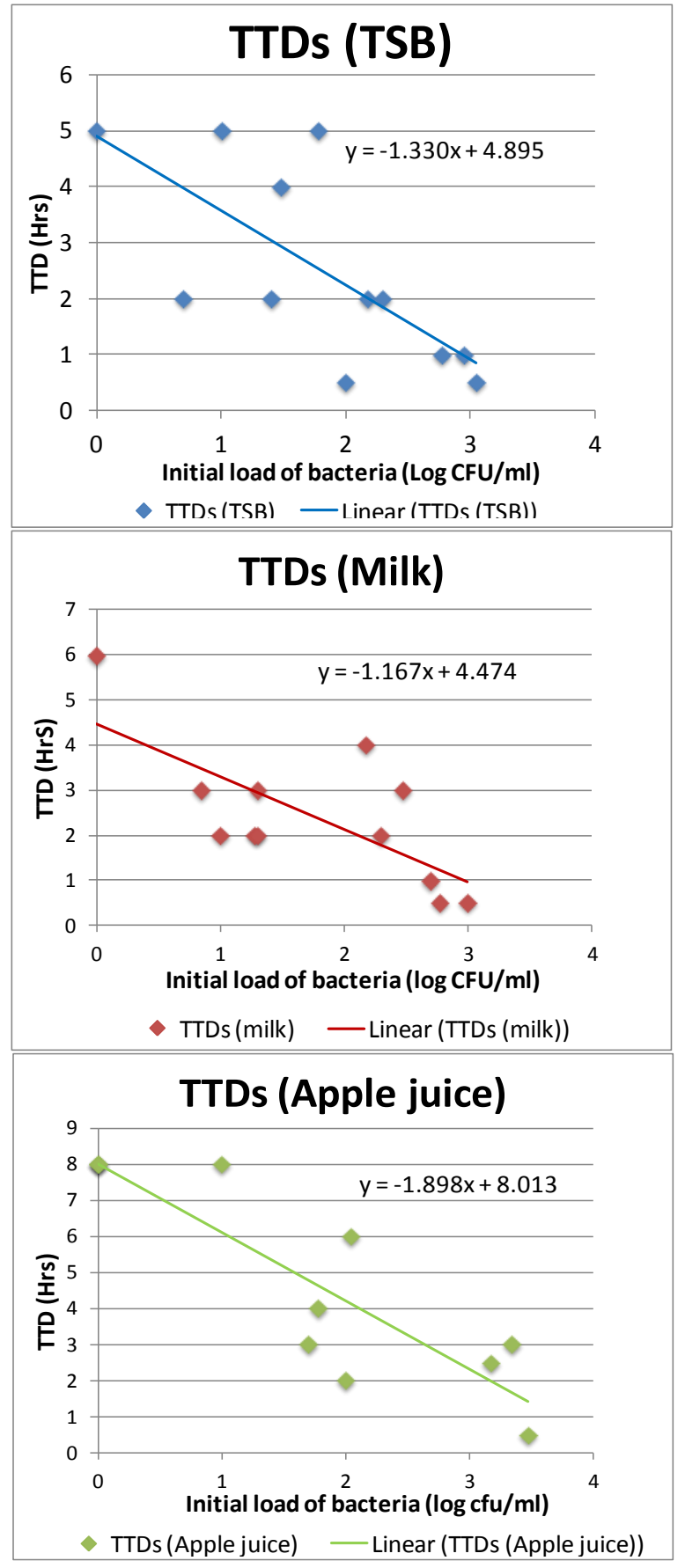

Figure 5.6: TTD vs. Initial load plots for TSB, Milk and Apple Juice

Consolidated plots showing the variation of the Time to Detection (TTD) as a function of the initial bacterial load for multiple experiments with E. coli in Tryptic Soy Broth (left), E. coli in milk (center), and Lactobacillus in apple juice (right) 


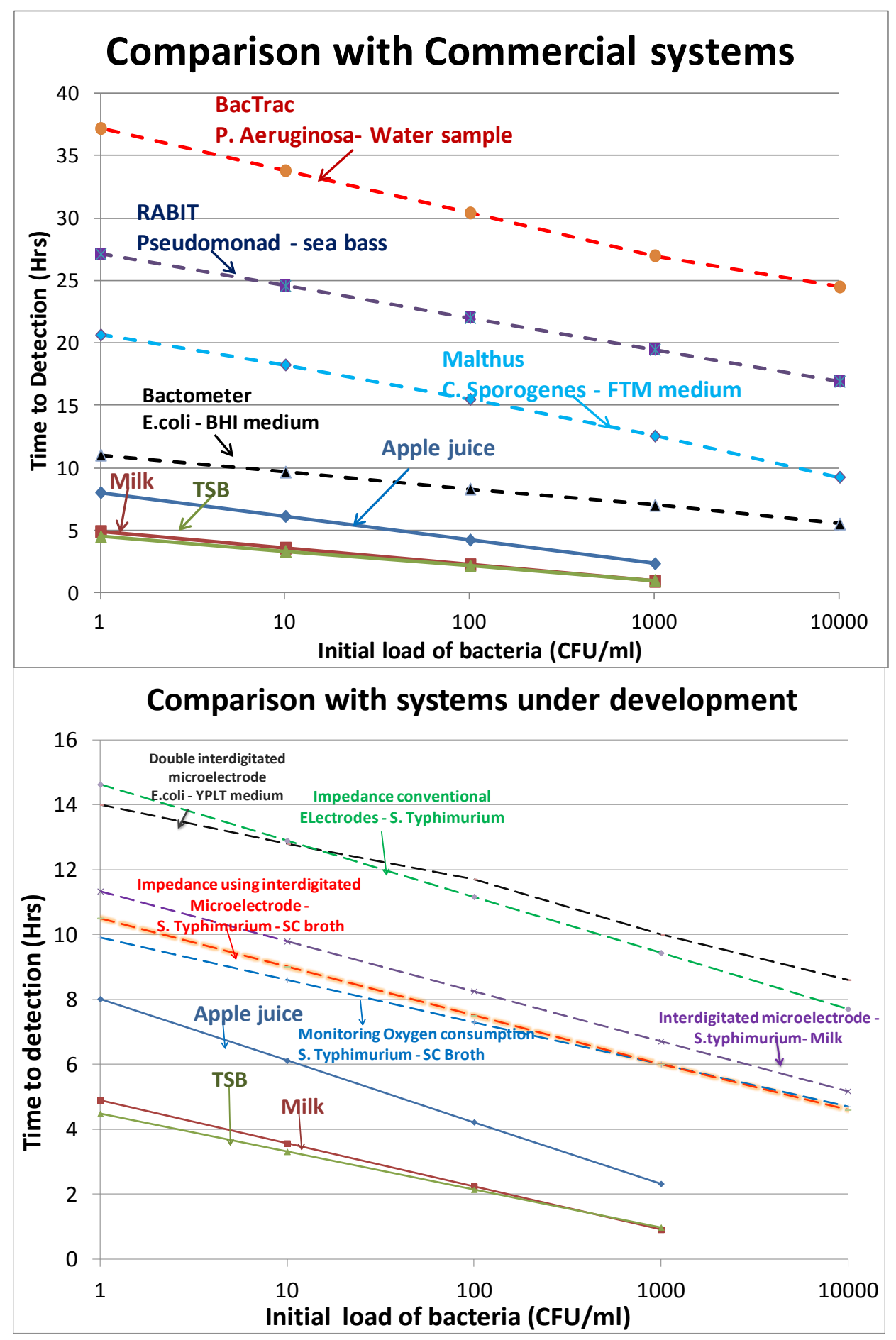

Figure 5.7: Comparison plots of TTD vs. Initial load for different systems

Comparing TTDs obtained using our system (solid lines) to (a) those of the commercial systems already on the market, and (b) other, especially microfluidic, systems under development (dashed lines). 


\subsection{Discussion}

As seen in Figure 5.6, there is an inverse relationship between the (log) initial load of bacteria in a sample, and the TTD of proliferating bacteria using our method. In this it is similar to methods that rely on detecting the effects of bacterial metabolism such as Bactometer $^{\mathrm{TM}}$, $\mathrm{RABIT}^{\mathrm{TM}}$, etc. This is expected since the presence of more bacteria (our method) leads increased metabolite consumption/generation. Also, as in the case of RABIT $^{\mathrm{TM}}$, Bactometer ${ }^{\mathrm{TM}}$ etc., one can generate a calibration plot for a particular type of suspension, and the TTD can be used to estimate the initial load of the system. The scatter that we observe seems qualitatively comparable to the data used to generate calibration curves for RABIT etc (Koutsoumanis et al. 2002). The scatter arises due to multiple reasons. Two, that we believe contribute the most in our case, are uncertainties in the estimates of the initial loads, and differences in metabolic state of members within and between populations seeded. In other words, although we plot our TTDs against a "known" initial load based on plate counts, this "known" value itself is subject to some degree of uncertainty - typically of the order of the square root of the true number of particles present (Poisson distribution) (Rosner 2006). Thus, if the suspension being incubated had $100 \mathrm{CFU} / \mathrm{ml}$ of bacteria (true value) we expect that a $100 \mu \mathrm{l}$ sample we introduce into our cassette, or use for plating, will have 10 CFUs. However, there is also a $33 \%$ chance that the sample we actually isolate will have either less than $7(10-\sqrt{ } 10)$, or greater than $13(10+\sqrt{ } 10)$ bacteria. In addition, a certain fraction of the bacterial cells that constitute the inoculum may remain in the lag phase slightly longer than others. This may not be readily captured by the plate counts taken to determine the initial load (since in plates, they get adequate time to grow). Since we are operating at low concentrations 
(low numbers of bioparticles), these sampling uncertainties are liable to introduce a greater relative error. Despite these sources of error, our TTD data still show a clear trend in the manner expected (inverse with respect to log initial load).

Another characteristic of our method is that the TTD is a function of the doubling time of the proliferating bacteria. The faster that a given bacterium doubles, the shorter is our TTD. In the present study, we worked with two different bacteria: E. coli $K-12$ that has a doubling time of 27 minutes at $37^{\circ} \mathrm{C}$ (Skarstad et al. 1985), and Lactobacillus acidophilus that has a doubling time of 50-60 minutes at $30^{\circ} \mathrm{C}$ (Coyette and Shockman 1973). Thus the doubling time of lactobacillus is about 2 times that of E. coli. The TTDs for lactobacillus are also correspondingly longer ( 8 hours for $1 \mathrm{CFU} / \mathrm{ml}$ and 4.5 hours at $100 \mathrm{CFU} / \mathrm{ml} \mathrm{v/s} 4.5$ hours and 2 hours, respectively, for E. coli at the same initial loads). For initial loads of $1000 \mathrm{CFU} / \mathrm{ml}$ or higher, we were able to detect proliferation in half an hour (the shortest time interval we used) for E. coli (and in one case, for lactobacillus as well). Thus, at these relatively higher loads, we were able to detect the bacteria within one cycle of division. For lower initial bacterial loads, at the points in time where significant changes in CPE-T values are detected, their concentration in the sample (as estimated from the plate counts) is typically between 200-1000 CFU/ml. As a rough ballpark estimate, we can claim that we are able to catch the bacteria in the act of doubling their numbers when there are about 500 of them present per $\mathrm{ml}$ of suspension. Since the volume of liquid between our electrodes is $\sim 20 \mu 1$, this implies that we are actually observing about 10 of these bacteria (reproducing to form 20) at that time.

As shown in Figures 5.7(a) and 5.7(b), our TTDs compare very well with automated techniques already on the market and other automated techniques in development. Figure 
5.7(a) gives the comparison of our detection method with that of the commercial automated systems- RABIT ${ }^{\mathrm{TM}}$ (Koutsoumanis et al. 2002), Malthus $2000^{\mathrm{TM}}$ (Dromigny et al. 1997), Bactometer ${ }^{\mathrm{TM}}$ (Buckland et al. 1983), BacTrac ${ }^{\mathrm{TM}}$ (Szita et al. 2007) currently on the market. Figure 5.7(b) gives the comparison between our system and some of the systems currently under development. Virtually all of these systems under development continue to rely on detecting the effects of bacterial metabolism on the medium properties, such as changes in $\mathrm{pH}$ (Yang et al. 2005), Conductivity (Grossi et al. 2008; Yang et al. 2005), oxygen concentration (Ruan et al. 2002), for detection. Some employ features and capabilities available through microfluidic systems, such as microinterdigitated electrodes (Varshney and Li 2008; Yang et al. 2004b) or preconcentration using dielectrophoresis (Gomez-Sjoberg et al. 2005; Yang and Bashir 2007) to try and reduce the overall TTDs. While they achieve low TTDs (3-9 hrs) for very high initial concentrations of bacteria ( 10,000 CFU/ml), they continue to have high TTDs (10-14 hours) at a low initial concentration of bacteria $(1 \mathrm{CFU} / \mathrm{ml})$. Thus, for any given initial load, our system is able to detect bacteria at least 3 to $4 \mathrm{X}$ faster than the competition. 


\subsection{Conclusion}

The method presented here represents a different approach to detecting viable bacteria than taken by virtually all other automated systems, including those currently under development. Instead of relying on monitoring the effects of bacterial metabolism, it monitors the charge carrying capacity of the suspension (which increases with increasing numbers of suspended particles capable of storing charge), and thus, directly tracks bacterial numbers. We have been able to use this method to detect low loads of bacteria in milk and apple juice much more quickly than current automated methods. This coupled with the fact that handling requirements are very similar to those used for current methods and that the reported method appears to be robust to minor fluctuations in temperature, encourages us to believe that the latter can be widely adopted for monitoring food quality and safety. Further, it can, in the future, also be applied to other areas where rapid detection of low bacterial loads is important, such as for environmental and clinical applications. 


\section{CHAPTER 6}

\section{NOVEL ELECTRICAL METHOD FOR EARLY \\ DETECTION OF VIABLE BACTERIA IN BLOOD \\ CULTURES}

The material presented in this chapter was reviewed and published as "Novel Electrical Method for Early Detection of Viable Bacteria in Blood Cultures" Sachidevi Puttaswamy, Byung-Doo Lee, Shramik Sengupta

Journal of clinical microbiology 2011, 49:2286-2289

\subsection{Abstract}

We present a novel electrical method for detecting the presence of viable bacteria in blood, which can provide results 4-10 times faster than current Automated Blood Culture Systems like the BACTEC ${ }^{\mathrm{TM}}$, BacT/Alert $^{\mathrm{TM}}$ etc.

Our method infers the presence of viable bacteria in suspensions by measuring the charge stored in the suspension's interior (the "Bulk Capacitance"). Suspended live bacteria store charge, thereby acting like dispersed capacitors and contributing to the Bulk Capacitance. Bacterial proliferation during culture causes the Bulk Capacitance to increase from its initial value. This increase serves as a "signature" indicating the presence of viable bacteria. The Bulk Capacitance (as distinct from the much larger "Interfacial Capacitance"), is measured by introducing aliquots drawn 
from the Blood Culture Broth into a microfluidic channel, obtaining Electrical Impedance $(Z)$ measurements at multiple frequencies $(\omega)$ from $1 \mathrm{kHz}$ to $100 \mathrm{MHz}$, and using the $\mathrm{Z}$ vs. $\omega$ data to calculate the value of the Bulk Capacitance.

Here, we report the performance of this technique on "synthetic" Blood Cultures (Blood, inoculated with known amounts of E. coli, added to Bactec Ped-Plus ${ }^{\mathrm{TM}}$ medium). We show that our method can discern a change in the Bulk Capacitance of the Blood Culture medium when bacterial concentrations reach $\sim 1000-10,000$ CFU/ml. With these low "Threshold Concentrations", we are able to detect $\sim 1, \sim 10, \sim 100$, and $\sim 1000 \mathrm{CFU} / \mathrm{ml}$ of $E$. coli in blood in 4, 3, 2, and 1 hour, respectively. For BACTEC $^{\mathrm{TM}}$, the corresponding Times to Positivity were approximately 16, 12, 10, and 8 hours, respectively.

\subsection{Introduction}

Bloodstream Infections (Septicemia) and the inflammatory response they induce (Sepsis) are among the leading causes of death. Each year in the US, there occur over 751,000 cases of severe Sepsis, out of which over 383,000 ( 51\%) require intensive care, and over 215,000 ( 29\%) are fatal (Angus 2001). More disturbingly, the mortality rates for patients with severe Sepsis have remained essentially unchanged (at around 30\%) over the last three decades (Dombrovskiy et al. 2007; Friedman et al. 1998). Part of the reason for its high mortality rate, is that Sepsis is difficult to diagnose. It starts out as a set of non-specific symptoms known as Systemic Inflammatory Response Syndrome (SIRS). SIRS is characterized by two or more of the following: hypothermia or fever, fast heart 
and/or respiratory rate, and a WBC count of $<4000$, or $>12,000$ cells $/ \mathrm{mm}^{3}$. When faced with a patient exhibiting SIRS, clinicians typically begin administration of broad spectrum antibiotics, and initiate the process to (a) confirm the existence of the infection, and (b) identify the infective organism. Typically, the first is achieved using a blood culture and requires $12-72$ hours to obtain a positive result (and 120 hours for samples to be deemed negative). Because of the high mortality rate of Sepsis and the non-specific nature of symptoms, blood cultures are often conducted to "rule out" the possibility of Sepsis. It has been reported that of the approximately 5 Million blood cultures performed in the US each year, only about 750,000 (15\%) turn up positive (Manning 2006). Once the presence of bacteria has been confirmed by a positive blood culture, the bacteria present can be identified in an additional 3-24 hours using a variety of methods ranging from traditional biochemical tests (such as those employed in API Strips ${ }^{\mathrm{TM}}$ ) to more "modern" methods such as those employing Polymerase Chain Reaction (PCR) based DNA analysis, those employing Fatty Acid Methyl Ester (FAME) analysis, etc. (Mancini et al. 2010). Only after the infecting bacteria have been identified, can clinicians commence with "targeted" antibiotic therapy (administration of antibiotics known to be effective against the infectious organism present). The long time interval in starting targeted antibiotic therapy (12-72 hours for culture, plus 3-24 hours for bacterial identification) is an important factor contributing to the high mortality rate of Sepsis. It has been estimated (Kumar et al. 2006) that for every hour of delay in starting targeted antibiotic therapy, the risk of death for a given patient increases by 6-1\%. Since the blood culture step is by far the longer of the two diagnostic steps needed, cutting down the Times to Positivity (TTPs) of blood cultures is a worthwhile goal. 
It remains worthwhile to try to reduce TTPs of blood cultures despite advances in molecular (primarily DNA-based) diagnostics that aim to determine the identity of bacteria directly from blood (without the need for a culture step) due to a number of scientific/medical, technical and commercial reasons. The main scientific/medical concerns arise from the fact that bacterial DNA can be present in blood, but may be unrelated to the active infection, if any (Struelens 2010). Bacteria can be introduced into the blood stream by activities ranging from brushing teeth to draining abscesses. The immune system usually curtails such minor, transient infections, but DNA from these bacteria can remain and give rise to false positives for molecular tests. Current molecular tests have also been reported (Mancini et al. 2010) to have a number of technical limitations such as (a) fairly high detection limits (often $>10^{3} \mathrm{CFU} / \mathrm{ml}$ ); (b) false negatives due to PCR inhibitors in the blood sample, or due to the presence of organisms not on the "included" list; (c) false positives due to contaminant bacterial DNA in reagents; and (d) the risk of carryover contamination among samples in the same extraction round. But perhaps the main reasons preventing the wider adoption of these molecular technologies are commercial. PCR based tests such as the Septi-Fast ${ }^{\mathrm{TM}}$ from Roche cost about $\$ 250$ per test (Mancini et al. 2010), and prices are unlikely to decrease substantially due to the high cost of ultra-pure chemicals needed to conduct the tests. On the other hand, blood cultures, which cost only $\$ 20-30$ per test, serve as a reliable and commercially effective way to screen a large number of patients that may have septicemia/sepsis. The most critical drawback of blood cultures is the long time needed to obtain positive results (the long TTP): one that the work presented here (a novel 
electrical-based system to detect the presence of viable bacteria in suspensions) seeks to ameliorate.

Since an increase in bacteria due to proliferation would result in an increase in the bulk capacitance value, we monitor the variation of the Bulk Capacitance with time, and if a significant increase is recorded, it is taken to imply the presence of viable bacteria in a suspension. We also previously showed the ability of this electrical method to detect bacteria in nutrient media (Tryptic Soy Broth) and food samples (apple juice and milk) with initial loads ranging from $\sim 1$ to $\sim 1000 \mathrm{CFU} / \mathrm{ml}$, in 6 to 0.5 hours, respectively (Puttaswamy and Sengupta 2010a). This represented a 4-10 fold improvement over methods/instruments analogous to Automated Blood Culture systems like the BACTE ${ }^{\mathrm{TM}}$, BacT/alert ${ }^{\mathrm{TM}}$ etc. In the present work, we seek to extend this method to blood cultures.

\subsection{Materials and Methods}

\section{$\underline{6.3 .1}$ Overview}

The experimental protocol that we use to validate our method, and compare it to standard blood cultures, is shown schematically in Figure 6.1. As shown, overnight logcultures of E. coli are centrifuged and the pellet obtained re-suspended in PBS to obtain a suspension with a concentration of $\sim 10^{9} \mathrm{CFU}$ of $E$. coli $/ \mathrm{ml}$. This is serially diluted to obtain concentrations of $\sim 10^{4}, \sim 10^{3}, \sim 10^{2}, \sim 10 \mathrm{CFU} / \mathrm{ml}$ in PBS. Exactly $0.1 \mathrm{ml}$ of these suspensions are then added to $0.9 \mathrm{ml}$, each, of sterile human blood to obtain "infected blood" with concentrations of $\sim 10^{3}, \sim 10^{2}, \sim 10, \sim 1 \mathrm{CFU} / \mathrm{ml}$. Two $1 \mathrm{ml}$ samples of "infected blood" (each with the same known bacterial load) are taken, one set introduced 
into tubes containing $10 \mathrm{ml}$ of BACTEC Ped-Plus ${ }^{\mathrm{TM}}$ media each and the other set introduced into BACTEC Ped-Plus ${ }^{\mathrm{TM}}$ bottles. The latter is loaded into a BACTEC 9240 Automated Blood-Culture System and the Times to Positivity (TTP) recorded. The former is assayed electrically using our method (described below). Appropriate controls, consisting of BACTEC bottles inoculated with $1 \mathrm{ml}$ of sterile blood, are also included with each run. 


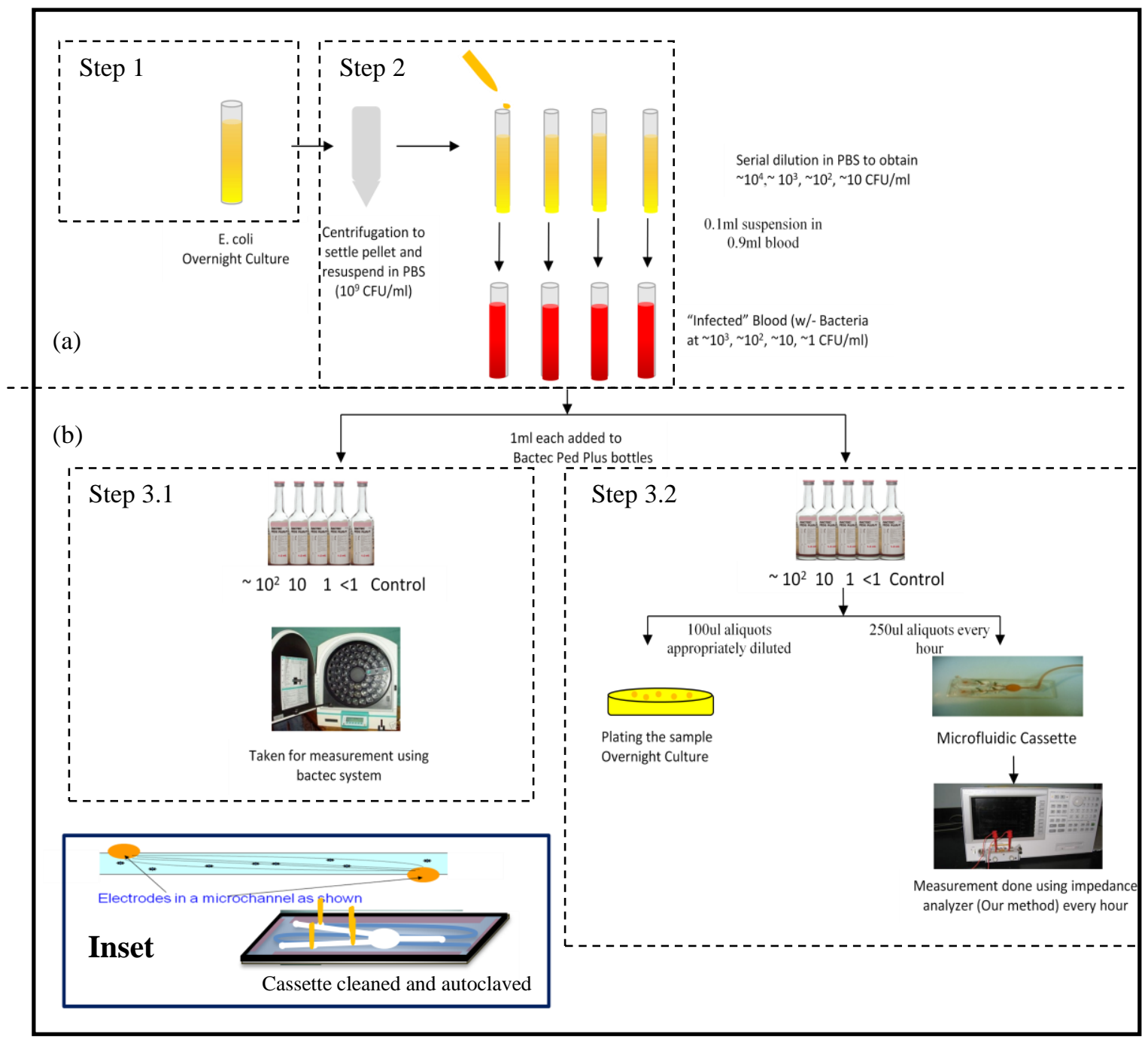

Figure 6.1: Blood Culture experiment overview

Overview of the experimental protocol: As seen, the protocol consists of (a) preparation of synthetic "infected blood" with known loads of bacteria (Step 1 to 2) (b) assaying parallel samples using BACTEC (Step 3.1) and our proposed method (Step 3.2).

Briefly, our method involves incubating the tubes with shaking in a standard incubator-shaker, periodically (once every hour) drawing a $250 \mu 1$ aliquot, introducing the aliquot into a microfluidic cassette with electrodes at specified locations, measuring Electrical Impedance $(\mathrm{Z})$ at multiple frequencies $(\omega)$ from $1 \mathrm{kHz}$ to $100 \mathrm{MHz}$, and using the $\mathrm{Z}$ vs. $\omega$ data to obtain a parameter that reflects the capability of the interior of the 
solution to store charge when subjected to an alternating electric field. An increase in the number of bacteria due to proliferation is expected to result in an increase in this parameter. Conversely, a "significant" increase in the value of this parameter is taken to imply the presence of viable bacteria in the sample, and when such an increase is observed, the sample is deemed positive by our method.

To verify that the increase in our parameter of interest corresponds to true increases in the number of viable bacteria, we also drew parallel aliquots from the sample at the time the electrical measurements were taken, plate them out (after serial dilution, if needed) and record the colony counts. Details of the various individual steps are provided below.

\subsubsection{Preparation of the Microfluidic Cassette}

A microfluidic cassette with a defined geometry is critical for obtaining impedance measurements with desired characteristics. The microfluidic cassette (shown in Figure 6.1) consists of a reservoir and two arms, each $4 \mathrm{~cm}$ long, $800 \mu \mathrm{m}$ deep and $1 \mathrm{~mm}$ wide. Gold coated crimp-pin electrodes are located $1 \mathrm{~cm}$ apart and on the opposite side of the channels, as shown in the figure. These cassettes are fabricated using Liquid Phase Photo-Polymerization of a commercially available UV curable polymer (Loctite $363^{\mathrm{TM}}$ ), a process that has been described in detail elsewhere (Sengupta et al. 2006a; Sengupta et al. 2004a). Prior to use, the cassettes are sterilized by autoclaving at $121^{\circ} \mathrm{C}$ and 15 psi. After each reading is taken during the course of an experiment, they are thoroughly flushed and rinsed with sterile PBS and dried in a sterile environment. After each experiment (consisting of a set of impedance measurements of aliquots drawn from 
the same source once every hour), the cassettes are washed thoroughly with soap, bleach, alcohol and water, and finally autoclaved for reuse.

\section{$\underline{6.3 .3}$ Sample Preparation (Step 1)}

Escherichia Coli $(\mathrm{DH} 5 \alpha)$ cultures used in this study were originally obtained from Invitrogen (Carlsbad, CA). Tryptic Soy Broth (TSB) powder was obtained from Bacto $^{\mathrm{TM}}$, Becton Dickinson (Sparks, MD). A 30g/l solution of the same is prepared and sterilized by autoclaving at $121^{\circ} \mathrm{C}, 15 \mathrm{psi}$. After cooling to room temperature, this sterile TSB solution is inoculated with the E Coli and incubated overnight at $37^{\circ} \mathrm{C}$. At the end of the incubation period (after log growth), a suspension with bacteria at $\sim 10^{9} \mathrm{CFU} / \mathrm{ml}$ is obtained.

\subsubsection{Sample Inoculation (Step 2)}

One milliliter of the bacterial suspension containing $\sim 10^{9} \mathrm{CFU} / \mathrm{ml}$ bacteria is taken in an Eppendorf tube and centrifuged for 8 minutes at $6000 \mathrm{rpm}$. The supernatant is discarded, and the pellet (bacteria) is re-suspended in an equal volume of PBS. The resulting suspension is serially diluted to obtain suspensions with concentrations of $\sim 10000, \sim 1000, \sim 100$, and $\sim 10 \mathrm{CFU} / \mathrm{ml}$. Then $0.1 \mathrm{ml}$ of these suspensions are then added to $0.9 \mathrm{ml}$ volumes of freshly drawn sterile human blood to obtain "infected blood" with concentrations of $\sim 1000, \sim 100, \sim 10$ and $\sim 1 \mathrm{CFU} / \mathrm{ml}$, respectively. 


\subsubsection{Blood Culture Protocol (Steps 3.1 and 3.2)}

One set of $1 \mathrm{ml}$ sample of "infected blood" (with known initial loads of bacteria), each, is added into separate tubes containing $10 \mathrm{ml}$ of BACTEC Ped-Plus ${ }^{\mathrm{TM}}$ media. Into an additional tube, $1 \mathrm{ml}$ of sterile blood is added to serve as control. Another set is obtained by adding, each, $1 \mathrm{ml}$ sample of blood ("infected samples" and a control) to separate BACTEC PEd-Plus ${ }^{\mathrm{TM}}$ bottles. The latter is loaded onto a BACTEC $^{\mathrm{TM}} 9240$ Blood Culture System, where they are monitored using the established method (briefly, this method consists of monitoring the sample, consisting of blood and microbial growth media, for an increase in the amount of dissolved $\mathrm{CO}_{2}$. When a detectable change in the $\mathrm{CO}_{2}$ level is detected within a bottle, it is flagged as positive, and the users notified by an alarm). Times to Positivity (TTP) for all samples are recorded, and samples are deemed negative after 5 days if they did not turn positive (as judged by the BACTEC measurement system/software) by then. The other set is incubated on a shaking platform (Fisher Scientific Nutating Mixer) within an incubator (Fisher 637D Isotemp Incubator) for 8 hours at $37^{\circ} \mathrm{C}$. Every 1 hour, $250 \mu$ of the sample is taken and injected into the microfluidic cassette for impedance measurements (these impedance measurements are then analyzed offline, as described later). After completing each measurement, the cassette is thoroughly flushed / rinsed with sterile PBS, and dried within a Biological Safety Cabinet. Simultaneously, every hour, $100 \mu 1$ aliquots of the sample is taken, diluted appropriately and plated onto petri-dishes to yield an estimate of the actual concentration of bacteria in the sample at that point in time. A total of 12 (non-control) samples were run, 3 samples each of blood with bacterial loads of $\sim 1, \sim 10, \sim 100, \sim 1000 \mathrm{CFU}$ in $1 \mathrm{ml}$ of blood. 


\subsubsection{Multi-frequency Electrical Impedance Measurements}

When the cassettes are loaded with an aliquot from the sample being investigated, the suspension fills the micro-channels in which electrodes are positioned as shown in Figure 6.1. The gold-coated electrodes on the cassette are connected to an Agilent 4294A Impedance Analyzer (Agilent technologies, CA, USA) via a 16047E connector that allows measurement of Impedance (Z), at multiple frequencies $(\omega)$ between $40 \mathrm{~Hz}$ and $110 \mathrm{MHz}$. We acquire the values $\mathrm{Z}$ at 500 frequencies between $1 \mathrm{kHz}$ to $100 \mathrm{MHz}$.

The Impedance $(\mathrm{Z})$ is the vector ratio of the Applied AC Voltage $(\mathrm{V})$ and the Measured AC Current (I) $(Z=V / I)$. By definition,

$$
\mathrm{Z}=\mathrm{R}+j \mathrm{X}
$$

where

$$
\begin{aligned}
& \mathrm{R}, \text { the Resistance, is the "In-phase" component, } \\
& \mathrm{X} \text {, the Reactance, is the "Out of Phase" component, and } \\
& j=\sqrt{-1}
\end{aligned}
$$

To obtain the Impedance at a given frequency, Impedance Analyzer generates a $500 \mathrm{mV}$ (peak to peak) voltage at that frequency, and records the magnitude and phase of the AC current through the sample as result of the application of the same. It also takes as inputs the frequency range to be scanned ( $1 \mathrm{kHz}$ to $100 \mathrm{MHz}$ in our case) and the number of points desired (500, the maximum possible, in our case) and selects logarithmically equi-spaced frequencies. It records the measured values of $\mathrm{R}$ and $\mathrm{X}$ corresponding to each frequency $(\omega)$ in an ASCII file. The data in this file are analyzed off-line. 


\section{$\underline{\text { 6.3.7 }}$ Circuit Model and Data Analysis}

The microfluidic system consisting of an aqueous solution in a microfluidic channel with electrodes on either end can be represented by an equivalent electrical circuit as shown in Figure 6.2(a) (Puttaswamy and Sengupta 2010a; Sengupta et al. 2005a). Different components in the circuit arise due to the different effects of the applied electric field. The flow of ions through the solution (and the viscous resistance to the same) is accounted for by the bulk resistance $\left(R_{b}\right)$; the interfacial resistance $\left(R_{e}\right)$ and inductance $\left(\mathrm{L}_{\mathrm{e}}\right)$ are those of the electrodes themselves and that of the wires connecting them to the impedance analyzer; the electrochemical "double layers" formed on the surfaces of charged metal electrodes are accounted for by the Interfacial Capacitance $\left(\mathrm{C}_{\mathrm{e}}\right)$; and the charges stored in the interior of the suspension is accounted for the Constant Phase Element (CPE). In comparison to our previous circuit model as in Chapter 5, we have an additional circuit element $\mathrm{L}_{\mathrm{e}}$, which accounts for the inductance due to the wired used for the connection. The use of $\mathrm{L}_{\mathrm{e}}$ has shown to decrease the sum of squares error during the fit of the circuit model to the data. A CPE, rather than an ideal capacitor is used because the charge-storing behavior of bacteria and other cells is expected to be different from that of an ideal capacitor. 


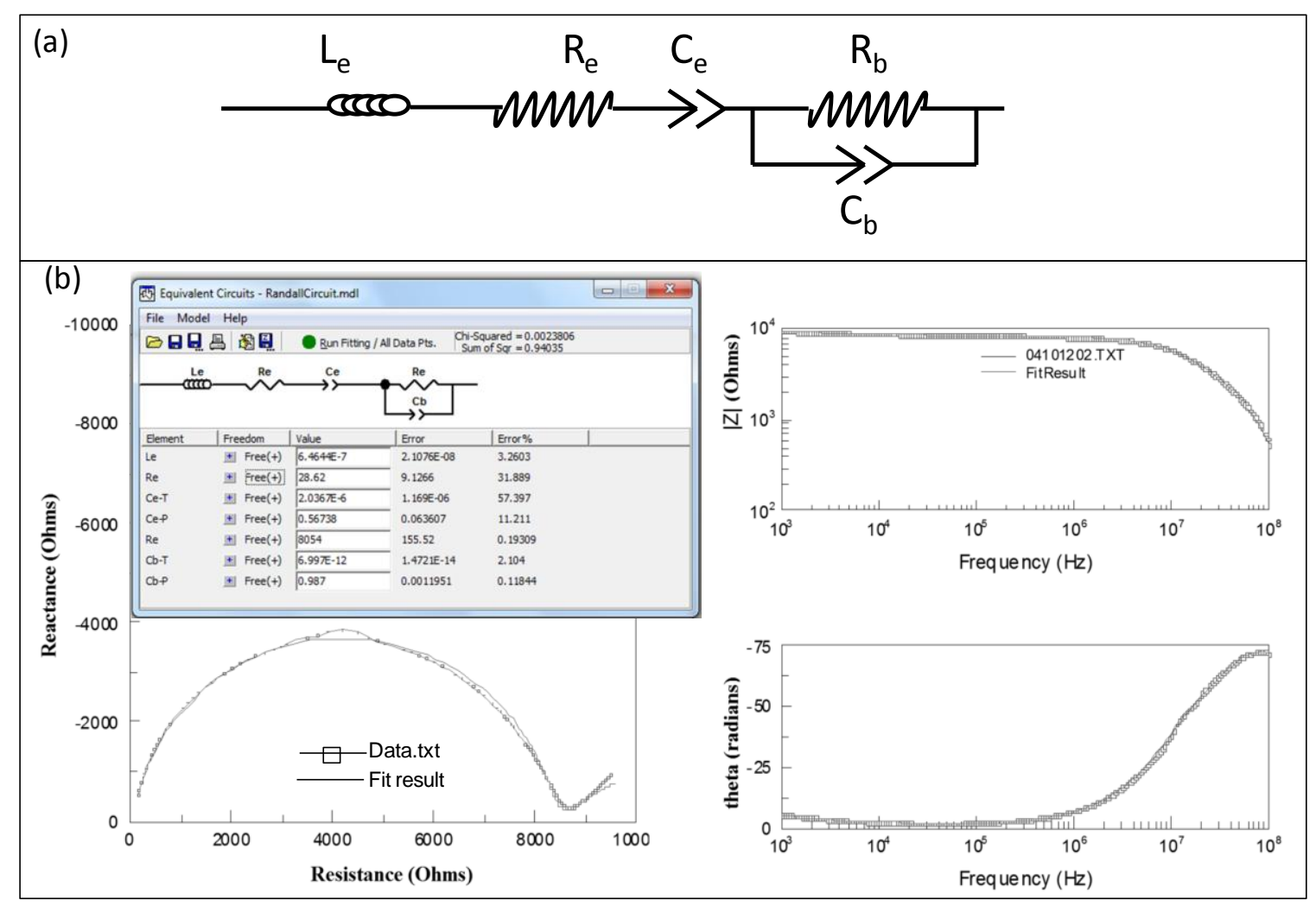

Figure 6.2: ZView ${ }^{T M}$ analysis with circuit used for blood cultures

(a) Equivalent circuit diagram as used in the $Z$ view $^{T M}$ software for analysis (b) Results obtained using ZView ${ }^{T M}$. The data from the impedance analyzer (represented by red line with dots) is fitted to the circuit model shown on the right (represented by green line without dots) with individual values of all parameters given below the circuit diagram.

The CPE is a non-intuitive circuit element that replaces a capacitor in a circuit when the there is some type of non-homogeneity in the system, delaying or impeding the movement of charge carriers ( Barsoukov and MacDonald 2005). Mathematically, a CPE is an element whose impedance is given by the equation

$$
Z=0-j\left(\frac{1}{(w Q)^{n}}\right)
$$


One may also note that if the Phase-Angle of the CPE (CPE-P) ( $\mathrm{n}$ in the above equation) equals 1 then the impedance of the element is identical to that of an ideal capacitor with the same magnitude.

Data obtained from the Impedance Analyzer is fit to the equivalent circuit shown in Figure 2(a) (displayed using the software $\mathrm{ZView}^{\mathrm{TM}}$ ). The software is written for use in Electrical/ Electrochemical Impedance Spectroscopy. It accepts as input measured values of Resistance (R) and Reactance (X) at multiple frequencies (or, equivalently, the magnitude of the Impedance $(\mathrm{Z})$ and the phase angle $(\theta)$ ), allows the user to propose an equivalent circuit for the material being investigated, and provides an estimate of the values of the individual elements in the equivalent circuit $\left(\mathrm{L}_{\mathrm{e}}, \mathrm{R}_{\mathrm{e}}, \mathrm{C}_{\mathrm{e}}, \mathrm{R}_{\mathrm{b}}, \mathrm{CPE}-\mathrm{T}\right.$, and CPE-P in our case), along with an "error" of the estimate. By providing estimates of each individual parameter, the software allows the user to distinguish changes to the overall impedance occurring due to an increase in the bulk capacitance, from other factors, such as changes to the bulk resistance (temperature swings result in changes to the electrical conductivity of the solution, which in turn changes the bulk resistance).

\section{$\underline{\text { 6.3.8 }}$ Tracking CPE-T Values, and Obtaining Times to Positivity (TTPs)}

The ZView ${ }^{\mathrm{TM}}$ software generates not only an estimate of the parameters (such as the Bulk Capacitance CPE-T value), but also an "error". For two recorded values to be "significantly" different from each other, there should not be any overlap between the ranges ([value - error], [value + error]) of the two readings. As we calculate the value of the Bulk Capacitance CPE-T after various intervals of time, we examined whether or not the present value is "significantly" greater than the initial (0-hour) value. A "significant" 
increase in the CPE-T value would indicate an increase in the number of species capable of storing charge that are dispersed in the medium. Since other such species (such as proteins and blood cells) are not expected to increase in number, such an increase can only be brought about by bacterial proliferation, as is hence taken as a signature of the presence of viable bacteria in the sample. The time taken to obtain such a "significant" increase in the value of the CPE-T is our Time to Positivity.

The use of this criterion is illustrated in Figure 6.3. As seen, initially (at $t=0$ ), the value of the CPE-T parameter (represented by blue diamonds) is $3.8 \pm 0.04 \mathrm{pFs}^{\mathrm{n}-1}$. The range of values is represented by the error bars. The value of CPE-T after 1 hour of incubation (obtained based on Impedance measurements taken at that time) is $3.85 \pm$ $0.04 \mathrm{pFs}^{\mathrm{n}-1}$. As seen, the range overlaps with that of the 0 -hour value (red shaded region). Thus, while we know (from the colony counts of aliquots drawn at the time the impedance measurements are taken) that the number of bacteria increased (from $~ 360$ to $\sim 460 \mathrm{CFU} / \mathrm{ml}$ of suspension), the increase did not cause a "significant" change in the value of the CPE-T. However, the CPE-T value at the 2 hour mark $3.89 \pm 0.04 \mathrm{pFs}^{\mathrm{n}-1}$ is "significantly" different from the 0-hour value (error bars fall outside the red-shaded region). Thus, 2 hours is our Time to Positivity (TTP) for this sample. During the course of the experiment, the value of CPE-T changed by $13.5 \%$, while the other parameters such as bulk resistance $\left(\mathrm{R}_{\mathrm{b}}\right)$ changed by less than $3 \%$ and the electrode parameters did not change significantly. Moreover, at the time to positivity, the change in $\mathrm{R}_{\mathrm{b}}$ was $<0.8 \%$ while CPE-T increased by $2.5 \%$. 


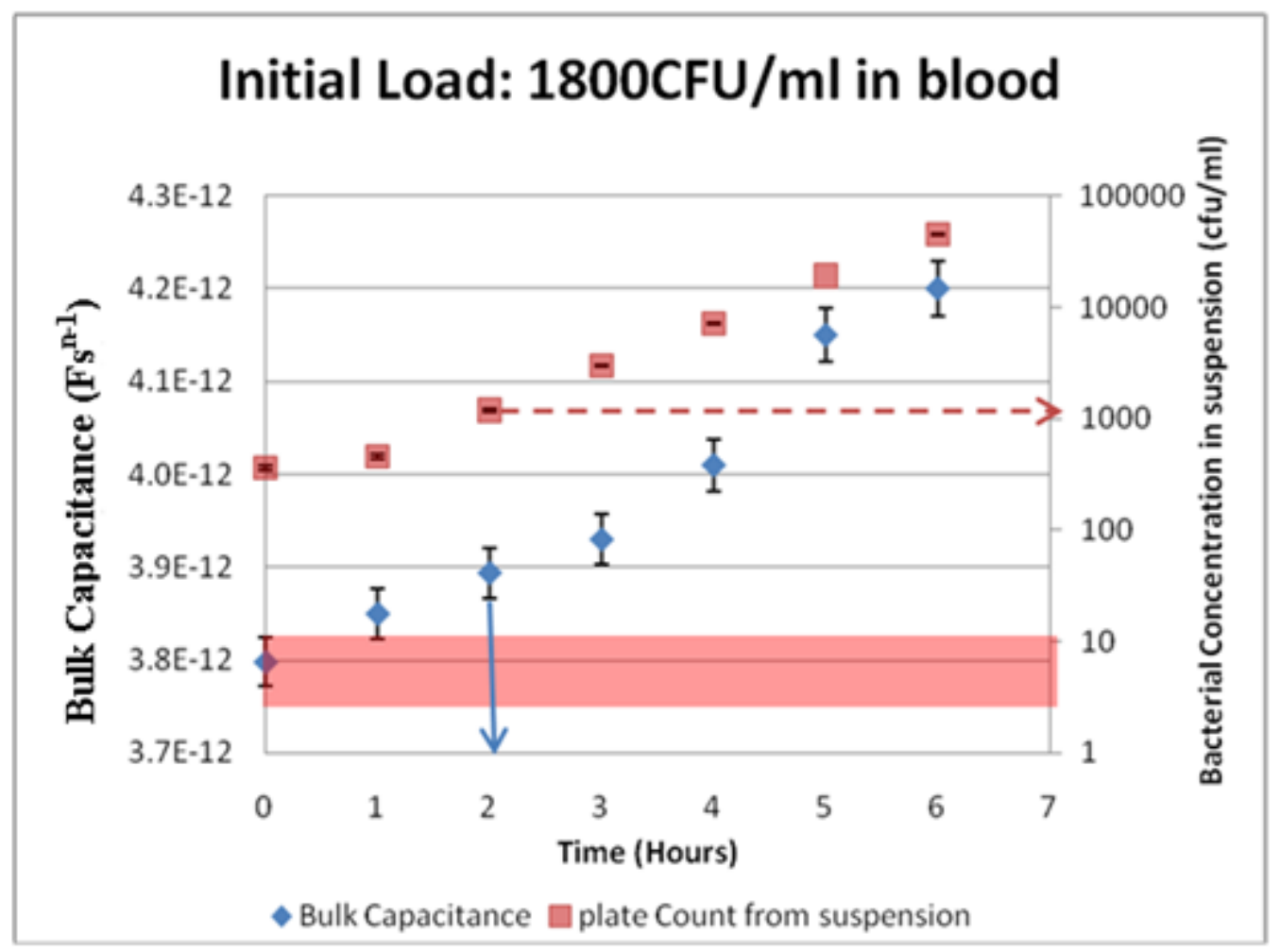

Figure 6.3: Bulk Capacitance vs. time plot for E.coli in blood

Plot of the increase in the bulk capacitance (Blue diamonds) with actual increase in the concentration of the bacteria (red squares) in the suspension vs. time. The plot also indicates the time to detection (shown by the solid blue arrow) and the concentration of bacteria in suspension (containing blood with media) at the time of detection (shown by dotted red arrow). For this sample, the error bar of CPE-T value of 2 hours does not overlap with the error bar of zero-hour reading and hence 2 hours is considered as the Time to Positivity

\subsection{Results}

For all "Blood Cultures", each containing $1 \mathrm{ml}$ of "infected" blood with a known bacterial load (or uninfected control) dispersed into 10ml of BACTEC PED-PLUS ${ }^{\mathrm{TM}}$ media, aliquots are drawn and CPE-T values (reflecting the ability of the bulk suspension to store charge) at various points in time are calculated as explained earlier. These values are then plotted and, as explained earlier, a "significant" increase in the value of the CPE- 
$\mathrm{T}$ parameter is taken as a signature of the presence of viable and proliferating bacteria in the original sample. In Figure 6.4, we present some additional sample data showing the rise of the value of CPE-T in concert with the numbers of bacteria in the sample (as revealed by plate counts) for different samples with different initial loads of viable bacteria. 

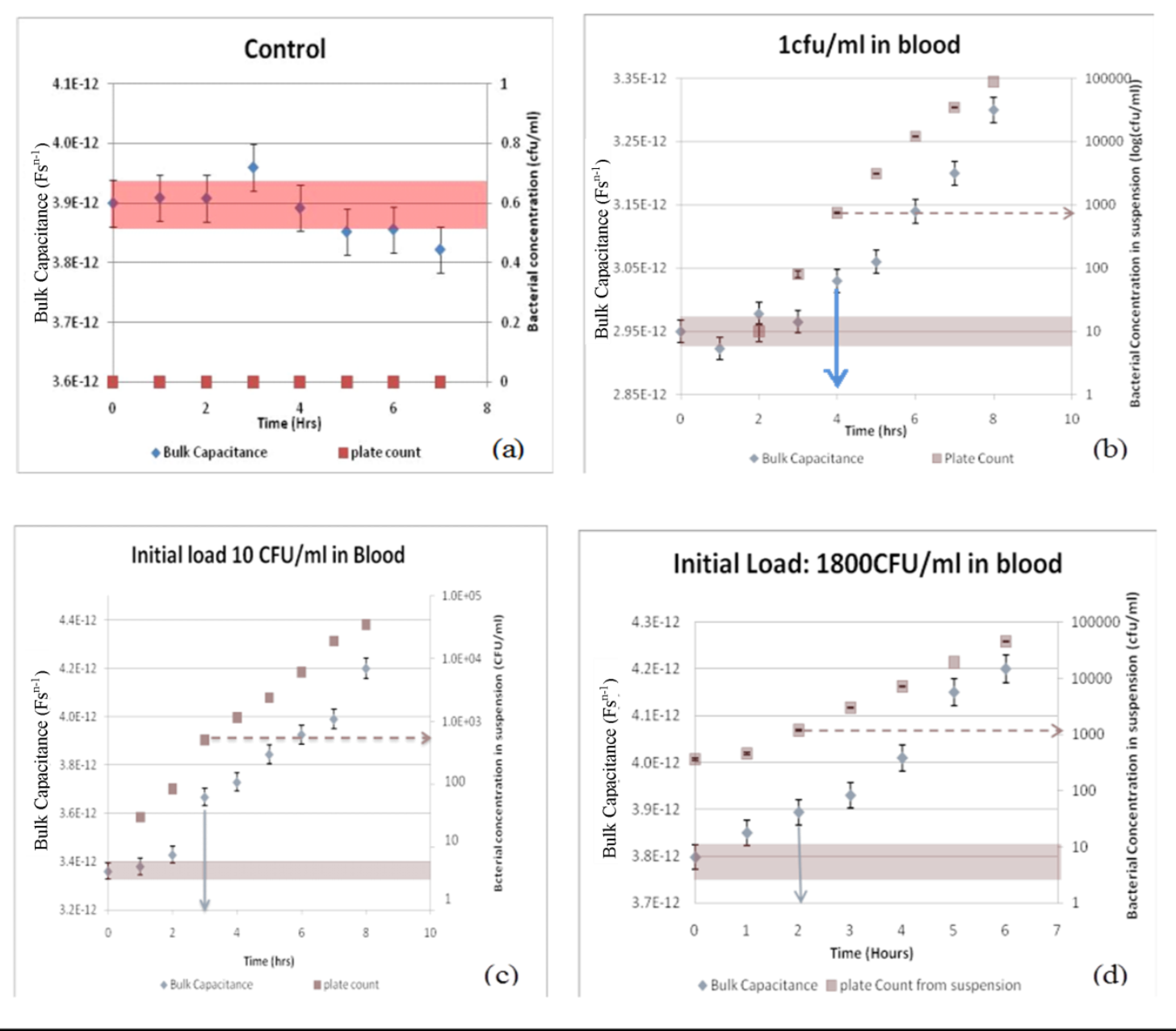

Figure 6.4: Plots of Bulk capacitance vs. time for E.coli in blood at different

concentrations

Typical plots showing how the calculated value of the CPE-T (the "bulk capacitance" parameter) changes with time for different samples (with different initial loads). Also shown are plate counts obtained from aliquots drawn at the same time at which the impedance measurements are taken. Samples turn "positive" when the recorded value of CPE-T is significantly different from the initial (0-hr) value. It is also observed that bacterial concentrations at Time to Positivity are $1000-10,000 \mathrm{CFU} / \mathrm{ml}$.

As in Figure 6.3, the blue solid arrows indicate the Time to Positivity (TTP) for all plots. This is the hour at which the value of the CPE-T obtained first becomes significantly different from the zero hour value. The red dotted arrow, on the other hand, 
indicates the concentration of bacteria in the sample at TTP. As seen, this concentration, which we call the "Threshold Concentration" for the detection of bacteria in the sample, is observed to be $\sim 1000-10,000 \mathrm{CFU} / \mathrm{ml}$ for all measurements taken.

Because different samples start with different initial loads of bacteria, it takes different amounts of time for the bacterial loads in them to reach the "Threshold Concentration". In general, the lower the initial load, the longer it will take for the sample to turn positive. This is borne out for our experiments, as summarized in Table 1 and displayed graphically in Figure 6.5. As can be seen, this inverse relationship between TTP and initial load is also valid for the BACTEC and has been documented previously by other researchers as well (Haimi-Cohen et al. 2002). This is to be expected since the BACTEC relies on detecting $\mathrm{CO}_{2}$ produced by the bacteria, and this can be detected only when the number of $\mathrm{CO}_{2}$ producing bacteria in the sample reaches a certain threshold. 

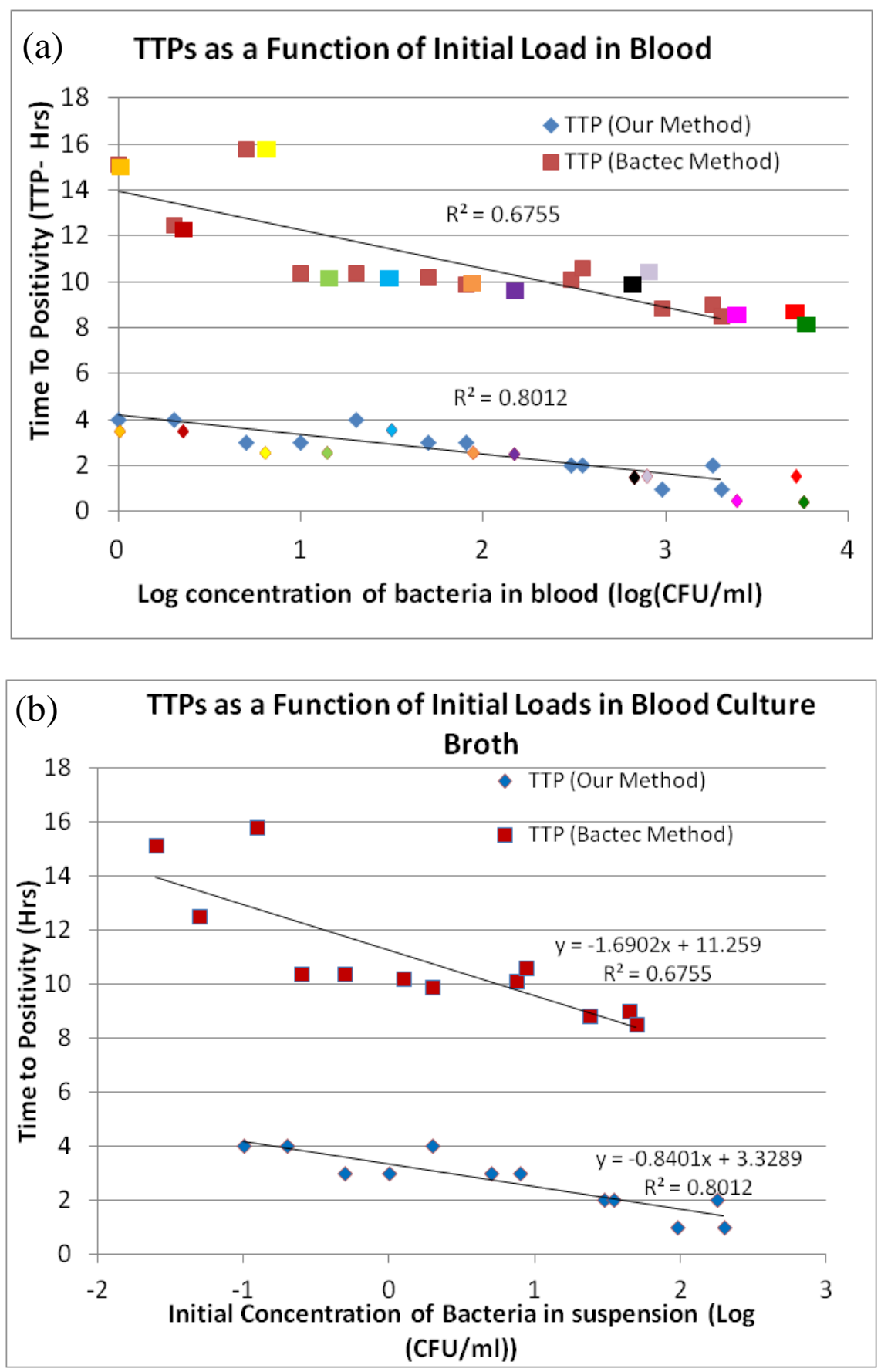

Figure 6.5: Plot of TTD as a function of Initial load of bacteria

(a) Shows the consolidated plot of TTPs obtained by our method of detection and that of BACTEC method for the same given initial concentrations of bacteria in blood. Pairs, consisting of one sample introduced into BACTEC and another containing the same initial load assayed using our method, are represented by the same color (b) Shows the consolidated plot of TTPs obtained by our method and BACTEC for the initial concentrations of bacteria in blood culture broth (containing infected blood in BACTEC Ped-Plus ${ }^{T M}$ Media). 


\begin{tabular}{ccc}
\hline $\begin{array}{c}\text { Initial Load in } \\
\text { Blood }\end{array}$ & TTP (Our Method $)$ & TTP $\left(\right.$ BACTEC $\left.^{\mathrm{TM}}\right)$ \\
$(\mathbf{C F U} / \mathrm{ml})$ & & \\
\hline $\mathbf{1 - 5}$ & 4 & $12.5-15.8$ \\
& $3-4$ & $10.2-10.4$ \\
$\mathbf{1 0 - 5 0}$ & $2-3$ & $9-10$ \\
$\mathbf{8 0 - 3 5 0}$ & $1-2$ & $8.5-9.01$ \\
\hline $\mathbf{9 5 0 - 2 0 0 0}$ & & \\
\hline
\end{tabular}

Table 6.1: TTP comparison of our method vs. BACTEC

The table shows the actual values of TTPs obtained by using our method and BACTEC with respect to initial concentration of bacteria in blood.

For both our system and for the BACTEC, the Time to Positivity (TTP) is related to the initial and threshold concentrations by the equation

$$
\begin{gathered}
\text { TTP }=1.443 \mathrm{t}_{\mathrm{D}} \ln \left(\mathrm{n}_{\mathrm{T}} / \mathrm{n}_{0}\right) \ldots \ldots \ldots \ldots \ldots \ldots \ldots \ldots \ldots \ldots \\
\text { where } \mathrm{n}_{\mathrm{T}} \text { is the Threshold Concentration, } \\
\mathrm{n}_{0} \text { is the Initial Concentration, and } \\
\mathrm{t}_{\mathrm{D}} \text { is the Doubling time of the bacteria. }
\end{gathered}
$$

This equation may be re-written as

$\mathrm{TTP}=3.32 \mathrm{t}_{\mathrm{D}} \log _{10}\left(\mathrm{n}_{\mathrm{T}}\right)-3.32 \mathrm{t}_{\mathrm{D}} \log _{10}\left(\mathrm{n}_{0}\right)$ 
Thus, it is possible to estimate both the doubling time of bacteria in our system and the threshold values from the equations of the lines of fit to the data displayed in Figure 6.5(b).

For our method, we obtained a doubling time of $\sim 15 \min$ (90\% confidence interval of [11 min, $20 \mathrm{~min}])$ and Threshold Concentration of $10^{3.96} \mathrm{CFU} / \mathrm{ml}$ (90\% confidence interval of $\left.\left[10^{3.37} \mathrm{CFU} / \mathrm{ml}, 10^{5.01} \mathrm{CFU} / \mathrm{ml}\right]\right)$ for bacteria in the blood. The corresponding values for the BACTEC are $\sim 31 \mathrm{~min}$ for the doubling time (90\% CI of [18 $\mathrm{min}, 43 \mathrm{~min}]$ ) and $10^{6.66} \mathrm{CFU} / \mathrm{ml}$ for the Threshold Concentration $\left(90 \% \mathrm{CI}\right.$ of $\left[10^{5.08} \mathrm{CFU} / \mathrm{ml}, 10^{10.30}\right.$ $\mathrm{CFU} / \mathrm{ml}])$.

Thus, our calculated Threshold Concentration for BACTEC is similar to earlier estimates (of it being $\sim 10^{8} \mathrm{CFU} / \mathrm{ml}$ (Smith et al. 2008) ) and the doubling time of the bacterium estimated from the line of fit to the BACTEC data $(\sim 30 \mathrm{~min})$ are also in line with earlier reports of $E$. coli growth rates (doubling times of 20-30 min) (Cooper and Helmstetter 1968). However, the values for the doubling time obtained from the TTD data of our method appear on the low side (although the reported range in literature just barely overlaps with our $90 \%$ confidence interval). We speculate that this apparently low doubling time (for the same bacterium/growth-medium as for the BACTEC ${ }^{\mathrm{TM}}$ ) is the result of some of the bacteria reproducing within a short time of our cultures being initiated. Because of the low Threshold Concentration of our method (of $\sim 10,000$ $\mathrm{CFU} / \mathrm{ml}$ ), the bacteria go through with a relatively small number (5-15) of doubling cycles before the samples are flagged as positive. As a consequence, errors introduced into the calculated "average doubling time" as a result of the early reproduction of some 
bacteria, do not get sufficiently "smoothed over" by repeated doublings. The Threshold Concentration for our technique is dramatically lower (>1,000 times) in comparison to the BACTEC results and our TTPs are also being significantly (4-10 times) shorter.

\subsection{Discussion}

The results presented here show the ability of our impedance based technique to detect the presence of viable bacteria in blood samples significantly faster than BACTEC. In patients exhibiting Systemic Inflammatory Response Syndrome (SIRS, the prelude to Sepsis), infectious bacteria are typically present at 1-100 CFU/ml (Yagupsky and Nolte 1990). As shown in Table 6.1, we were able to detect $\sim 1 \mathrm{CFU} / \mathrm{ml}$ of $E$. coli in 4 hrs (as opposed to $\sim 16 \mathrm{hrs}$ using BACTEC) and as early as $2 \mathrm{hrs}$ for initial loads of $\sim 100$ CFU/ml (as opposed to $\sim 10 \mathrm{hrs}$ for BACTEC).

Such significant decreases in TTP are made possible by the fact that we monitor a parameter (charge stored in the interior of a suspension) that is much more sensitive to bacterial proliferation than parameters like $\mathrm{CO}_{2} / \mathrm{O}_{2}$ levels, $\mathrm{pH}$ conductivity, etc. that are monitored by current instruments like BACTEC, BacT/Alert, ESP system, etc. These monitored parameters change due to the cumulative effect of bacterial metabolism. But the rate of bacterial metabolism is inherently limited. Based on our knowledge that the specific oxygen consumption rate for $E$. coli is $20 \mathrm{mmol}$ of oxygen per hour per gram (dry weight) of bacteria (Andersen and von Meyenburg 1980a) and a typical bacterium has a dry weight $\sim 10^{-12} \mathrm{~g}$ (Borsheim et al. 1990a), we estimate that one E. coli bacterium

consumes only $2 \times 10^{-14}$ moles of oxygen in one hour. When one starts with low 
concentrations of bacteria ( $100 \mathrm{CFU} / \mathrm{ml}$ or lower), the amount of metabolite processed is extremely small ( $\sim 2 \times 10^{-12}$ moles of $\mathrm{O}_{2}$ in one hour). For a well-oxygenated medium (with a dissolved oxygen concentration of concentration $\sim 2 \times 10^{-4} \mathrm{M}$ ), this represents only a $0.00001 \%$ change in the $\mathrm{O}_{2}$ level in the medium as a whole. Such a change is too small to be discerned by virtually any sensor, and hence one has to wait for the cells to proliferate to $\sim 10^{8} \mathrm{CFU} / \mathrm{ml}$ (Smith et al. 2008) before a discernable change in the physical properties of the medium can be observed. This also implies that attempts to improve performance by building better sensors for metabolic products such as $\mathrm{CO}_{2}$ are only likely to produce marginal improvements at best. For instance, Garcia Rubio and coworkers have developed a technique based on multi-wavelength reflectance spectra that detects changes to the relative amounts of $\mathrm{O}_{2} / \mathrm{CO}_{2}$ based on optical properties of hemoglobin present in the sample (decrease of $\mathrm{O}_{2}$ leads to the conversion of oxyhemoglobin to hemoglobin). By essentially having the "sensors" (hemoglobin) dispersed in the media, this method reduces the TTP of cultures by $20 \%$, compared to BACTEC (Smith et al. 2008).

In contrast, our method relies on the fact that bacterial cells, when exposed to AC fields, store charge (Asami 2002), and that the amount of charge stored is more than 100 times greater than that stored by a equivalent amount of aqueous solution (Poortinga et al. 1999a). Thus, the value of the bulk capacitance is more sensitive to a change in the number of viable bacteria suspended. It is also self-normalizing. Different substrates, and even different samples of the same substrate, may start out with different values of the bulk capacitance due to a variety of reasons (e.g. different amounts of WBCs in blood drawn from different people). We can, however, use the exact same criterion (significant 
increase of $\mathrm{C}_{\mathrm{b}}$ from their respective 0 -hr values) to determine the presence of viable bacteria in all cases.

However, currently our system suffers from one major drawback, viz. it involves a lot of manual labor. This includes drawing aliquots from the sample being incubated every hour and introducing it into a microfluidic channel, taking impedance measurements at multiple frequencies, and analyzing the results offline using the ZView $^{\text {TM }}$ software. This not only prevents us from analyzing multiple samples simultaneously, but also from taking samples at more frequent intervals (we currently take these measurements only once an hour). The consequence of the low sampling rate is a longer TTP in many cases. For instance, as seen in Figure 6.4(d), at $\mathrm{t}=1 \mathrm{hr}$, the value of CPE-T is $3.85 \pm 0.039 \mathrm{pFs}^{\mathrm{n}-1}$, whose error bar overlaps marginally with that of $0 \mathrm{hr}$ reading $\left(3.81 \pm 0.038 \mathrm{pFs}^{\mathrm{n}-1}\right)$. If we had taken the next measurement a short while later (say 15 minutes), there is a high possibility that we could have detected a "significant change" in CPE-T at that time, in which case our TTP would have been $1.25 \mathrm{hrs}(1 \mathrm{hr}: 15$ min). However, due to limitations imposed by the use of manual labor, we could run the samples only at every 1 hour interval, and hence our observed TTP is higher ( $2 \mathrm{hrs}$ in this case).

However, all of the tasks currently done manually can potentially be automated, thereby overcoming the limitations mentioned above. The back end instrumentation will draw aliquots periodically, measure the impedance at multiple frequencies, calculate bulk capacitance, and display the results using a graphical user interface. It will also sound an alarm if the current value of the bulk capacitance parameter (CPE-T) is significantly greater than the 0-hour reading to alert the user, as is currently done by BACTEC, etc. 
when samples turn positive. When automated, more samples can be analyzed in parallel, and readings can be taken every 5 or 10 minutes instead of every hour, reducing the TTPs further. Our next efforts will focus on building such an automated system.

Once built, the system will be used to test synthetic cultures containing other, slower growing bacteria and "real world" samples, and its performance will be compared to that of the BACTEC, BacT/Alert and other Automated Blood Culture Systems. Even for current Automated Blood Culture Systems like the BACTEC, the TTP for blood cultures is a function of the metabolic rate of the bacteria, with bacteria having slower metabolic rates (longer doubling times) taking longer to be detected. For instance, it has been previously observed (Haimi-Cohen et al. 2002) that with initial loads of $\sim 1 \mathrm{CFU} / \mathrm{ml}$ of Staphylococcus epidermidis , the TTPs using BACTEC are $~ 19-24 \mathrm{hrs}$ (vs. 12-16 hrs for similar loads of $E$. coli, as obtained by us). The ratio of their TTPs ( 1.6) is virtually identical to that of their doubling times ( 35 minutes for $S$ epidermidis (König et al. 2001), 20 min for E. coli (Cooper and Helmstetter 1968) ). We expect TTPs to be proportional to the doubling time of the infecting bacteria for our system as well (as also indicated by Equation 6.1).

An important question that will have to be answered is how long the cultures need to be run for our method before a sample may be deemed negative. Currently, with BACTEC and other similar machines, cultures are run for 5 days (120 hours) [Although the feedback we received from clinical practitioners is that if a culture does not turn positive in 72 hours ( 3 days), it is unlikely to do so even after 5 days]. While we expect to achieve similar, 4-10 fold, reduction in the cutoff time as we did for the TTP and bring it down to $24 \mathrm{hrs}$ (as opposed to $120 \mathrm{hrs),} \mathrm{to} \mathrm{answer} \mathrm{this} \mathrm{question} \mathrm{effectively} \mathrm{and}$ 
unequivocally, we will have to conduct more extensive studies, including the use of "real world" clinical samples. Again, such studies will be attempted only after our method is automated.

To conclude, we would like to highlight that in this work, we have demonstrated the feasibility of using a novel electrical based technique to detect the presence of viable bacteria in blood cultures 4-10 times faster than currently possible using Automated Blood Culture systems like the BACTEC, BacT/Alert, etc. While the technique needs to be refined further and tested much more extensively before it can actually be used in a clinical setting, it represents a potential breakthrough technique that can dramatically reduce TTPs of blood cultures. 


\title{
- CHAPTER 7
}

\author{
NOVEL ELECTRICAL METHOD FOR THE RAPID \\ DETERMINATION OF MINIMUM INHIBITORY \\ CONCENTRATION (MIC) AND ASSAY OF
}

BACTERICIDAL/BACTERIOSTATIC ACTIVITY

The material presented in this chapter has been accepted to be published as "Novel Electrical Method for the Rapid Determination of Minimum Inhibitory Concentration (MIC) and Assay of Bactericidal/Bacteriostatic Activity"

Sachidevi Puttaswamy, Byung-Doo Lee, Banoo Amighi, Sounak Chakraborty and Shramik Sengupta

in Journal of Biosensors and Bioelectronics

\subsection{Abstract}

We present a rapid (4-hr) electrical method for Antibiotic Susceptibility Testing that not only yields the MIC of candidate antibiotics, but also simultaneously determines the antibiotic's effect on the bacteria (bactericidal/bacteriostatic). Unlike conventional "impedance microbiology" methods that rely on measuring the effects of bacterial metabolism on the conductance / impedance of the suspension at a single chosen frequency, our method uses measurements at 500 frequencies between $1 \mathrm{kHz}$ and 100 $\mathrm{MHz}$ to estimate the amount of electric charge stored due to charge-polarization at intact 
cell-membranes of living bacteria (the suspension "bulk capacitance"). By doing so, we are able to track the number of live bacteria in suspensions as the observations are taken every 1 hour. It thus determines whether the numbers of viable bacteria present is increasing (bacteria proliferating in presence of antibiotic), decreasing (bacteria being killed) or holding steady (bacterial numbers held static). Three well-characterized bacterial strains (E. coli ATCC-25922, S. aureus ATCC-29213 and P aeruginosa ATCC27853 ) were tested against a range of concentrations ( 0 to $128 \mathrm{mg} / \mathrm{l}$ ) of known static and cidal antibiotics. For each sample (bacterial strain at a given concentration of antibiotic), statistical analysis of the "bulk capacitance" values, recorded over 4 hours was used to determine whether the bacteria were proliferating, being killed, or being held static. The minimum concentration of antibiotic for which the bacteria were killed or failed to proliferate is considered the Minimum Inhibitory Concentration (MIC). MICs obtained fell within the expected range for the strains tested, and "static" and "cidal" antibiotics were correctly identified. This method thus demonstrates the potential to provide in $4 \mathrm{hrs}$, clinically relevant information such as the MIC of bacterial strains (that currently take up to 2 days) and the mode of action (static/cidal) that currently takes an additional day.

\subsection{Introduction}

In a number of clinical situations, knowing the antibiotic susceptibility profile of the particular pathogen causing the infection (in particular the MIC of various candidate antibiotics) can help determine the optimum treatment protocol. For instance, it has been reported that for certain antibiotics ( $\beta$-lactams, macrolides, clindamycin and linezolid) the 
clinical efficacy is strongly correlated with the duration for which their concentrations in the serum was above their respective MICs, whereas for others (aminoglycosides and fluoroquinolones), the ratio of the peak serum concentration to the MIC is the major determinant of efficacy (Craig 2001). In certain, more critical situations (such as endocarditis and meningitis), it may be desired to administer antibiotics at bactericidal doses that kill the infecting organism, as opposed to bacteriostatic doses that merely prevent the organism from proliferating further (Finberg et al. 2004). In other cases, such as streptococcal and clostridial gangrene, it is more preferable to use bacteriostatic drugs, since cidal drugs can cause the dying cells to release internal toxins, which may further aggravate the morbidity (Finberg et al. 2004). Since drugs that are bactericidal for one organism may be bacteriostatic for other organisms, or other strains of the same organism (Finberg et al. 2004), it is not always possible to predict the mode of action for a given antibiotic.

Further, it can take a relatively long time (up to 2 days) to obtain MICs of candidate antibiotics for a particular strain (Chen et al. 2010), and an additional day to obtain information regarding the bactericidal/bacteriostatic nature of a given antibiotic's activity at various concentrations above its MIC (Sule et al. 2011). Cutting down the time needed to determine MICs and to assay for bacteriostatic/bactericidal activity could help the clinician formulate more effective treatment protocols and achieve better patient outcomes. In addition, a method that simultaneously indicates the mode of action of the antibiotic (cidal or static) could be of added value to the clinician.

MICs are currently determined using Disc-diffusion or Broth-dilution (macro or micro) methods. While Disc-diffusion remains a largely manual technique, broth-dilution 
(esp. micro-dilution) has been automated in the recent past to reduce labor costs and preparation time, and has emerged as the technique of choice for large clinical microbiology labs. These automated Antibiotic Susceptibility Testing (AST) instruments rely on a variety of methods to determine the occurrence of bacterial proliferation (or lack thereof) in the presence of various concentrations of the candidate antibiotics. For instance, VITEK $^{\mathrm{TM}}$ (from Biomeriux) uses an increase in solution turbidity as a measure of an increase in bacterial concentration (Jorgensen and Ferraro 1998), whereas the Phoenix $^{\mathrm{TM}}$ (Becton-Dickinson) and the Microscan WalkAway ${ }^{\mathrm{TM}}$ (Dade Microscan) systems use flourimetric/colorimetric methods to detect ongoing metabolism (redox reactions) (Horstkotte et al. 2004) (Jorgensen and Ferraro 1998). These systems suffer from two major drawbacks. Firstly, the time needed to obtain susceptibility profiles is still rather long, typically 12-24 hours (Jorgensen and Ferraro 1998; S. Whittier 2006). Secondly, they do not tell the user whether the action of the antibiotic is bacteriostatic or bactericidal.

Various other approaches are being investigated to further reduce the time needed to obtain MIC values. Newer methods being developed include those using Dielectrophoresis (DEP) (Chung et al. 2012), Microfluidic incubation (Chen et al. 2010), magnetic bead rotation sensors (Kinnunen et al. 2011). However each of these methods has its own limitations. The DEP-based method assays for the effect of the antibiotic by monitoring elongation of the bacterial cells (that caused a change in their DEP properties). This method was demonstrated for $\beta$-lactam antibiotics on gram-negative bacteria (E. coli, and Klebsiella pneumoniae). It is thus very specific, and possibly cannot be generalized to all antibiotic-bacteria combinations. The method based on sensing 
Magnetic-bead rotation needs an antibody specific to the bacterium being investigated to be conjugated to the magnetic beads, using which bacteria of interest adhere to the magnetic beads (sensing platforms), again making an individual test specific to a particular bacterium. It may be noted that both VITEK ${ }^{\mathrm{TM}}$ and Pheonix ${ }^{\mathrm{TM}}$ systems do not require prior knowledge of bacterial ID, allowing AST to be performed in parallel with bacterial identification. Time saved by using these methods would be of less clinical value if they can be initiated only after the infecting bacteria have been identified. The microfluidic incubation method uses optical density (OD) to monitor bacterial growth (or lack thereof) in $10 \mu 1$ micro-reactors. It is thus a microfluidic analog of the classic microdilution method, with the added advantage of a shorter assay time. However, like the commercially available micro-dilution instruments/methods, it, along with the other emerging techniques mentioned above, is not able to distinguish between the bactericidal and bacteriostatic action of the antibiotics. In the present work, we demonstrate a method that is not only generic (like those using turbidity and fluorescence), but also significantly faster and capable of providing information regarding the mode of action of the antibiotic.

\subsection{Theory and Calculations}

At the core of our method lies an electrical technique that tracks the number of live bacteria in suspensions (Puttaswamy et al. 2011; Puttaswamy and Sengupta 2010a). Our method utilizes the ability of viable bacterial cells to become "polarized" in the presence of the AC electric field. This polarization leads to a buildup of charges across the intact 
membrane of viable bacterial cells (Asami 2002) and hence these cells effectively behave like electrical capacitors. As the viable bacteria reproduce in a suspension, greater numbers of bacteria result in an increase in the charge stored in the interior of a suspension (the "Bulk" or "Medium" Capacitance $\left(\mathrm{C}_{\mathrm{b}}\right)$ "). This principle has been used to determine the presence of viable bacteria in blood cultures and food samples (Puttaswamy et al. 2011; Puttaswamy and Sengupta 2010a). Moreover, as cell death is accompanied by loss of membrane potential and electrical polarization (Gasol 2008), the death of some cells in the suspension being monitored leads to a measurable decrease in the value of its $C_{b}$. In our prior work (Puttaswamy and Sengupta 2010a), we also observed that $\mathrm{C}_{\mathrm{b}}$ values closely track the bacterial concentration in the sample, rising and falling in concert with the latter. By measuring the electrical Impedance $(\mathrm{Z})$ of the bacterial suspension over a wide range of frequencies (from $1 \mathrm{kHz}$ to $100 \mathrm{MHz}$ ) using an Impedance Analyzer, $\mathrm{C}_{\mathrm{b}}$ can be calculated at each time point.

For this study, our hypothesis was the following: For suspensions containing cells of a particular bacterial strain and antibiotic at a known concentration, if the bacteria are able to grow, then the suspension $\mathrm{C}_{\mathrm{b}}$ will increase with time, whereas if the bacterial cells die, the value of $C_{b}$ will decrease over time. Further, if the bacterial cells are not actively growing or dying, then the value of $\mathrm{C}_{\mathrm{b}}$ will remain essentially unchanged. This would occur because the measured $\mathrm{C}_{\mathrm{b}}$ of the suspension at any given point in time is the sum of the bulk capacitance of the solution in which the bacteria are suspended $\left(\mathrm{C}_{\mathrm{b}_{-} \text {soln }}\right)$ and that of the viable bacteria in suspension. The number of viable bacteria in the system (n) is given by

$$
n=n_{o} e^{k t}
$$


where $\mathrm{n}_{0}$ is the initial number of bacteria present and $\mathrm{k}$ is the specific growth/death rate (positive for systems in which the cell number is increasing and negative for those in which the cell number is decreasing). Thus, the value of the bulk capacitance of the suspension, as a function of time, would be given by

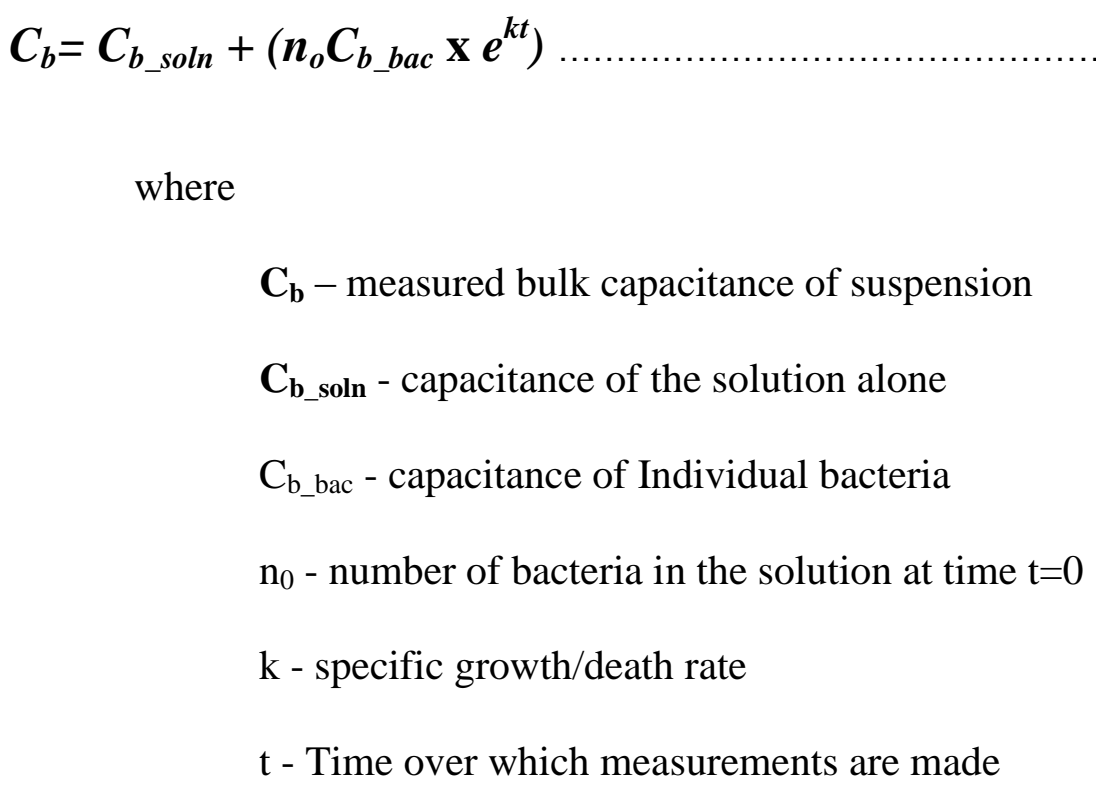

Thus, in theory, if a set of $\mathrm{C}_{\mathrm{b}}$ values (as a function of time) is fit to Equation (7.2) above, and various parameters estimated, then the value of $\mathrm{k}$ will be positive for systems with bacterial proliferation, negative for systems in which bacteria are dying off, and be (statistically) equal to zero for systems in which bacteria are prevented from reproducing, but are not being killed. Hence this method, when applied to samples with different concentrations of antibiotics, can be used to not only determine Minimum Inhibitory Concentrations (MICs), but also to distinguish between bacteriostatic and bactericidal action of antibiotics. 
This method of detection of bacteria in suspension is different from many of the current methods that use electrical measurements to detect bacterial proliferation (Eden and Eden 1984; Felice et al. 1999; Firstenberg-Eden and Zindulis 1984; Yang and Bashir 2008). Many of these methods are based on detecting changes in the overall electrical resistance/impedance (solution conductivity) brought about by bacterial metabolism. In contrast, our method actually tracks the increase in the number of bacteria which is in direct correlation with increase in $\mathrm{C}_{\mathrm{b}}$ and by using this we are able to detect bacterial presence in a given sample much faster.

\subsection{Materials and Methods}

\section{$\underline{7.4 .1}$ Bacterial cultures}

Escherichia coli (ATCC 25922), Staphylococcus aureus (ATCC 29213) and Pseudomonas aeruginosa (ATCC 27853) (bacterial strains whose susceptibility to various antibiotics have been extensively studied (Darrell and Waterworth 1967; De Oliveira et al. 2000; Fass and Barnishan 1979; Locke et al. 2009; Watanakunakorn 1983; Wiegand et al. 2008) are cultured for 12-48 hrs in Tryptic Soy Broth (TSB) to obtain log cultures. These strains were specifically selected as they are 3 of the commonly used control ATCC strains for AST studies (Wiegand et al. 2008).

\subsection{2 $\underline{\text { Sample preparation }}$}

The cultures are centrifuged, and the pellets (bacterial cells) re-suspended in TSB. The OD of these samples is measured against the control (TSB without bacteria) using a 
UV-Vis spectrophotometer (UV 1650PC, Shimadzu Scientific Inc) at $625 \mathrm{~nm}$ and the samples are adjusted by diluting with TSB to have an OD in the $0.08-0.13$ range. These samples, assumed to have a bacterial concentration of $\sim 10^{8} \mathrm{CFU} / \mathrm{ml}$, are then serially diluted in sterile Mueller Hinton (MH) broth to obtain a bacterial concentration of $10^{6}$ $\mathrm{CFU} / \mathrm{ml}$. To the latter, equal volumes of antibiotic solutions in $\mathrm{MH}$ broth are added to obtain bacterial concentrations of $\sim 5 \times 10^{5} \mathrm{CFU} / \mathrm{ml}$ (the recommended inoculation concentration for the broth macrodilution AST). The antibiotic dilutions used in the experiments are prepared in sterile $\mathrm{MH}$ broth at twice the concentrations of the desired final antibiotic concentrations, as the solution will be inoculated with equal amounts of $10^{6} \mathrm{CFU} / \mathrm{ml}$ bacterial solutions. Five milliliters each of the $10^{6} \mathrm{CFU} / \mathrm{ml}$ bacterial samples are added to $5 \mathrm{ml}$ of prepared antibiotic solutions to give a final bacterial concentration of $\sim 5 \mathrm{X} 10^{5} \mathrm{CFU} / \mathrm{ml}$ in each tube with antibiotic concentrations of $0.5 \mathrm{mg} / \mathrm{l}, 1 \mathrm{mg} / \mathrm{l}, 2 \mathrm{mg} / \mathrm{l}$, $4 \mathrm{mg} / 1,8 \mathrm{mg} / 1,16 \mathrm{mg} / 1,32 \mathrm{mg} / 1,64 \mathrm{mg} / 1,128 \mathrm{mg} / 1$. The combination of bacteria-antibiotic pairs used in the experiments is given in Table 7.2. With each bacterium-antibiotic pair, a control sample is also incubated with $5 \mathrm{X} 10^{5} \mathrm{CFU} / \mathrm{ml}$ bacterial concentrations but without the antibiotic. These tubes are incubated on a shaking platform (Fisher Scientific Nutating Mixer) within an incubator ((Fisher 637D Isotemp Incubator) at $37^{\circ} \mathrm{C}$ for 4 hours.

\subsubsection{Multi-frequency Impedance Measurements}

Every hour, we drew a small $(250 \mu \mathrm{l})$ aliquot from the culture, then estimate the Bulk Capacitance of the sample using previously described protocols (Ahmad et al. 2011; Puttaswamy and Sengupta 2010a). Briefly, the aliquot is injected into the microfluidic 
cassette (Figure 7.1c). The gold electrodes on the cassette are connected via a $16047 \mathrm{E}$ connector, an Agilent 4294A Precision Impedance Analyzer (Agilent Technologies) and electrical impedance measurements are taken using a $500 \mathrm{mV}$ AC source over a frequency range of $1 \mathrm{kHz}$ to $100 \mathrm{MHz}$ (200 logarithmically equi-spaced frequency points). Parallel $100 \mu 1$ samples are also plated out (after appropriate serial dilution) at the same point in time to verify bacterial concentration (not shown). Electrical Impedance data obtained from the analyzer is in the form of Resistance (R) and Reactance (X) values at 200 frequencies $(\omega)$ between $1 \mathrm{kHz}$ and $100 \mathrm{MHz}$.

\subsubsection{Data Analysis}

The data obtained from the Impedance analyzer is analyzed using $\mathrm{ZView}^{\mathrm{TM}}$ (commercially available software written for use in Electrical/Electrochemical Impedance Spectroscopy). This software fits the raw data ( $\mathrm{R}$ and $\mathrm{X}$ as a function of $\omega$ ) obtained from the impedance analyzer to the proposed electrical circuit for the suspension (Figure 7.1b) (Sengupta et al. 2006a; Sengupta et al. 2005b). The software provides best estimates for each of the components of the circuit: the electrode resistance $\left(R_{e}\right)$, the lead inductance $\left(\mathrm{L}_{\mathrm{e}}\right)$, the bulk solution resistance $\left(\mathrm{R}_{\mathrm{b}}\right)$, the electro-chemical interfacial capacitance $\left(\mathrm{C}_{\mathrm{e}}\right)$, and bulk capacitance (CPE) along with a confidence interval for these parameters. Values of $\mathrm{C}_{\mathrm{b}}$ obtained at various points in time are recorded for each suspension (bacteria of interest at a given antibiotic concentration). By providing estimates of each individual parameter, the software allows the user to distinguish changes to the overall impedance occurring due to an increase in the bulk capacitance (bacterial number), from other 
factors, such as changes to the bulk resistance (solution conductivity) due to temperature changes.

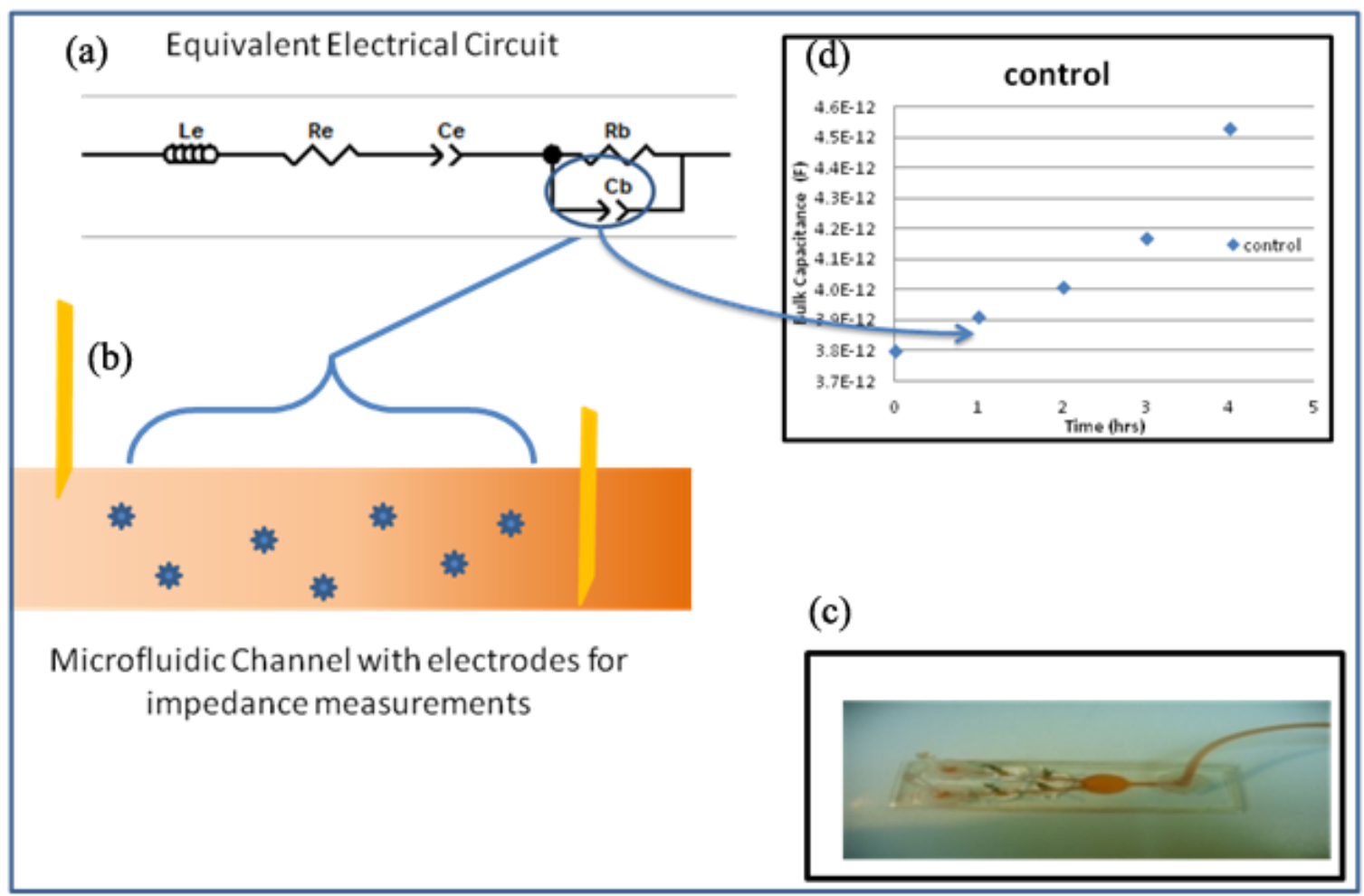

Figure 7.1: Microfluidic channel design and equivalent circuit

(a) Equivalent circuit used to model the electrical behavior of suspensions of bacteria in contact with metal electrodes. Prior to taking electrical measurements, the bacteria suspensions (along with antibiotics) are loaded in micro-channels, shown schematically in (b) and pictured in (c). The electrical measurements are analyzed to obtain the value of the bulk capacitance $(\mathrm{Cb})$, which is then monitored as a function of time (as in (d)).

\subsection{Results and Discussion}

In Figure 7.2, we show (for selected samples) how the $C_{b}$ changes over time for various suspensions. Since the $C_{b}$ at time $t=0$ is different for individual samples (due to differences in the exact number of live bacteria present), we plot the difference from the initial value $\left[\Delta \mathrm{C}_{\mathrm{b}}(\mathrm{t})=\mathrm{C}_{\mathrm{b}}(\mathrm{t})-\mathrm{C}_{\mathrm{b}}(\mathrm{t}=0)\right]$ to aid in visualization of the changes. For all 
bacteria-antibiotic pairs, the $C_{b}$ values increase monotonically with time when the concentration of the antibiotic is less than the MIC. At or above the MIC, however, the $C_{b}$ values either begin to fall $\left(\Delta C_{b}\right.$ negative), or remain unchanged $\left(\Delta C_{b} \cong 0\right)$, depending on whether the antibiotic exerts a bactericidal or bacteriostatic effect, respectively. In some cases, as in the data shown in Figure 7.2(a) and (b) (E. coli), the differences between growth, stasis, and cell-death are clearly discernible from the plot. In some other cases, such as in Figure 7.2(e) and (f) (S. aureus), the differences are not as obvious.

To draw more objective inferences from the data, the recorded values of $\mathrm{C}_{\mathrm{b}}$ at different points in time (t) are fit to the mathematical model mentioned in Equation (7.2) using the software $\mathrm{R}^{\mathrm{TM}}$. The output from the software gives its best estimate for variables in Equation (7.2), including that for K (specific growth rate) and the "P-value" for the estimate. These values are displayed in Table 7.1 for all antibiotic-bacteria pairs tested. 

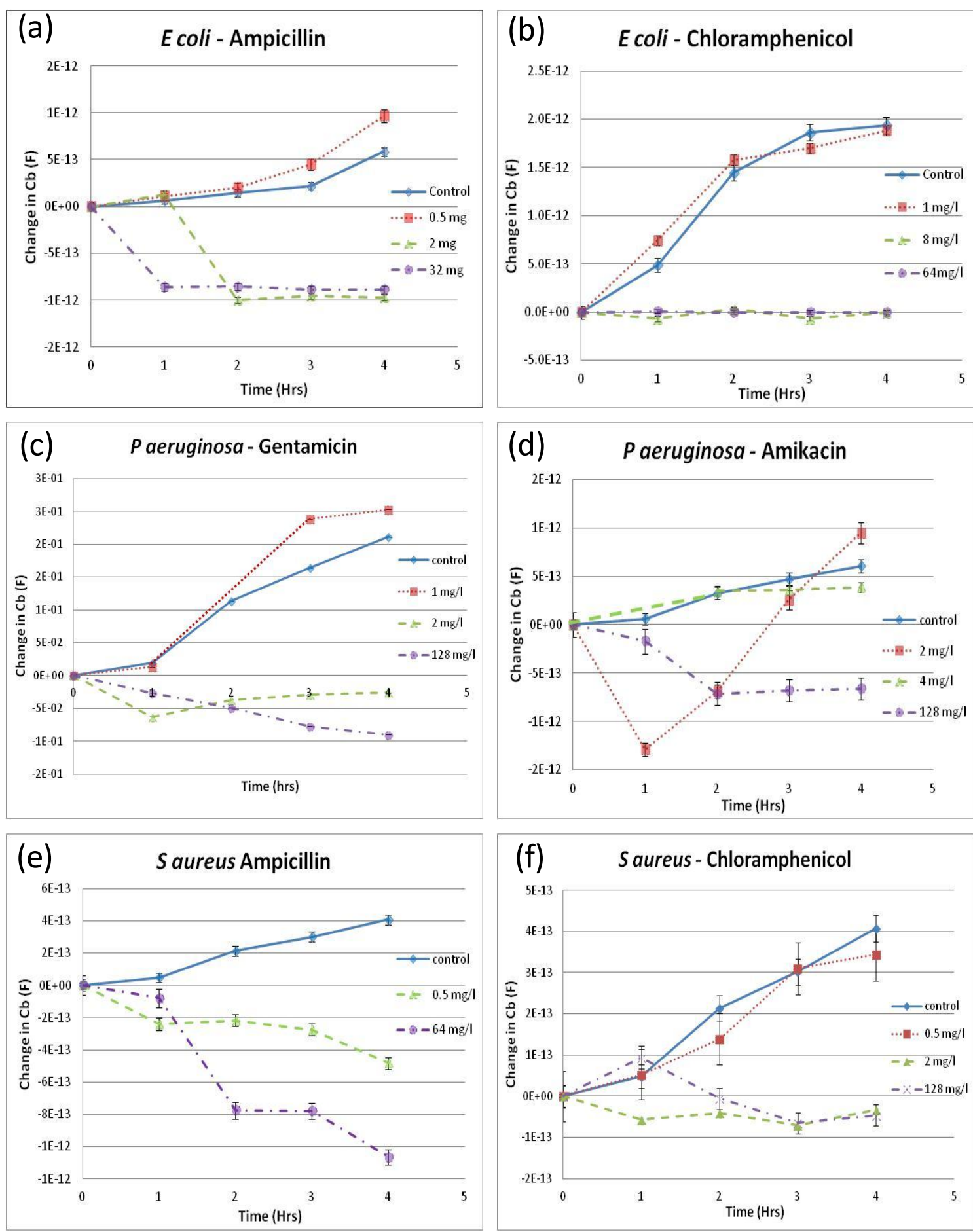

Figure 7.2: Antibiotic susceptibility plots for bacteria in antibiotics

Plots showing how the value of the bulk capacitance of bacterial suspensions change over time, for three bacterial strains exposed to different concentrations known bactericidal (left) and bacteriostatic (right) antibiotic (Only selected concentrations of antibiotics shown). 
The $\mathrm{K}$-value indicates whether the bacteria in the sample are proliferating $(K>0)$, dying $(\mathrm{K}<0)$, or static $(\mathrm{K}=0)$. The accompanying $\mathrm{P}$-value indicates the probability that the true value of $\mathrm{K}$ is zero, and has a value between 0 and $1 . \mathrm{P}$ value allows a decision about the rejection or maintenance of the null hypothesis which in this case is that the $\mathrm{K}$ value (bacterial growth rate) is zero. If the $\mathrm{P}$ value is greater than 0.9 for a given sample set, then the value of $\mathrm{K}$ is considered to be zero. Thus, a sample with a positive $\mathrm{K}$ and $\mathrm{P}$ value $<0.9$ has bacteria whose numbers are exponentially increasing, while those with a negative $\mathrm{K}$ and a P-value of $<0.9$ has bacteria that are being killed (Bactericidal effect). However, irrespective of the value of $\mathrm{K}$, if the P-value is $>0.9$, this is taken to indicate that there is no change in the number of live bacteria in the sample (Bacteriostatic effect). Using this criterion to determine growth, death or stasis, the minimum concentration of antibiotic, for which the value of $\mathrm{K}$ becomes statistically zero (or clearly negative) is the MIC. As listed in Table 7.2, in all cases, MIC values obtained using our method fall within the expected range for the chosen antibiotic-bacteria combination. Also, for systems where the action of the antibiotic is known to be bacteriostatic (Amikacin for $P$. aeruginosa; Chloramphenicol for E. coli and $S$. aureus) the P-value remains $>0.9$ for all concentrations of antibiotic greater than the MIC. In contrast, at concentrations >MIC of the known bactericidal drugs (Ampicillin and Gentamicin), not only is the value of K negative, but the $\mathrm{P}$-value is $<0.9$, indicating cell die-off. 


\begin{tabular}{|c|c|c|c|c|c|c|}
\hline \multirow{3}{*}{$\begin{array}{l}\text { Antibiotic } \\
\text { Concentr } \\
\text { ation } \\
(\mathrm{mg} / \mathrm{l})\end{array}$} & \multicolumn{6}{|c|}{$\begin{array}{l}\text { Value of } K \text { from eqn.(2) calculated using } R^{\mathrm{TM}} \text { software, with P-value in } \\
\text { parentheses }\end{array}$} \\
\hline & \multicolumn{2}{|c|}{$\begin{array}{l}\text { Escherichia coli } \\
\text { ATCC } 25922\end{array}$} & \multicolumn{2}{|c|}{$\begin{array}{l}\text { Pseudomonas aeruginosa } \\
\text { ATCC } 27853\end{array}$} & \multicolumn{2}{|c|}{$\begin{array}{l}\text { Staphylococcus aureus } \\
\text { ATCC } 29213\end{array}$} \\
\hline & Ampicillin & $\begin{array}{l}\text { Chloram- } \\
\text { phenicol }\end{array}$ & Gentamicin & Amikacin & Ampicillin & $\begin{array}{l}\text { Chloram- } \\
\text { phenicol }\end{array}$ \\
\hline Control & $\begin{array}{l}0.53876 \\
(0.035)\end{array}$ & $\begin{array}{l}0.4479 \\
(0.6735)\end{array}$ & $\begin{array}{l}0.04821 \\
(0.819)\end{array}$ & $\begin{array}{l}0.13 \\
(0.756)\end{array}$ & $\begin{array}{l}0.03685 \\
(0.839)\end{array}$ & $\begin{array}{l}0.2772 \\
(0.0648)\end{array}$ \\
\hline 0.5 & $\begin{array}{l}0.692 \\
(0.368)\end{array}$ & $\begin{array}{l}0.56079 \\
(0.856)\end{array}$ & $\begin{array}{l}0.06857 \\
(0.819)\end{array}$ & $\begin{array}{l}1.73 \\
(0.43)\end{array}$ & $\begin{array}{l}-0.026 \\
(0.878)\end{array}$ & $\begin{array}{l}0.4332 \\
(0.849)\end{array}$ \\
\hline 1 & $\begin{array}{l}0.59 \\
(0.368)\end{array}$ & $\begin{array}{l}0.2664 \\
(0.75)\end{array}$ & $\begin{array}{l}0.5607 \\
(0.639)\end{array}$ & $\begin{array}{l}0.2525 \\
(0.539)\end{array}$ & $\begin{array}{l}-0.4457 \\
(0.1673)\end{array}$ & $\begin{array}{l}1.42 \\
(0.707)\end{array}$ \\
\hline 2 & $\begin{array}{l}-0.278 \\
(0.75)\end{array}$ & $\begin{array}{l}0.0806 \\
(0.794)\end{array}$ & $\begin{array}{l}0.15 \\
(0.971)\end{array}$ & $\begin{array}{l}0.792 \\
(0.62)\end{array}$ & $\begin{array}{l}-0.53 \\
(0.26)\end{array}$ & $\begin{array}{l}0.671 \\
(0.96)\end{array}$ \\
\hline 4 & $\begin{array}{l}-0.898 \\
(0.2285)\end{array}$ & $\begin{array}{l}0.227 \\
(0.628)\end{array}$ & $\begin{array}{l}-0.7194 \\
(0.55)\end{array}$ & $\begin{array}{l}-0.0013 \\
(1)\end{array}$ & $\begin{array}{l}-0.3969 \\
(0.415)\end{array}$ & $\begin{array}{l}0.0268 \\
(0.95)\end{array}$ \\
\hline 8 & $\begin{array}{l}-1.23 \\
(0.36)\end{array}$ & $\begin{array}{l}0.209 \\
(0.941)\end{array}$ & $\begin{array}{l}-0.6121 \\
(0.7918)\end{array}$ & $\begin{array}{l}-0.7373 \\
(0.995)\end{array}$ & $\begin{array}{l}-0.568 \\
(0.191)\end{array}$ & $\begin{array}{l}-0.632 \\
(0.944)\end{array}$ \\
\hline 16 & $\begin{array}{l}-0.511 \\
(0.66)\end{array}$ & $\begin{array}{l}-0.04 \\
(0.945)\end{array}$ & $\begin{array}{l}-1.0139 \\
(0.495)\end{array}$ & $\begin{array}{l}-0.065 \\
(0.995)\end{array}$ & $\begin{array}{l}-0.2478 \\
(0.609)\end{array}$ & $\begin{array}{l}-0.1003 \\
(0.94)\end{array}$ \\
\hline 32 & $\begin{array}{l}-3.54 \\
(0.064)\end{array}$ & $\begin{array}{l}-0.0089 \\
(0.989)\end{array}$ & $\begin{array}{l}-0.1791 \\
(0.6486)\end{array}$ & $\begin{array}{l}-0.0069 \\
(0.90)\end{array}$ & $\begin{array}{l}-0.4627 \\
(0.5916)\end{array}$ & $\begin{array}{l}0.05014 \\
(0.967)\end{array}$ \\
\hline 64 & $\begin{array}{l}-10.35 \\
(0.083)\end{array}$ & $\begin{array}{l}-0.0119 \\
(0.995)\end{array}$ & $\begin{array}{l}-0.152 \\
(0.827)\end{array}$ & $\begin{array}{l}-0.38 \\
(0.922)\end{array}$ & $\begin{array}{l}-0.291 \\
(0.174)\end{array}$ & $\begin{array}{l}-0.1814 \\
(0.96)\end{array}$ \\
\hline 128 & $\begin{array}{l}-2.35 \\
(0.6083)\end{array}$ & $\begin{array}{l}0.024 \\
(0.994)\end{array}$ & $\begin{array}{l}-0.5723 \\
(0.1889)\end{array}$ & $\begin{array}{l}-0.04121 \\
(0.987)\end{array}$ & $\begin{array}{l}-2.25 \\
(0.189)\end{array}$ & $\begin{array}{l}-0.02605 \\
(0.982)\end{array}$ \\
\hline
\end{tabular}

Table 7.1: Antibiotic susceptibility statistical analysis using $R^{T M}$

Value of $K$ from equation (2) calculated using $R^{T M}$ software, with P-value in parentheses. The values of $K$ obtained by fitting the along with the recorded values of bulk capacitance $(\mathrm{Cb})$ at different points in time ( $t$ ) to Equation (2), using the Software $R^{T M}$ (with P-values, also calculated by $R$, in parenthesis) for all samples tested (each with a different concentration of antibiotic). The boxes corresponding to the MIC value for each antibiotic-bacteria pair, as determined by criteria described in the text, is marked with thick borders. The shaded areas indicate the expected MIC range for a given antibiotic-bacteria pair. 


\begin{tabular}{llll}
\hline Bacteria & Antibiotic & $\begin{array}{l}\text { Known } \\
\text { MIC } \\
\text { (mg/l) }\end{array}$ & $\begin{array}{l}\text { Our MIC } \\
\text { (mg/l) }\end{array}$ \\
\hline $\begin{array}{l}\text { Escherichia coli } \\
\text { (ATCC 25922) }\end{array}$ & $\begin{array}{l}\text { Ampicllin } \\
\text { (Bactericidal) }\end{array}$ & $2-8$ & 2 \\
Chloramphenicol & $2-8$ & 8 \\
(Bacteriostatic) & $1-4$ & 1 \\
$\begin{array}{l}\text { Psuedomonas } \\
\text { aeruginosa } \\
\text { (ATCC 27853) }\end{array}$ & $\begin{array}{l}\text { Gentamicin } \\
\text { (Bactericidal) }\end{array}$ & $\begin{array}{l}\text { Amikacin } \\
\text { (Bacteriostatic) }\end{array}$ & $2-8$ \\
$\begin{array}{l}\text { Staphylococcus } \\
\text { aureus }\end{array}$ & $\begin{array}{l}\text { Ampicillin } \\
\text { (Bactericidal) }\end{array}$ & $0.5-2$ & 4 \\
(ATCC 29213) & $\begin{array}{l}\text { Chloramphenicol } \\
\text { (Bacteriostatic) }\end{array}$ & $2-8$ & 2 \\
\hline
\end{tabular}

Table 7.2: MIC value comparison by our method vs. Standard values

Comparison of MIC values obtained using our method to known MIC values for all combinations of antibiotic and bacterial strains tested.

\subsection{Conclusions}

To conclude, we would like to highlight that in this work, we have demonstrated the feasibility of using our novel electrical method to not only determine the MIC of candidate antibiotics to bacterial strains of interest in 4 hours, but also to infer the effect of the antibiotic on the strain (whether bactericidal or bacteriostatic) simultaneously. The technique needs to be refined and made more user-friendly (by automating the aliquot collection, impedance measurement and data analysis), and also tested extensively against a wider panel of antibiotics and strains before it can actually be tested in a clinical setting. In fact, once automated and made user-friendly, the described method can also be 
extended to obtain other relevant clinical information as well. For instance, by obtaining electrical measurements at more frequent intervals, it could become possible to conduct time-kill studies in real-time, thereby indicating which antibiotic is able to kill the infectious bacteria faster. Thus, the work reported here represents "proof-of-principle" for a method that can, in the future, be used to rapidly obtain not only MICs, but also other information regarding the antibacterial mode of action. 


\section{CHAPTER 8}

\section{MULTI-FREQUENCY ELECTRICAL IMPEDANCE METHOD FOR THE RAPID DETECTION OF VIABLE MICROORGANISMS, AND THEIR RAPID QUANTIFICATION}

The material presented in this chapter has is currently under revision as "Multi-Frequency Electrical Impedance Method for the Rapid Detection of Viable Microorganisms, and Their Rapid Quantification"

Sachidevi Puttaswamy, Byung-Doo Lee, Ashley Jurgenmeyer and Shramik Sengupta in Analyst

\subsection{Abstract}

Existing culture-based instruments for detecting/quantifying viable bacteria in suspensions (BACTECTM, BacT/Alert ${ }^{\mathrm{TM}}$, Bactometer $^{\mathrm{TM}}$, RABIT $^{\mathrm{TM}}$ etc.) do so based on changes brought about by bacterial metabolism to the physical/chemical properties $\left(\mathrm{O}_{2} / \mathrm{CO}_{2}\right.$ levels, $\mathrm{pH}$, solution-conductivity, etc. $)$ of the medium in which they are suspended. Given the limited metabolic rate of individual bacterial cells, they have a "threshold concentration" of $\sim 10^{7}-10^{8} \mathrm{CFU} / \mathrm{ml}$, and Times to Detection (TTDs) of 12 hours, or longer for low initial loads $(<100 \mathrm{CFU} / \mathrm{ml})$. We have recently developed a method that tracks microbial proliferation in suspensions by monitoring the degree of cell 
polarization of live microbial cells. In an AC electric field, there occurs a build-up of charge at the membrane, causing the cells to act like capacitors. As the microorganisms multiply, there occurs a corresponding increase in the charges thus stored in the interior of the suspension (its "bulk capacitance"), and this increase in bulk capacitance serves as our "signature" indicating the presence of live microorganisms. In this study, we (a) explain the theory underlying our approach; (b) establish that this method is applicable to a variety of viable microorganisms (aerobic bacteria, anaerobic bacteria, yeasts, and molds), showing that the "Threshold Concentration" $\left(\mathrm{n}_{\mathrm{T}}\right)$ for their detection is $\sim 10^{3}-10^{4}$ CFU/ml, and the TTDs are a function of the initial load $\left(\mathrm{n}_{0}\right)$ and doubling time $\left(\mathrm{t}_{\mathrm{D}}\right)$ of the microorganism $\mathrm{TTD}=1.443 * \mathrm{t}_{\mathrm{D}} * \ln \left(\mathrm{n}_{\mathrm{T}} / \mathrm{n}_{0}\right)$; and (c) show that the method can also be adapted to obtain the "Most Probable Number" (MPN) of coliforms within 6 hrs (vs. $>24$ hrs for existing methods).

\subsection{Introduction}

In the recent past, there have been many advances in detection technologies that make it possible to detect, identify and/or quantify pathogens present in clinical, food, environmental and other samples (Byrne et al. 2009; Sengupta et al. 2006a). Some of these, including commercially available products like PNA-FISH®, GeneXpert@, BioMark $^{\mathrm{TM}}$ etc, detect/identify/quantify bacteria by targeting specific DNA sequences, amplifying the targets using Polymerase Chain Reaction (PCR) and detecting/identifying/quantifying the PCR-products by optical, electrical or other means. Other technologies, such as SPREETA ${ }^{\mathrm{TM}}$, ProteOn XPR36 ${ }^{\mathrm{TM}}$, SensiQ $^{\mathrm{TM}}$, Biacore ${ }^{\mathrm{TM}}$ etc. 
use antibodies to tag or sequester the target cells for detection, identification or quantification. While technologies that use these two approaches are useful in a number of situations (such as PNA-FISH® being used to identify bacteria present in positive blood cultures, and Biacore ${ }^{\mathrm{TM}}, \mathrm{SensiQ}^{\mathrm{TM}}$, etc. which is used for pathogen monitoring), there remains a class of "real world" situations where these technologies do not work. In these situations, one seeks to determine whether there are any viable (live) microorganisms present in a sample with the understanding that, even if present, their concentrations will be very low (100 CFU/ml or lower), and that it is very likely that the sample also contains a larger number of dead microorganisms.

In some cases, such as in assaying process water used for manufacturing pharmaceuticals (2004), one needs to conduct tests to verify that there are absolutely no live microorganisms remaining in the entire batch after the treatment processes are adopted. The goal of such tests is to detect any surviving microorganism(s). In other cases, the number of live microorganisms remaining must be quantified. For instance, in the case of food quality testing, the US Department of Agriculture (USDA) requires ground beef being shipped to consumers (after processing to kill microorganisms) to contain < 1 CFU of viable E. coli per $25 \mathrm{~g}$ of beef (Johnson et al. 1998) and the US Pasteurized Milk Ordinance requires "Grade A" pasteurized milk to have a total viable bacterial count of $\leq 20,000 \mathrm{CFU} / \mathrm{ml}$ and a coliform count of $\leq 10 \mathrm{CFU} / \mathrm{ml}$ (Hayes and Boor 2001). Here, testing is conducted to find the (small) number of surviving microorganisms amongst the many killed as a result of procedures such as pasteurization. Similarly, in the case of water quality monitoring, the Environmental Protection Agency (EPA) specifies that concentration of viable coliform bacteria should be $<5 \mathrm{CFU} / \mathrm{ml}$ for 
primary contact recreational waters (such as lakes and beaches) and $8 \mathrm{CFU} / \mathrm{ml}$ for secondary contact recreational waters (such as streams) (Anderson and Davidson 1997). Once again, testing is conducted to detect and quantify small numbers of viable microorganisms in the possible presence of larger numbers of dead microorganisms.

In these, and related situations, the technologies used continue to be "culture"based. Here, to detect the presence of viable microorganisms, samples are added to a known volume of sterile microbial growth media. If present, viable microorganisms metabolize, consuming glucose and oxygen, and releasing lactic/pyruvic acid, $\mathrm{CO}_{2}$, etc. The metabolic activity thus leads to changes in the properties of the surrounding medium such as a decrease in $\mathrm{pH}$, an increase in conductivity, or a change in levels of $\mathrm{O}_{2}$ or $\mathrm{CO}_{2}$. If such changes are detected, they can be taken to indicate the presence of viable microorganisms in the sample added to the growth media. This approach forms the basis of a large number of commercial products such as the BACTEC ${ }^{\mathrm{TM}}$ and BacT/Alert ${ }^{\mathrm{TM}}$ that detect changes in $\mathrm{CO}_{2}$ levels (Horvath et al. 2004; Thorpe et al. 1990), the Difco-ESP System $^{\mathrm{TM}}$ that monitors pressure changes due to gas consumption or production (Woods et al. 1997), and the Bactometer ${ }^{\mathrm{TM}}$ and $\mathrm{RABIT}^{\mathrm{TM}}$ that monitor the conductivity of the growth medium(Bolton 1990; Russell 2000). These instruments work very well for a wide variety of applications because the presence of dead microorganisms does not affect the results, and that the theoretical Limit of Detection (LOD) is 1 CFU. They are also conducive to automation, keeping the costs low, and thus leading to their popularity.

These instruments, however, suffer from one major drawback: the time taken to detect the presence of living microorganisms is considerable (hours to days), especially when initial loads present are low $(<\sim 100 \mathrm{CFU} / \mathrm{ml}$ ) (Sengupta et al. 2010). This is 
because the detection can be done only when the cumulative effect of microbial metabolism (and proliferation) changes the quantity that they monitor $\left(\mathrm{O}_{2} / \mathrm{CO}_{2}\right.$ levels, $\mathrm{pH}$, conductivity, etc.) by a measureable degree. But the rate of metabolism of individual cells is inherently limited. For instance, even a relatively fast growing/metabolizing bacterium like $E$ coli consumes only $2 \times 10^{-14}$ moles of $\mathrm{O}_{2}$ per hour (Sengupta et al. 2006a), whereas a typical well-oxygenated suspension has a dissolved $\mathrm{O}_{2}$ concentration $10^{-6}$ moles/ml (Körner and Zumft 1989). Hence, with the current systems, one has to wait for the concentration of living microorganisms to reach a "threshold" of $\sim 10^{6}$ to $10^{8}$ $/ \mathrm{ml}$ before their metabolism can change the medium properties $\left(\mathrm{O}_{2} / \mathrm{CO}_{2}\right.$ concentration, $\mathrm{pH}$, conductivity etc) to a measurable degree (Smith et al. 2008). The time that elapses before a change in the medium properties is measured (and the presence of live microorganisms in the original culture inferred), is referred to as the Time to Detection (TTD). The TTD typically depends on the initial load of viable microorganisms in the sample and the metabolic rate of these microorganisms. In general, TTDs are longer for samples with lower initial loads and for microbes with slower metabolic rates (longer doubling times). The extremely limited amount of metabolites generated/consumed also limits how much TTDs can be reduced by improving the performance of sensors to detect changes in $\mathrm{pH}, \mathrm{O}_{2} / \mathrm{CO}_{2}$ levels, etc. One approach to reducing TTDs that has had some success is the pre-concentration of cells/particles prior to culture, using methods such as filtration (Noble and Weisberg 2005), centrifugation (Noble and Weisberg 2005), immunomagnetic separation (Liu et al. 2004) or dielectrophoresis (DEP) (Yang and Bashir 2008). But not only do these processes themselves take time and require additional resources, their efficiency of capture is often not $100 \%$, leading to potential false 
negatives and/or errors in establishing the count. Moreover, since after pre-concentration, the samples are assayed using the same "slow" culture based methods, the benefits of using pre-concentration is also rather limited.

These long TTDs may result in adverse consequences. In food industries, by the time the counts (most probable numbers or MPNs) of viable bacteria become available (2+ days using current technologies) the product may have already been shipped and if the counts exceeded the approved limits, the product must be recalled. Every year (since 1997), an average of about 4,500 metric tons (about 10 million lbs) of meat and poultry are recalled by US industries (Teratanavat and Hooker 2004) and the USDA Economic Research Service estimates that about 19 million lbs of milk are lost due to spoilage each year (Doyle 2007). For facilities such as campgrounds and beach resorts, water samples are typically collected daily, but the MPN values are available only after 2-3 days. Thus, a particular facility will not be immediately aware of increased risks to users. On the other hand, after the risk has been identified (and actions such as chlorination undertaken), it takes a similarly long time to ascertain that the bacterial counts are back to acceptable levels (and the facility can re-open only after this has been established). The former leads to spread of disease among its users and the latter leads to economic losses. In 2008, the number of closing and advisory days at ocean, bay and Great Lakes beaches topped 20,000 for the $4^{\text {th }}$ consecutive year (Dorfman et al. 2009). Thus, there remains a compelling "real world" need for a method that can rapidly detect and/or estimate the numbers of low numbers of viable microorganisms (i.e. have greatly reduced TTDs). It would perhaps also be preferred (given the inherent limitations discussed above) that this 
method did not rely on detecting the effects of microbial metabolism. In this work, we describe in detail a method that meets both these criteria.

Briefly, our method relies on the microbial polarizability in the presence of high frequency $\mathrm{AC}$ electric field; there occurs a buildup of charge at the cell-membrane of viable cells, causing these cells to behave like electrical capacitors. The proliferation of any viable microorganism present (an increase in the number of live microbial cells) increases the "bulk capacitance" of the sample under investigation, and this increase in bulk capacitance serves as our "signature" indicating the presence of live microorganisms. Confining the sample to a long narrow channel increases the resistance of the solution, and this amplifies the influence of microbial charge storage on the measured impedance (by increasing the effective RC-time constant). A variant of impedance spectroscopy is used to estimate the charge storage with high sensitivity, and are able to detect microbial proliferation. In our previous work, we reported using this approach to detect E. coli in substrates such as food matrices (milk and apple juice) (Puttaswamy and Sengupta 2010b) and Blood Culture broths (Puttaswamy et al. 2011). In both cases, our "threshold concentrations" (at which we were able to infer the presence of living microorganisms) was $\sim 10^{3}-10^{4} \mathrm{CFU} / \mathrm{ml}$ (compared to $10^{6}-10^{8}$ for current systems), and hence our TTDs were 4-10 times smaller than those obtained using current systems.

In this work, our aim is to (a) explain the theory underlying our approach (b) establish that this method is applicable to a variety of viable microorganisms (aerobic bacteria, anaerobic bacteria, yeasts, and molds), and that the threshold concentrations are similar for different microorganisms (c) investigate how the Time to Detection (TTD) 
varies as a function of the initial load $\left(\mathrm{n}_{0}\right)$ and doubling time $\left(\mathrm{t}_{\mathrm{D}}\right)$ of the microorganism; and (d) show that the method can also be adapted to obtain the "Most Probable Number" (MPN) of viable bacteria of interest.

\subsection{Theory and Calculations}

The software $\mathrm{ZView}{ }^{\mathrm{TM}}$ used for analysis of the data from the Impedance Analyzer is written for use in Electrical/Electrochemical Impedance Spectroscopy. It accepts as input, the measured values of Resistance $(\mathrm{R})$ and Reactance $(\mathrm{X})$ at multiple frequencies, allows the user to propose an equivalent circuit for the material being investigated, and provides an estimate of the values of the individual elements in the equivalent circuit $\left(\mathrm{L}_{\mathrm{e}}, \mathrm{R}_{\mathrm{e}}, \mathrm{C}_{\mathrm{e}}\right.$, $\mathrm{R}_{\mathrm{b}}$, CPE-T, and CPE-P in our case), along with an "error" of the estimate. If we are able to unequivocally establish that, after a certain duration of incubation, the value of the magnitude of the CPE (the CPE-T parameter) has increased (implying that the number of charge-storing species in the suspension's interior has increased), it can be attributed to microbial proliferation (and hence presence of viable microorganisms) in the original sample.

By providing estimates of each individual parameter, the software allows the user to distinguish changes to the overall impedance occurring due to an increase in the bulk capacitance of the suspension on account of microbial proliferation (our phenomenon of interest) from changes in other factors (if any). For instance, small changes in temperature changes would be expected to change the conductivity of the solution significantly (Cussler 1997), which would result in a change in the bulk resistance $\left(\mathrm{R}_{\mathrm{b}}\right)$ 
while leaving the bulk capacitance $\left(\mathrm{C}_{\mathrm{b}}\right)$, which arises from cell-membrane polarization, largely unaffected (Schwan and Foster 1980). Also with time, the electrode may undergo surface modifications as a result of which the properties of the electrode/electrochemical interface $\left(L_{e}, R_{e}\right.$ and $\left.C_{e}\right)$ may change (Baboian 2005). The interfacial capacitance $\left(C_{e}\right)$ may also change due to change in $\mathrm{pH}$ and temperature since the ions in the interfacial "double layer" are in electrochemical equilibrium with the bulk (Guyer et al. 2003). However, our analysis of the $\mathrm{Z}$ vs. $\omega$ data allows us to calculate changes to all parameters, individually. By focusing exclusively on the change in the bulk capacitance, we are able to proceed effectively, despite the occurrence of these other phenomena.

\subsection{Times to Detection (TTDs) Determination}

The Z-View ${ }^{\mathrm{TM}}$ software generates not only an estimate of the parameters (such as the Bulk Capacitance, CPE-T value), but also an "error". For two recorded values to be "significantly" different from each other, there should not be any overlap between the ranges ([value - error] to [value + error]) of the two readings. As we calculate the value of the Bulk Capacitance after various intervals of time, we examined whether or not the present value is "significantly" greater than the initial (0-hour) value. A "significant" increase in the Bulk Capacitance value would indicate an increase in the number of species capable of storing charge that are dispersed in the medium. Since other such species (such as proteins and blood cells in the case of blood culture, milk proteins and fruit pulp in food substrates, silica particles in environmental samples, etc.) are not expected to increase in number, such an increase is mainly brought about by microbial 
proliferation, as is hence taken as a signature of the presence of viable microorganisms in the sample. The time taken to obtain such a "significant" increase in the value of the Bulk Capacitance is our Time to Detection (TTD). The TTD is a function of the initial load, doubling time and the "threshold concentration", and is given by the equation:

$$
\mathrm{TTD}=1.443 \mathrm{t}_{\mathrm{D}} \ln \left(\mathrm{n}_{\mathrm{T}} / \mathrm{n}_{0}\right)
$$

where

$t_{D}$ is the doubling time of the microorganisms

$\mathrm{n}_{0}$ is the initial concentration, and

$\mathrm{n}_{\mathrm{T}}$ is the threshold concentration

Threshold concentration is defined as the concentration of viable microorganisms in the sample at the point in time that our method can unequivocally discern a change in the bulk capacitance from its baseline $(t=0)$ value. It thus also depends on the magnitude of the baseline value (and hence the composition) of the suspension being assayed. A suspension with a higher baseline bulk capacitance will tend to have a higher threshold concentration. Consider two samples with baseline bulk capacitances of 2 and $10 \mathrm{pF}$, respectively. Assuming that our instrument/data analysis software can discern a 5\% change in either, the proliferating microorganisms would have to contribute a capacitance of $0.1 \mathrm{pF}$ in the first case, and $0.5 \mathrm{pF}$ in the second to bring about these discernible changes. The latter sample would thus need to have more microorganisms generated in 
order for us detect the original viable members, and consequently, it will have a higher threshold concentration.

\subsection{Quantifying the Number of Bacteria Present [Most}

\section{Probable Number (MPN)]}

The "Most Probable Number" (MPN) (Oblinger and Koburger 1975; Sutton February, 2010) is a statistical method used to estimate the concentration of viable bacteria in a sample of interest that is widely used to monitor food and water quality. Briefly, and as depicted schematically in Figure 8.1, it involves serially diluting (by successive factors of 10) a known volume of the sample into bacterial growth media, culturing the "dilution series" bottles obtained for a sufficiently long period of time, and identifying the bottles that are "positive" (where growth of bacteria has occurred). If the sample originally had (say) $500 \mathrm{CFU} / \mathrm{g}$, and we dissolved $1 \mathrm{~g}$ in $1 \mathrm{ml}$ of nutrient media to obtain our first solution (with $500 \mathrm{CFU} / \mathrm{ml}$ ), the subsequent factor-of-ten dilutions should (under ideal conditions) contain 50, and 5 bacteria. A further factor-of-ten dilution will result in a solution with $1 \mathrm{CFU}$ half the time, and $0 \mathrm{CFU}$ on the other occasions (probability of $1 / 2$ that the sample contains one CFU). The probability that the next tenfold dilution will contain $1 \mathrm{CFU}$ is $0.05(5 \%)$. Further dilutions will have even lower probabilities of containing $1 \mathrm{CFU}$. If a given dilution series bottle contains even $1 \mathrm{CFU}$ to begin with, it will ultimately (if cultured for a "sufficiently" long period of time) turn "positive" (display signs of bacterial presence like turbidity). By repeating the dilution experiments a number of times (typically 3 or 5), it is possible to obtain from the number 
of samples turning positive at each level of dilution, a statistical estimate of the number present in the original suspension. These estimates are available in literature as tables.

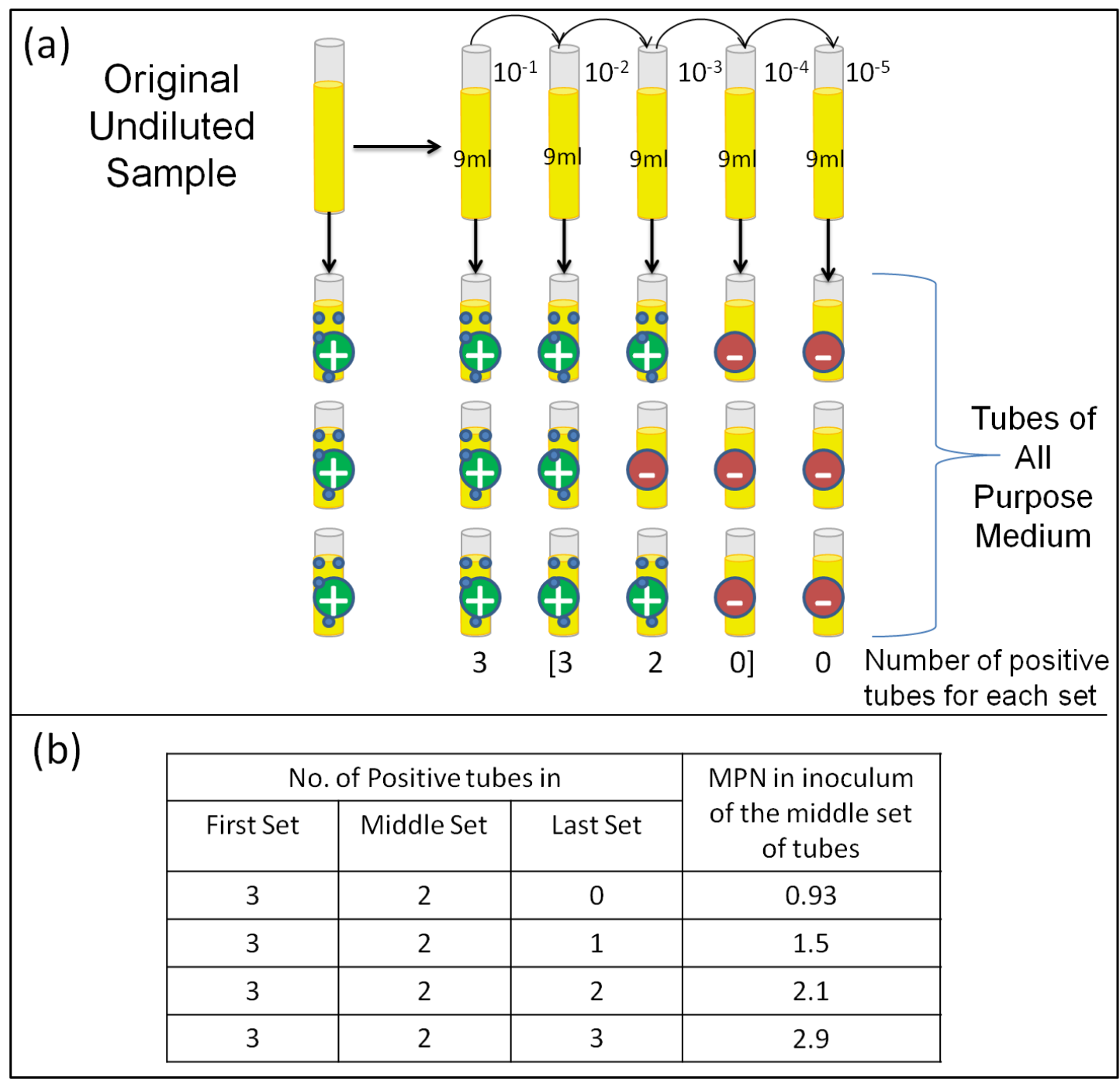

Figure 8.1: Standard MPN method schematics

(a) Schematic of most probable number (MPN) method briefly describing the protocol for the experiment (b) represents an extract from the statistical MPN determination table through which $M P N$ and in turn the actual concentration of bacteria in the original sample can be determined. 
The key to obtaining correct MPNs is to run the culture for a sufficiently long time so that even $1 \mathrm{CFU}$ in the whole suspension (typically $10 \mathrm{ml}$ ) is detected. Using current systems (that typically use turbidity measurements to detect the proliferation of bacteria), bacterial concentrations have to rise to $\sim 10^{7} \mathrm{CFU} / \mathrm{ml}$ (or higher) (Lim 2002) before they can be identified as positive. Our method, as mentioned earlier, identifies samples as "positive" when the calculated value of the Bulk Capacitance increases "significantly" from the baseline $(t=0)$ value of the sample. Because this "significant" change occurs for

filtered growth media when the concentration of bacteria reaches a threshold of $\sim 10^{3}$ CFU/ml (Puttaswamy and Sengupta 2010b), our method can potentially identify positive samples much earlier. Also, samples that do not turn positive before a certain "cutoff time" are typically deemed negative. Since with our method, even one CFU (if present) would be detected much earlier, we can potentially use a cutoff time much shorter than the 24 hrs typically used currently (Wrenn and Venosa 1996). We should hence be able to cut down the time needed to determine the MPN.

\subsection{Experimental Procedures}

\section{$\underline{\text { 8.6.1 }} \underline{\text { Microbial cultures }}$}

Pseudomonas aeruginosa (ATCC 9027) (fast growing aerobic bacteria), Methylobacterium mesophilicum (ATCC 29983) (slow growing aerobic bacteria), Propionibacterium acnes (type III isolate from Millipore) (anaerobic bacteria), Dekkera anomala (ATCC 10559) (yeast), Aspergillus brasiliensis (ATCC 16404) (mold) are used 
in the experiments. A brasiliensis spores were obtained from Microbiologics Inc. in the form of pellets known to contain $\sim 10^{3}$ spores/pellet, and used directly. The other organisms were originally obtained in lyophilized form from ATCC, and log-cultures preserved in glycerol solution within cryotubes prior to use for the experiments described.

\subsubsection{Sample preparation}

The microorganisms in the cryotubes were taken from the freezer $\left(-20^{\circ} \mathrm{C}\right)$ and thawed to reach room temperature. Immediately on thawing, $100 \mu 1$ of the solution in the cryotube was diluted into $900 \mu \mathrm{l}$ of Fluid A $(0.1 \%$ peptone water). These samples were then serially diluted in Fluid A (by appropriate factors, depending on the concentrations of the specific organisms present in the cryotubes) to yield solutions containing $\sim 10^{6}$ $\mathrm{CFU} / \mathrm{ml}$ of the microorganism. One milliliter of this fluid A suspension containing $\sim 10^{6}$ $\mathrm{CFU} / \mathrm{ml}$ of the microorganism was added to $9 \mathrm{ml}$ of appropriate media (as listed in Table 8.1) to yield cultures with initial loads of $\sim 10^{5} \mathrm{CFU} / \mathrm{ml}$. These cultures were incubated at a desired temperature for different amounts of time to obtain "log cultures" with $\sim 10^{8}$ $\mathrm{CFU} / \mathrm{ml}$ of microorganisms. In Table 8.1 , we list the estimated CFU/ml of microorganisms in the cryotubes, the liquid media in which they were subsequently cultured, the temperature of culture, and the time for which they were cultured to obtain $\log$ cultures. These log cultures were then serially diluted in Fluid A and an appropriate volume of the samples containing microorganisms were added to respective sterile media 
(listed in Table 8.1) to yield the "incubation study" media, which were then cultured at conditions mentioned in Table 8.1.

\begin{tabular}{|c|c|c|c|c|c|}
\hline & $\begin{array}{c}\text { M. mesophilicum } \\
\text { ATCC } 29983\end{array}$ & $\begin{array}{c}\text { P. aeruginosa } \\
\text { ATCC } 9027\end{array}$ & $\begin{array}{l}\text { P.acnes } \\
\text { (type III) }\end{array}$ & $\begin{array}{c}\text { D. anomala } \\
\text { ATCC } 10559\end{array}$ & A. brasiliensis \\
\hline $\begin{array}{l}\text { Expected } \\
\text { CFU/ml in } \\
\text { Cryotube }\end{array}$ & $4 \times 10^{7}$ & $2 \times 10^{8}$ & $8 \times 10^{6}$ & $5 \times 10^{6}$ & - \\
\hline $\begin{array}{c}\text { Broth in } \\
\text { which } \\
\text { cultured }\end{array}$ & $\mathrm{R} 2 \mathrm{~A}$ & $\begin{array}{l}\text { Tryptic Soy } \\
\text { Broth }\end{array}$ & $\begin{array}{l}\text { Clear Fluid } \\
\text { thioglycoll } \\
\text { ate medium }\end{array}$ & SAB & SAB \\
\hline $\begin{array}{l}\text { Incubation } \\
\text { condition }\end{array}$ & Standing & standing & Standing & standing & Standing \\
\hline $\begin{array}{l}\text { Incubation } \\
\text { temperature }\end{array}$ & 20 to $25^{\circ} \mathrm{C}$ & 20 to $25^{\circ} \mathrm{C}$ & 30 to $35^{\circ} \mathrm{C}$ & 20 to $25^{\circ} \mathrm{C}$ & 20 to $25^{\circ} \mathrm{C}$ \\
\hline $\begin{array}{c}\text { Time between } \\
\text { Electrical } \\
\text { measurement }\end{array}$ & $6 \mathrm{hrs}$ & $1 \mathrm{hr}$ & $2 \mathrm{hrs}$ & $6 \mathrm{hrs}$ & $6 \mathrm{hrs}$ \\
\hline $\begin{array}{l}\text { Agar used for } \\
\text { plate counts }\end{array}$ & $\mathrm{R} 2 \mathrm{~A}$ & $\begin{array}{c}\text { Tryptic Soy } \\
\text { Agar }\end{array}$ & $\begin{array}{l}\text { Reinforced } \\
\text { Columbia } \\
\text { agar }\end{array}$ & SDA & SDA \\
\hline
\end{tabular}

Table 8.1: Experimental parameters used for testing microorganisms of interest

For $A$ brasiliensis, the pellet (containing $10^{3} \mathrm{CFU}$ ) is re-suspended in fluid A to give $10^{3}$ spores $/ \mathrm{ml}$ of microbial concentration. One milliliter of this sample was added to 
$9 \mathrm{ml}$ of Sabonin Dextrose Broth (SAB), to give an estimated initial load of $\sim 100 \mathrm{CFU} / \mathrm{ml}$ which was later confirmed by the plate counts on Sabonin Dextrose Agar (SDA). This is directly used for impedance measurements without creating a log culture growth as for microorganism in cryotubes. Periodically, (every 1 to $6 \mathrm{hrs}$, depending on expected doubling time of the microorganisms) aliquots from these different media are drawn and assayed electrically using our impedance measurements. Parallel aliquots are plated on appropriate nutrient agar, and colony counts obtained after incubation for suitable length of time.

\section{$\underline{\text { 8.6.3 }} \underline{\text { MPN experiments }}$}

As in the manner described in section 3.2, log-cultures of E. coli (K12) in TSB, with bacterial concentrations $\sim 10^{9} \mathrm{CFU} / \mathrm{ml}$, were obtained. One milliliter of this sample was taken in an Eppendorf tube, centrifuged for $8 \mathrm{mins}$ at $6000 \mathrm{rpm}$ and the pellet (bacteria) is re-suspended in $1 \mathrm{ml}$ of sterile fresh PBS. This is serially diluted in $1 \mathrm{ml}$ of PBS to obtain $1 \mathrm{ml}$ of buffer with $\sim 10^{3} \mathrm{CFU} / \mathrm{ml}$ of $E$. coli. This serves as our "original sample", analogous to the "real world" scenario of a sample of water collected from a source of interest (typically 10L) and concentrated 1000-fold (to 10ml) (Horan 2003).

This $1 \mathrm{ml}$ of "original sample" was then added to $9 \mathrm{ml}$ of sterile TSB (in a sterile $15 \mathrm{ml}$ centrifuge tube) to obtain $10^{2} \mathrm{CFU} / \mathrm{ml}$ of $E$. coli and then serially diluted to obtain $10 \mathrm{CFU} / \mathrm{ml}, 1 \mathrm{CFU} / \mathrm{ml} 0.1 \mathrm{CFU} / \mathrm{ml}$, and $<0.1 \mathrm{CFU} / \mathrm{ml}$. This serial dilution step is repeated to make 3 sets of these same dilutions. This is then incubated at $37^{\circ} \mathrm{C}$ in an incubator shaker (Mini 4450 Shaker, Thermo Scientific ${ }^{\mathrm{TM}}$ ) and every 2 hours, $\sim 250 \mu 1$ of sample is 
taken, injected in the microfluidic cassette and the sample is assayed electrically using our method. The measurements are run for a total of 12 hours.

The traditional MPN protocol (Cochran 1950) is also implemented on the same samples. The samples, from which aliquots are drawn for electrical assay by our method, are incubated for an additional 12 hours (after we have ceased taking our electrical readings) for observation of visible bacterial growth (change of turbidity), as is typically done in MPN experiments. The results obtained by the two methods are then compared.

\section{$\underline{\text { 8.6.4 }} \underline{\text { Multi-frequency impedance measurement and data analysis }}$}

Our method involves drawing a $250 \mu \mathrm{l}$ aliquot at regular time intervals from our suspension of interest, and introducing the aliquot into a microfluidic cassette with electrodes at specified locations. The electrical impedance $(Z)$ between the electrodes is measured at multiple frequencies $(\omega)$, and the $Z$ vs. $\omega$ data is analyzed offline to determine the bulk capacitance $\left(\mathrm{C}_{\mathrm{b}}\right)$ of the suspension.

The impedance $(\mathrm{Z})$ measurements are made using an Agilent 4294A Impedance Analyzer (Agilent technologies, CA, USA) at multiple frequencies ( $\omega)$ from $1 \mathrm{kHz}$ to 100 MHz. To obtain the impedance at a given frequency, the Impedance Analyzer generates a $500 \mathrm{mV}$ (peak to peak) voltage at that frequency, and records the magnitude and phase of the AC current through the sample as a result of the application of the same. It also takes as inputs, the frequency range to be scanned ( $1 \mathrm{kHz}$ to $100 \mathrm{MHz}$ in our case) and the number of points desired (500, the maximum possible, in our case) and selects logarithmically equi-spaced frequencies. It records the measured values of $\mathrm{R}$ and $\mathrm{X}$ 
corresponding to each frequency $(\omega)$ in an ASCII file. The data in this file are analyzed off-line using software ZView ${ }^{\mathrm{TM}}$.

Data obtained from the impedance analyzer is fit to the equivalent circuit of software $\mathrm{ZView}^{\mathrm{TM}}$. The software is written for use in Electrical/Electrochemical Impedance Spectroscopy. It accepts as input measured values of Resistance (R) and Reactance $(\mathrm{X})$ at multiple frequencies (or, equivalently, the magnitude of the Impedance $(\mathrm{Z})$ and the phase angle $(\Theta)$ ), allows the user to propose an equivalent circuit for the material being investigated, and provides an estimate of the values of the individual elements in the equivalent circuit $\left(\mathrm{L}_{\mathrm{e}}, \mathrm{R}_{\mathrm{e}}, \mathrm{C}_{\mathrm{e}}, \mathrm{R}_{\mathrm{b}}, \mathrm{CPE}-\mathrm{T}\right.$, and CPE-P in our case), along with an "error" of the estimate. By providing estimates of each individual parameter, the software allows the user to distinguish changes to the overall impedance occurring due to an increase in the bulk capacitance, from other factors, such as changes to the bulk resistance (for instance, temperature swings can change to the electrical conductivity of the solution, which in turn changes the bulk resistance).

The number of viable microorganisms in a suspension affects the value of the Bulk Capacitance $\left(\mathrm{C}_{\mathrm{b}}\right)$. If the number of microorganisms increase (due to proliferation) then the value of $\mathrm{C}_{\mathrm{b}}$ increases. Conversely, death of some microorganisms will result in a decrease in the value of $\mathrm{C}_{\mathrm{b}}$. For microbial cultures (or any other study where the objective is merely to ascertain whether or not there are any viable microorganisms in a sample of interest), a "significant" increase in the value of $\mathrm{C}_{\mathrm{b}}$ from its value at $\mathrm{t}=0$ implies that there is an increase in the numbers of those physical elements that contribute to the bulk capacitance. Hence a significant increase in $\mathrm{C}_{\mathrm{b}}$, if observed, serves to confirm the 
presence of microorganisms in the original sample and when such an increase is observed, the sample is deemed positive by our method.

The use of this criterion is illustrated in Figure 8.2. As seen, initially (at $t=0$ ), the magnitude of the CPE (blue diamond) is $1.96 \pm 0.024$ Pico-Farads. The range of values is represented by the error bars. The value of Bulk Capacitance after 6 hours of incubation (obtained based on impedance measurements taken at that time) is $1.99 \pm 0.024$ PicoFarads. As seen, the range overlaps with that of the 0-hour value (shaded region). Thus, while we know in retrospect (from the colony counts of aliquots drawn at the time the impedance measurements are taken) that the number of bacteria increased (from $\sim 10$ to $\sim 20 \mathrm{CFU} / \mathrm{ml}$ of suspension), the increase did not cause a "significant" change in the value of the Bulk Capacitance. Similarly, for the 12, and 18 hour readings, the bulk capacitance value did not change significantly compared to the 0 hour. However, the magnitude of the $\mathrm{CPE}$ at the 24 hour mark $(2.07 \pm 0.024$ Pico-Farads $)$ is "significantly" different from the 0 -hour value $(2.07-0.024=2.046>1.984=1.96+0.024)$. Thus 24 hours is our Time to Detection (TTD) for this sample (indicated by the solid blue arrow). The threshold concentration can be estimated from the plate count obtained from the sample plated at TTP. In the case shown in Figure 8.2, it is (as indicated by the red dotted arrow) 1000 $\mathrm{CFU} / \mathrm{ml}$. 


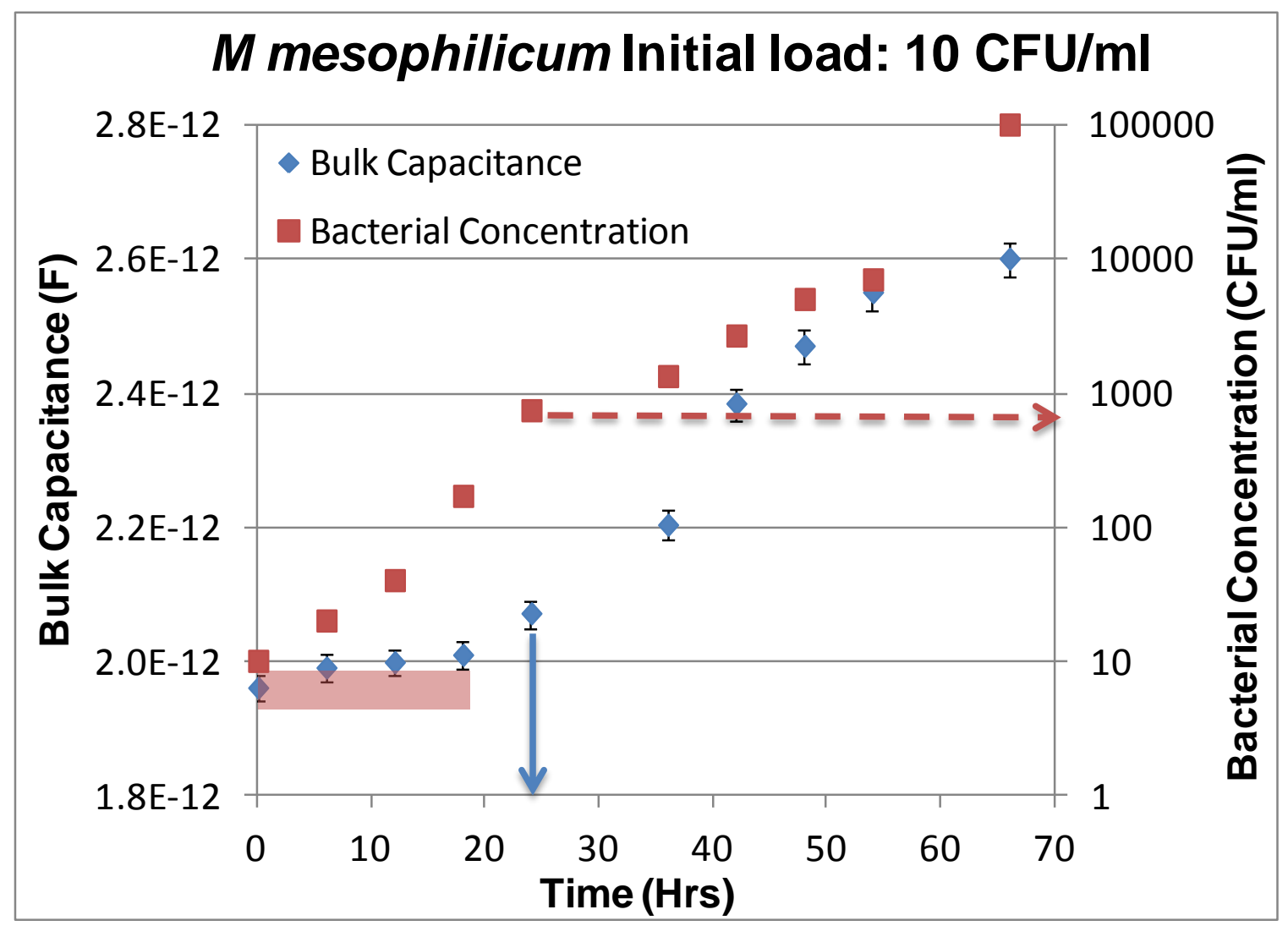

Figure 8.2: Plot of M mesophilicum bulk capacitance values over time

Plot showing how the magnitude of the CPE of the bulk solution (blue diamonds) and the concentration of microorganisms (red squares) evolve over time during culture in a sample with an initial load of $\sim 10$ CFU/ml of M mesophilicum. While microbial concentrations increase monotonically, magnitudes of CPE measured at 6, 12, and 18 hrs do not differ significantly from the 0-hr value (error bars overlap). A significant increase recorded only at 24 hrs, making this the Time to Detection (TTD) (solid blue arrow). The microbial concentration in the culture broth at TTD, the "threshold concentration", for this sample is $\sim 1000 \mathrm{CFU} / \mathrm{ml}$, as shown by the red dashed line.

For determining MPN, the same principle is applied individually to all (diluted) samples to determine if there are any viable bacteria present, or not. Since our threshold concentrations are lower than that of current methods, we can detect the presence of bacteria much earlier, and also deem a sample to be negative in a correspondingly shorter time ( $8 \mathrm{hrs,}$ for the experiments conducted). The number of positives and negatives at 
each dilution are recorded, and using statistical tables available in literature (Salama et al. 1978), the MPNs estimated.

\subsection{Results and Discussion}

\section{$\underline{8.7 .1} \underline{\text { Rapid detection of viable microorganisms }}$}

In Figure 8.3, we graphically present data from experiments conducted for all the microorganisms mentioned, with two different initial loads shown for each. Each graph shows the bulk capacitance values (blue diamonds) obtained by analyzing the electrical scans conducted at different points in time using the method described in Section 8.3. The error-bars represent the standard error of the estimate, and are also provided by the impedance-analysis software $\left(\mathrm{ZView}^{\mathrm{TM}}\right)$ used. Actual concentrations of the microorganisms in the culture broth at these times (found using plate culture, as described in section 8.2) are represented by red squares. The times to detection for each sample (obtained using the criteria described in Section 8.4) are highlighted using a blue solid arrow, and the threshold concentration (also obtained as described in Section 8.4) is highlighted using the dashed red arrow. Table 8.2 gives the TTDs and the threshold concentrations for the different initial loads of the microorganisms tested. For the samples tested, the threshold concentration is of the order of $10^{3} \mathrm{CFU} / \mathrm{ml}$, irrespective of the identity of the organism. This threshold concentration is significantly lower than the

threshold concentrations of current detection systems $\left(10^{7}-10^{8} \mathrm{CFU} / \mathrm{ml}\right)($ Smith et al. 2008). 


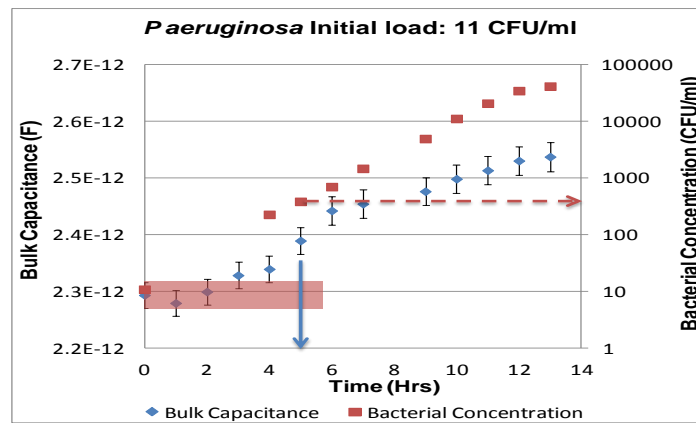

M mesophilicum Initial load: 1 CFU/mI

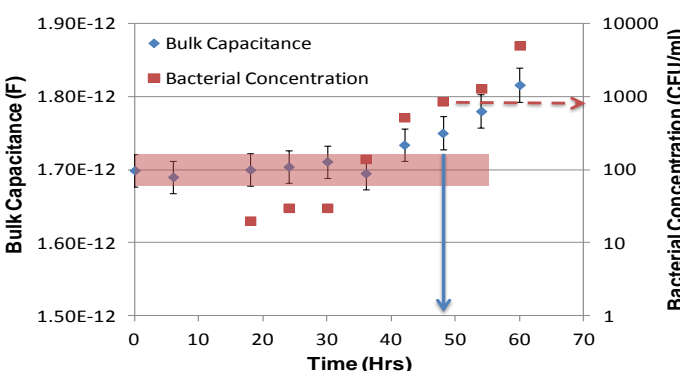

$P$ acnes Initial load: 50 CFU/ml

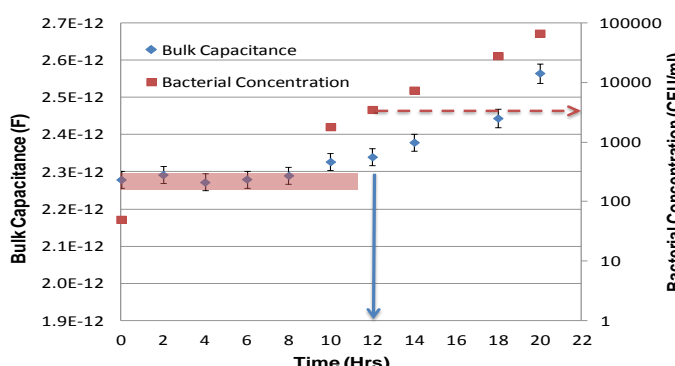

A brasiliensis Initial load: 5 CFU/ml

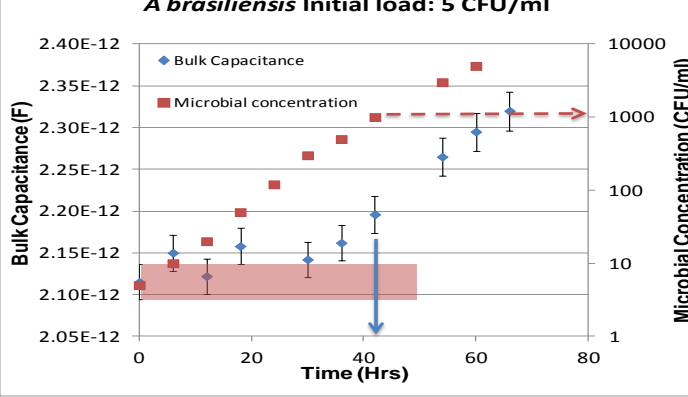

D anomala Initial load: 4 CFU/ml

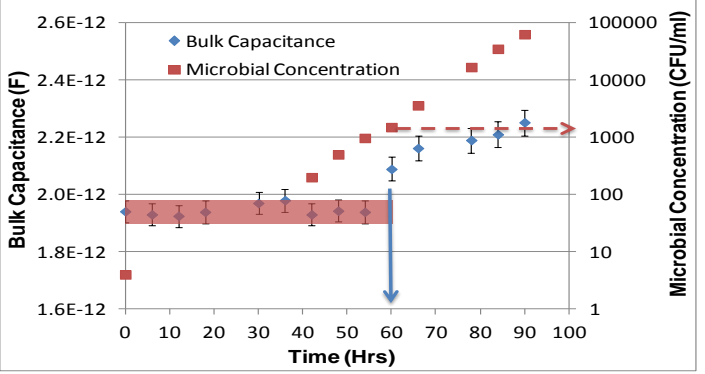

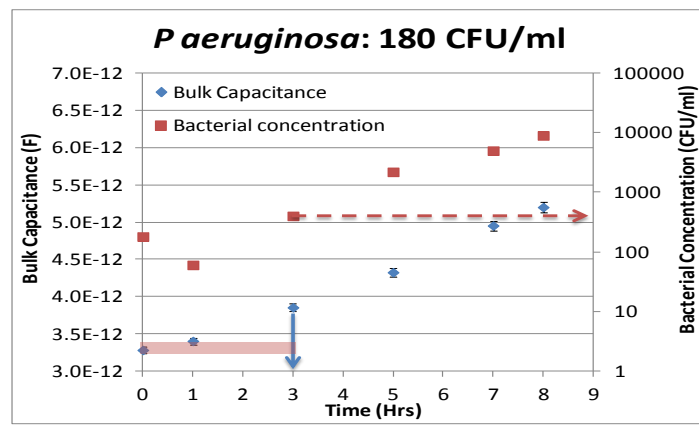

M mesophilicum Initial load: 10 CFU/ml

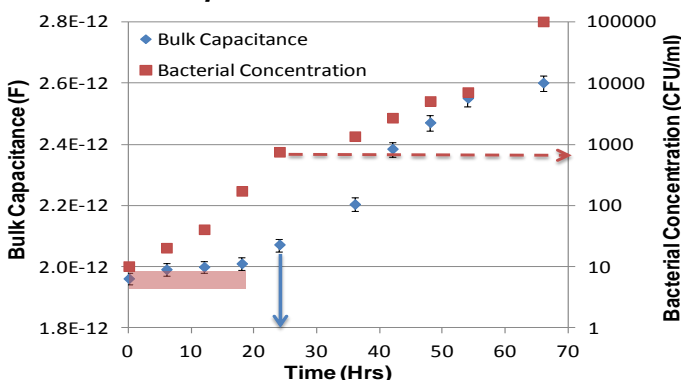

$P$ acnes Initial load: $100 \mathrm{CFU} / \mathrm{ml}$

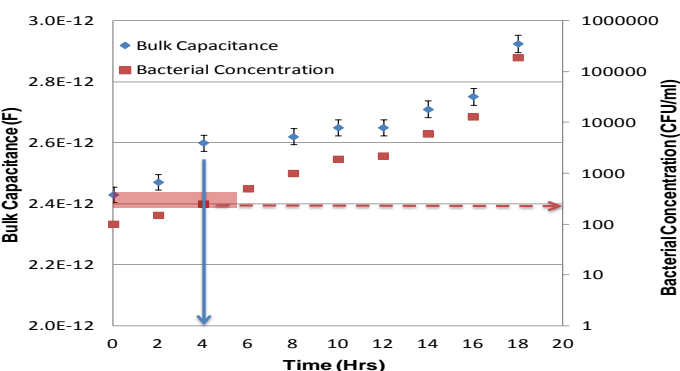

A brasiliensis Initial load: $10 \mathrm{CFU} / \mathrm{ml}$

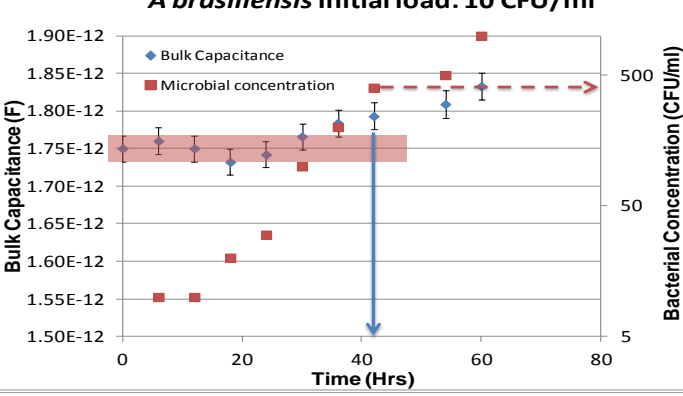

D anomala Initial Load: $200 \mathrm{CFU} / \mathrm{ml}$

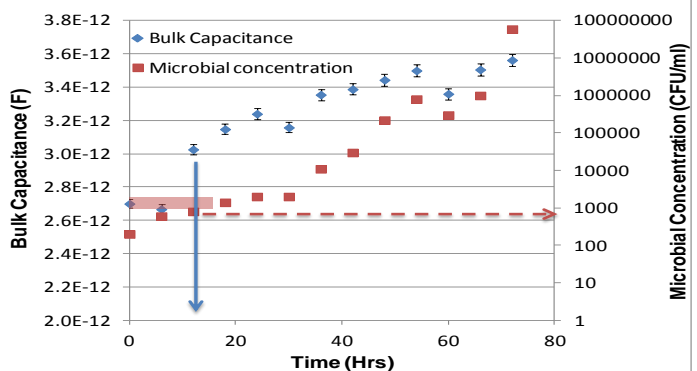

Figure 8.3: Plot of results obtained for all different microorganisms 
Graphical representation of the results obtained using our method of detection for all the microorganisms, each with 2 different initial loads. Each graph represents the bulk capacitance values (Blue diamonds) and corresponding microbial concentrations (Red squares) plotted over time. The blue solid arrow represents the TTD value for each microorganism at the given initial load, while the red dashed arrow represents the threshold concentration at the time of detection.

\subsubsection{Dependence of TTD on initial load $\left(\mathrm{n}_{0}\right)$ and doubling time $\left(\mathrm{t}_{\underline{D}}\right)$ of the} $\underline{\text { micro-organism }}$

In Table 8.2, it can be observed that Times to Detection (TTDs) are longer with for lower initial loads for any given microorganism. Similarly, when comparing samples with similar initial loads $\left(\mathrm{n}_{0}\right)$, microorganisms with a shorter doubling time $\left(\mathrm{t}_{\mathrm{D}}\right)$ such as Pseudomonas aeruginosa (for which $\mathrm{t}_{\mathrm{D}}=1 \mathrm{hr}($ Pearson et al. 1995)) have a shorter TTD compared to microorganisms with a longer doubling time, such as Methylobacterium mesophilicum (for which $\mathrm{t}_{\mathrm{D}}=8 \mathrm{hrs}$ (Sy et al. 2001)). This is because the microorganisms can be detected (caught in the act of increasing their numbers through reproduction) only when they have reached a certain threshold concentration. When we start with a low initial load, it takes longer to reach the threshold concentration and hence the samples have a longer TTD. Similarly, for similar initial loads, microorganisms with longer doubling-times need a longer amount of time to reach the threshold concentration. Hence, there is a strong correlation between the doubling-times, initial loads of the microorganism and the TTDs. 


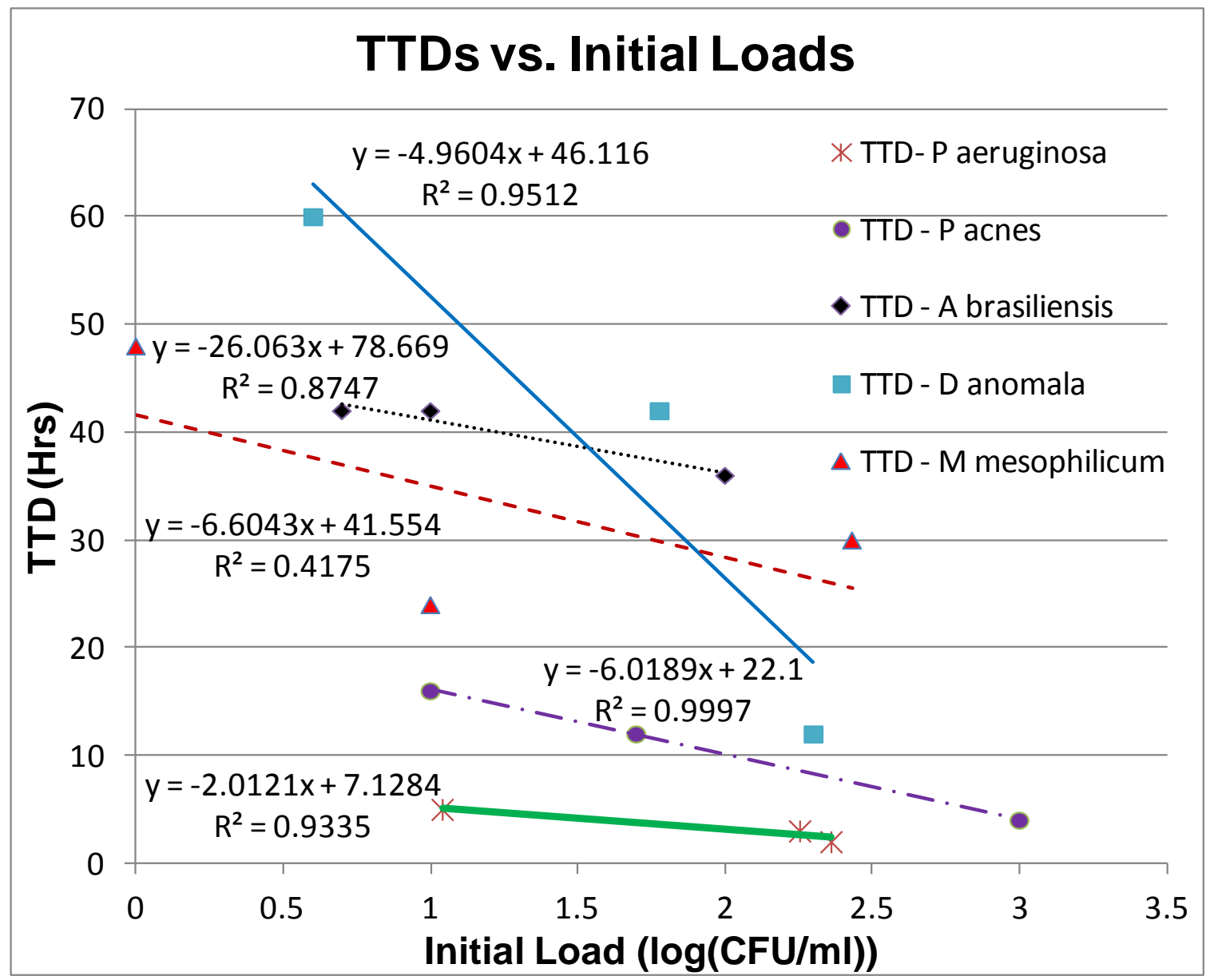

Figure 8.4: TTDs vs. Initial loads for all microorganisms used

Represents the plot of TTDs for all the sample microorganisms at different initial loads. The solid straight lines represent the regression lines of fit for each microorganism along with the equation of line

If the surmise above is true, then mathematically, the Time to Detection (TTD) should be related to the initial load $\left(\mathrm{n}_{0}\right)$ and doubling time $\left(\mathrm{t}_{\mathrm{D}}\right)$ of the microorganism by the equation:

$\mathrm{TTD}=1.443 \mathrm{t}_{\mathrm{D}} \ln \left(\mathrm{n}_{\mathrm{T}} / \mathrm{n}_{0}\right)$ 
In our experiments, the initial load in the sample and the threshold concentration can be estimated from the plate counts obtained at time $\mathrm{t}=0$, and time $\mathrm{t}=\mathrm{TTD}$ (as determined by analyzing the electrical data), respectively. By plotting the experimentally obtained values of TTD and $\mathrm{n}_{\mathrm{T}}$ for different samples (different $\mathrm{n}_{0}$ values) of the same organism, we should be able to estimate its doubling time ( $\left.t_{D}\right)$. If the estimated $t_{D}$ is found to be "reasonable", this would indicate that our system behaves in the manner that is consistent with our understanding.

The above equation may be re-written as

$$
\mathrm{TTD}=3.32 \mathrm{t}_{\mathrm{D}} \log _{10}\left(\mathrm{n}_{\mathrm{T}}\right)-3.32 \mathrm{t}_{\mathrm{D}} \log _{10}\left(\mathrm{n}_{0}\right)
$$

Thus, if we plot TTD (y) of each sample against the $\log _{10}$ of initial loads (x), a regression analysis provides us with not just the values of the slope and intercept, but also the $90 \%$ Confidence Intervals (CIs) of the same. Using the intercept values obtained from the regression line fit, and assuming an average threshold concentration of $1000 \mathrm{CFU} / \mathrm{ml}$ for all organisms, the doubling time $\left(\mathrm{t}_{\mathrm{D}}\right)$ for each microorganism along with $90 \% \mathrm{CI}$ is calculated using Equation 8.4:

$$
t_{D}=\text { Intercept } /\left(3.32 * \log _{10}\left(\mathrm{n}_{\mathrm{T}}\right)\right)
$$

We chose to use an "average" value of the threshold (rather than individual estimates for different experiments) because estimates obtained by standard plate counts itself is best regarded as an order-of-magnitude estimate (Bhupathiraju et al. 1999). The calculated values of doubling time $\left(t_{D}\right)$ in hours along with the $90 \%$ CI values (upper and lower limit values) are given in Table 8.2. The expected doubling time for each of these 
microorganisms are also given in the same table for comparison. It can be seen that the calculated doubling times (their 90\% CIs) lie within the expected range for the doubling times for all microorganisms. Minor discrepancy in the values is expected as there is likely to have been an initial lag phase during microbial growth and also that the exact threshold concentration is likely to be slightly different for each microorganism. 


\begin{tabular}{|c|c|c|c|c|c|}
\hline Microorganism & $\begin{array}{c}\text { Initial Load } \\
\text { (CFU/ml) }\end{array}$ & $\begin{array}{c}\text { Time to } \\
\text { Detection } \\
\text { (Hrs) }\end{array}$ & $\begin{array}{c}\text { Threshold } \\
\text { Concentration } \\
\text { (CFU/ml) }\end{array}$ & $\begin{array}{c}\text { Calculated Doubling } \\
\text { time (Hrs) } \\
\text { [90\% Confidence } \\
\text { Interval] }\end{array}$ & \begin{tabular}{|l|} 
Expected \\
doubling \\
time (Hrs)
\end{tabular} \\
\hline$P$ aeruginosa & $\begin{array}{c}11 \\
180 \\
230\end{array}$ & $\begin{array}{l}5 \\
5 \\
2\end{array}$ & $\begin{array}{c}390 \\
2100 \\
870\end{array}$ & $\begin{array}{c}0.72 \\
(0.04-1.39)\end{array}$ & \begin{tabular}{|c|}
$0.75-1$ \\
(Pearson et \\
al. 1995)
\end{tabular} \\
\hline M mesophilicum & $\begin{array}{c}1 \\
10 \\
270\end{array}$ & $\begin{array}{l}48 \\
24 \\
30\end{array}$ & $\begin{array}{r}1000 \\
750 \\
4440\end{array}$ & $\begin{array}{c}4.2 \\
(-3.3-11.6)\end{array}$ & $\begin{array}{l}8-12 \text { (Sy } \\
\text { et al. 2001) }\end{array}$ \\
\hline Pacnes & $\begin{array}{c}10 \\
50 \\
1000\end{array}$ & $\begin{array}{l}16 \\
12 \\
4\end{array}$ & $\begin{array}{r}2600 \\
3500 \\
1900\end{array}$ & $\begin{array}{c}2.22 \\
(2.07-2.35)\end{array}$ & $\begin{array}{l}1-2 \text { (Hall } \\
\text { et al. 1994) }\end{array}$ \\
\hline A brasiliensis & $\begin{array}{c}5 \\
10 \\
100\end{array}$ & $\begin{array}{l}42 \\
42 \\
36\end{array}$ & $\begin{array}{r}1000 \\
400 \\
1500\end{array}$ & $\begin{array}{c}4.63 \\
(3.66-5.59)\end{array}$ & 6 \\
\hline D anomala & $\begin{array}{c}4 \\
60 \\
200\end{array}$ & $\begin{array}{l}60 \\
42 \\
12\end{array}$ & $\begin{array}{c}1500 \\
10000 \\
800\end{array}$ & $\begin{array}{c}7.88 \\
(-2.8-18.57)\end{array}$ & $\begin{array}{c}6 \text { (Abbott et } \\
\text { al. 2005) }\end{array}$ \\
\hline
\end{tabular}

Table 8.2: TTDs for different initial loads of microorganisms and their calculated doubling times

\subsubsection{Determining the Most Probable Number (MPN) of viable bacteria}

Each of the graphs shown in Figure 8.5, show how the Bulk Capacitance values of each of the 3 "tubes" at a given dilution of the "original sample" change with time. If a significant change in Bulk Capacitance is not observed within the duration for which 
measurements are taken (12 hours), then the particular tube is deemed negative. On the other hand, tubes are deemed positive as soon as a significant change in Bulk Capacitance is recorded. 

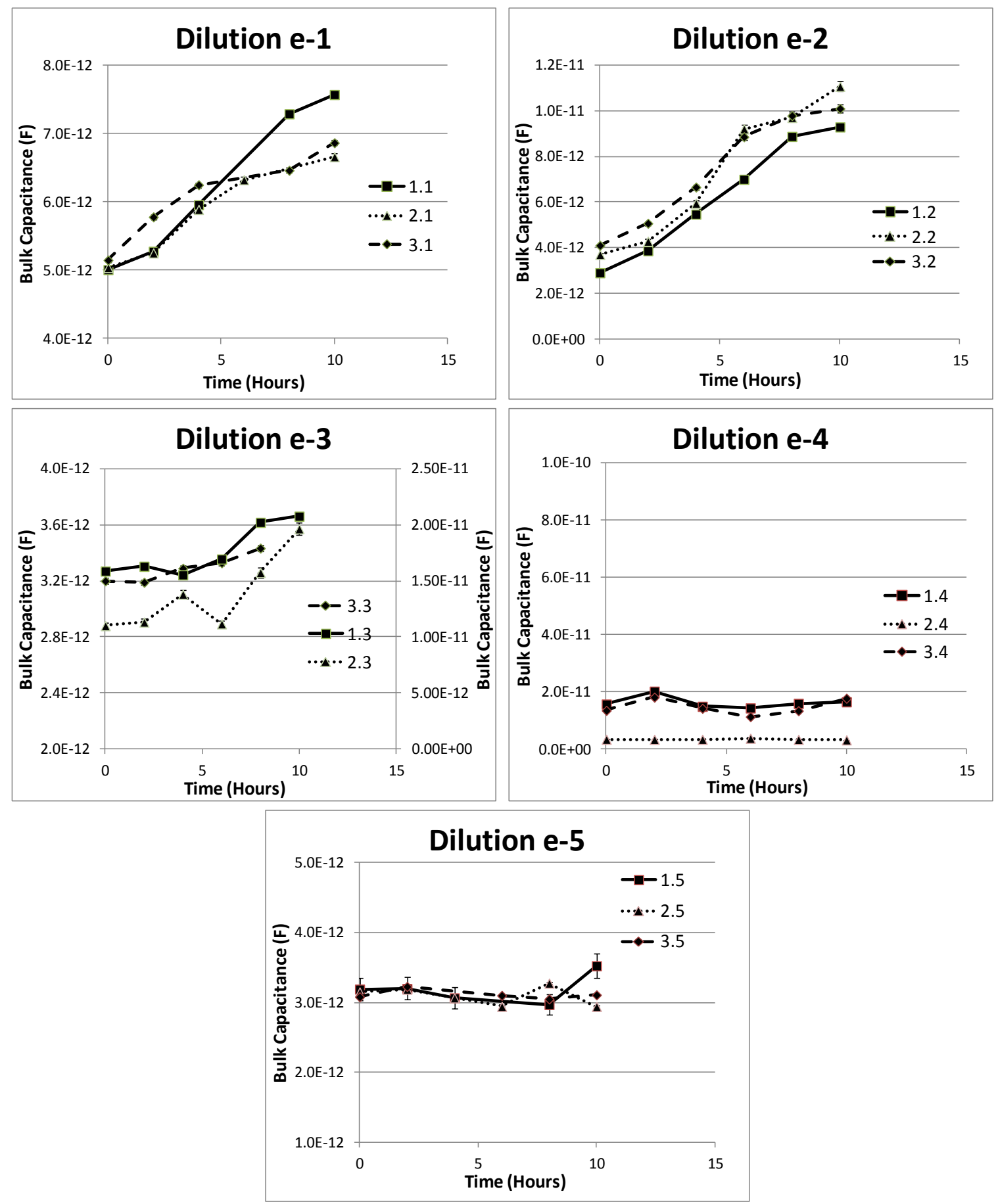

Figure 8.5: MPN results using our method

Plots showing changes in the value of the measured Bulk Capacitance over time for samples containing different dilutions (e-1,e-2,e-3,e-4 and e-5) of the original sample (containing $\sim 1000 \mathrm{CFU} / \mathrm{ml}$ ) with the arrows indicating the TTD for the given dilution. 
From the plots shown in Figure 8.5 it can be seen that for all the tubes in the $1^{\text {st }}$ three dilution series (which are expected to contain $\sim 100, \sim 10$ and $\sim 1 \mathrm{CFU} / \mathrm{ml}$ ), we can observe an increase in the value of the bulk capacitance (blue diamonds) over time indicating the presence of bacteria in these tubes. This was also confirmed by presence of visible growth in the tubes after 24 hours of incubation. While for the $4^{\text {th }}$ and the $5^{\text {th }}$ dilution sets, with expected bacterial concentrations of $\sim 0.1 \mathrm{CFU} / \mathrm{ml}(\sim 1 \mathrm{CFU} / 10 \mathrm{ml})$, and $\sim 0.01 \mathrm{CFU} / \mathrm{ml}(<1 \mathrm{CFU} / 100 \mathrm{ml})$, there was no observed increase in the bulk capacitance values and also the TSB did not turn cloudy after $24 \mathrm{hrs}$ of incubation (indicating the lack of bacteria).

The bacterial culture results (whether positive or negative) for all tubes are summarized in Table 8.3. For any given dilution, the number of tubes turning positive using the traditional evaluation method (check for turbidity in the originally-clear medium after 24 hours of culture) is identified. This is compared (as shown in the table) to the result (positive or negative) obtained using our method. As seen, we obtained the "correct" result for all cases, and in all them, the positive samples were identified using our method in 2-6 hrs. (The solid blue lines in the graphs indicate the TTP for that particular tube). 


\begin{tabular}{|c|c|c|c|c|}
\hline \multirow[b]{3}{*}{ Dilution Factor $\left(\log _{10}\right)$} & \multirow{3}{*}{$\begin{array}{c}\text { Tube } \\
\text { Number }\end{array}$} & \multicolumn{3}{|c|}{ RESULTS } \\
\hline & & \multirow{2}{*}{$\begin{array}{c}\text { Traditional MPN } \\
\text { (turbidity after } 24 \text { hrs } \\
\text { of incubation) }\end{array}$} & \multicolumn{2}{|c|}{ Our method } \\
\hline & & & Result & $\begin{array}{l}\text { Time to } \\
\text { Positivity } \\
\text { (TTP) }\end{array}$ \\
\hline \multirow{3}{*}{-1} & 1 & Positive & Positive & 2 hours \\
\hline & 2 & Positive & Positive & 2 Hours \\
\hline & 3 & Positive & Positive & 2 Hours \\
\hline \multirow{3}{*}{-2} & 1 & Positive & Positive & 2 Hours \\
\hline & 2 & Positive & Positive & 2 Hours \\
\hline & 3 & Positive & Positive & 2 Hours \\
\hline \multirow{3}{*}{-3} & 1 & Positive & Positive & 6 Hours \\
\hline & 2 & Positive & Positive & 4 Hours \\
\hline & 3 & Positive & Positive & 4 Hours \\
\hline \multirow{3}{*}{-4} & 1 & Negative & Negative & --- \\
\hline & 2 & Negative & Negative & --- \\
\hline & 3 & Negative & Negative & --- \\
\hline \multirow{3}{*}{-5} & 1 & Negative & Negative & --- \\
\hline & 2 & Negative & Negative & --- \\
\hline & 3 & Negative & Negative & --- \\
\hline
\end{tabular}

Table 8.3: Comparison of results obtained using our method and the traditional method for $M P N$

In order to estimate the concentration of bacteria in the original sample, we identified 3 sets of dilution tubes which show the dilution of organisms "to extinction" - 
i.e., 3 successive sets of tubes where the indication for bacterial presence goes from positive to negative. For our experiment, it was [3, 0, and 0]. Now, using the 3 tube MPN table, and the obtained [3,0, and 0] result, we can determine the MPN of bacteria in the samples to be 0.23 in the center tube (with a dilution factor of $\mathrm{e}^{-4}$ with respect to the original sample). Hence, the Most Probable Number (MPN) of organisms in the original undiluted sample to be is $2.3 \times 10^{3} \mathrm{CFU} / \mathrm{ml}$.

These results suggest that our method could be used to evaluate MPN significantly faster than traditional methods. In fact, the results suggest that a "cutoff time" of as low as $6 \mathrm{hrs}$ can be used for coliforms (as opposed to 1-2 days, currently). This could have significant impact in a number of situations (such as those related to food quality and recreational water, as explained earlier).

\subsection{Conclusions}

In our previous work we had reported our ability to detect viable (proliferating) bacteria in food substrates (Puttaswamy and Sengupta 2010b) and blood cultures (Puttaswamy et al. 2011) in a significantly (4-10 fold) shorter amount of time, when compared to existing culture-based methods (automated culture systems). In this work, we have described in detail the protocols we used to acquire and analyze the data, and have explained the mathematical criteria that we used to identify samples in which viable (proliferating) microorganisms are present. More importantly, we have shown that the method is applicable to a wide variety of microorganisms (aerobic and anaerobic bacteria, fastidious bacteria, yeasts and molds), and all these microorganisms have similar 
detection thresholds, thereby making our method one suitable to a variety of applications. That the doubling times, calculated based on our hypothesized mathematical model for the behavior of the system and experimentally estimated threshold concentrations, are in line with expectations confirms that our understanding of how our system behaves is valid. In addition, we have presented "proof of principle" results showing how our basic method can also be adapted to determine the MPN of samples rapidly (again, 4-10 times more quickly than currently done).

As currently implemented, our system has one major drawback: viz. the need for periodic sampling. Manual sampling is not only tedious; it may also make the process economically non-viable. Moreover, there is always the chance of contamination being introduced when aliquots are drawn. Therefore, we are currently in the process of developing an automated aseptic sampling system that will not require manual intervention for the duration of the culture (hours to days). Once this system is developed, we plan on performing more experiments, especially with "unknown" or "field" samples, to establish the effectiveness of the method. 


\section{CHAPTER 9}

\section{CONCLUSION AND FUTURE WORK}

The research project presented here represents a novel approach to rapidly detect a wide range of proliferating microorganisms, quantify them and characterize the antibiotic resistance properties. The method that is used for the detection is based on detecting the change in bulk capacitance over time, of the suspension containing microorganisms, when supplied with a AC electric field. For this purpose, a microfluidic cassette designed specifically to have a long narrow channel was used to amplify the effect of bulk capacitance of the suspension. Since the bacteria in the presence of the AC electric field can act as capacitors, an increase in their number will lead to a corresponding increase in the capacitance of the suspension, while a decrease in its number will lead to a decrease in the capacitance values. This is able to closely and directly track the presence of viable microorganisms in a given sample.

This approach is significantly different in comparison to most of the automated systems currently being used, as well as the ones under development. These methods mainly rely on monitoring the changes in the media such as changes in $\mathrm{pH}$, conductivity, $\mathrm{CO}_{2}$ release, etc. observed to change in microbial metabolism, which takes a significantly longer time. This is because when one starts with significantly low concentrations of bacteria, the amount of metabolites consumed/released is very small and one has to wait for the bacteria to reach a relatively high number before a significant change in the parameter being detected is observed. 
On the other hand, since our method is able to detect the change in capacitance as the bacteria proliferate, it takes a significantly shorter time for detection. We were also able to demonstrate the feasibility of this system to detect presence of viable bacteria food samples and in blood cultures at initial loads of $1-1000 \mathrm{CFU} / \mathrm{ml}$ in $4-10 \mathrm{X}$ faster than the current methods such as BACTEC. Since a decrease in the bacterial number (due to cell death) will lead to a decrease in bulk capacitance, we were able to take advantage of it in antibiotic susceptibility testing of various bacteria. Our method was used to determine the MIC of certain candidate antibiotics against 3 different bacterial strains in 4 hours and simultaneously was able to infer the type of effect (bacteriostatic or bactericidal) the antibiotic has on the specific bacteria. We were able to successfully demonstrate that this method can be used for detection of various sample microorganisms (aerobic, anaerobic, yeast and mold) at different initial loads ranging from $1 \mathrm{CFU} / \mathrm{ml}$ to $1000 \mathrm{CFU} / \mathrm{ml}$ and that it can be used as a faster method for quantifying the presence of viable bacteria in liquid samples such as water, food products, etc.

However, as it stands, the current system under development has a major drawback that it needs periodic sampling (every 1 hour or so depending on the type of microbe used). This manual sampling and readings consumes time, makes the process more tedious, labor intensive and economically non-viable. Apart from these, there is also a high chance of contamination in following the current manual method. Hence, we are currently working towards the development of a single-unit aseptic automated device for sample processing and readings. This work is being conducted in collaboration with Techshot Inc. who have developed an initial working device which can, once the media with bacteria is added to the system, at regular time intervals (currently set to a period of 
20 mins) automatically draw a small volume of the sample into a tube, take electrical readings across this tube and saves the data. The data can then be analyzed to determine the bulk capacitance values over time. This system currently has been tested for its ability to maintain sterility for prolonged time periods (5 days) while running samples and has been successful in doing so. The current electrical system on this device however, needs further refining after which a multi-unit system will be built which is capable of running several samples in parallel. The ability of our method to rapidly detect the presence of low concentrations of a viable microorganism coupled with the design of an automated system with the make and feel of the system similar to the ones that are currently being used, makes this method a significant advancement in the field of microbial detection. 


\section{REFERENCES}

2004. US Pharmacopeial Convention. General Chapter <61>, "Microbial Limit Tests", USP 27, Rockville, MD.

Impedance Spectroscopy: Theory, Experiment, and Applications 2nd edition E. Barsoukov and J.R. Macdonald, Editors, 2005. John Wiley \& Sons, Inc, New Jersey.

2005b. Impedance Spectroscopy: Theory, Experiment, and Applications, 2nd edition ed. John Wiley \& Sons, Inc, New Jersey.

2009. Outbreak of Listeria monocytogenes Infections Associated With Pasteurized Milk From a Local Dairy-Massachusetts-2007. Journal of american medical association 301(8), 820-822.

Abbott, D., Hynes, S., Ingledew, W., 2005. Growth rates of Dekkera/Brettanomyces yeasts hinder their ability to compete with Saccharomyces cerevisiae in batch corn mash fermentations. Applied microbiology and biotechnology 66(6), 641-647.

Ahmad, A.S., Puttaswamy, C., Mudasser, S., Abdelaziz, O., 2011. Clinical Presentation and Outcome in Hospitalized Patients of 2009 Pandemic Influenza A (H1N1) viral infection in Oman. Oman Med J 26(5), 329-336.

Andersen, K., von Meyenburg, K., 1980a. Are growth rates of Escherichia coli in batch cultures limited by respiration? Journal of Bacteriology 144(1), 114.

Andersen, K.B., Von Meyenburg, K., 1980b. Are growth rates of Escherichia coli in batch cultures limited by respiration? Journal of Bacteriology 144(1), 114-123.

Anderson, K.A., Davidson, P.M., 1997. Drinking Water \& Recreational Water Quality: Microbiological Criteria. University of Idaho, College of Agriculture, Cooperative Extension System, Agricultural Experiment Station.

Angus, D.C., et al.,, 2001. Epidemiology of sever sepsis in the United States: Analysis of incidence, outcome, and associated costs of care. Critical Care Medicine 29(7), 1303-1310.

Asami, K., 2002. Characterization of biological cells by dielectric spectroscopy. Journal of NonCrystalline Solids 305(1-3), 268-277.

Baboian, R., 2005. Corrosion Tests And Standards: Application And Interpretation, Second ed. ASTM international.

Bhupathiraju, V.K., Hernandez, M., Krauter, P., Alvarez-Cohen, L., 1999. A new direct microscopy based method for evaluating in-situ bioremediation. Journal of hazardous materials 67(3), 299312.

Bolton, F., 1990. An investigation of indirect conductimetry for detection of some food-borne bacteria. Journal of Applied Microbiology 69(5), 655-661.

Borsheim, K., Bratbak, G., Heldal, M., 1990a. Enumeration and biomass estimation of planktonic bacteria and viruses by transmission electron microscopy. Applied and Environmental Microbiology 56(2), 352.

Borsheim, K.Y., Bratbak, G., Heldal, M., 1990b. Enumeration and biomass estimation of planktonic bacteria and viruses by transmission electron microscopy. Applied and Environmental Microbiology 56(2), 352-356. 
Brian, M., Frosolono, M., Murray, B., Miranda, A., Lopez, E., Gomez, H., Cleary, T., 1992. Polymerase chain reaction for diagnosis of enterohemorrhagic Escherichia coli infection and hemolytic-uremic syndrome. Journal of Clinical Microbiology 30(7), 1801-1806.

Buckland, A., Kessock-Philip, S., Bascomb, S., 1983. Early detection of bacterial growth in blood culture by impedance monitoring with a Bactometer model 32. British Medical Journal 36(7), 823-828.

Buzby, J.C., Roberts, T., 1997. Economic costs and trade impacts of microbial foodborne illness. World health statistics quarterly. Rapport trimestriel de statistiques sanitaires mondiales 50(12), 57.

Byrne, B., Stack, E., Gilmartin, N., O’Kennedy, R., 2009. Antibody-based sensors: Principles, problems and potential for detection of pathogens and associated toxins. Sensors 9(6), 44074445 .

Chen, C.H., Lu, Y., Sin, M.L.Y., Mach, K.E., Zhang, D.D., Gau, V., Liao, J.C., Wong, P.K., 2010. Antimicrobial susceptibility testing using high surface-to-volume ratio microchannels. Analytical chemistry 82(3), 1012-1019.

Chung, C.C., Cheng, I.F., Chen, H.M., Kan, H.C., Yang, W.H., Chang, H.C., 2012. Screening of Antibiotic Susceptibility to $\beta$-Lactam-Induced Elongation of Gram-Negative Bacteria Based on Dielectrophoresis. Analytical Chemistry-Columbus 84(7), 3347.

Cochran, W.G., 1950. Estimation of bacterial densities by means of the" most probable number". Biometrics, 105-116.

Cooper, S., Helmstetter, C., 1968. Chromosome replication and the division cycle of Escherichia coli B/r* 1. Journal of Molecular Biology 31(3), 519-540.

Coyette, J., Shockman, G.D., 1973. Some properties of the autolytic N-acetylmuramidase of Lactobacillus acidophilus. Journal of Bacteriology 114(1), 34-41.

Craig, W.A., 2001. Does the dose matter? Clinical infectious diseases 33(Supplement 3), S233S237.

Cussler, E.L., 1997. Heat Transfer. Diffusion: Mass Transfer in Fluid Systems, Second ed. Cambridge University Press.

Dalgaard, P., Mejlholm, O., Huss, H.H., 1996. Conductance method for quantitative determination of Photobacterium phosphoreum in fish products. Journal of Applied Microbiology 81(1), 57-64.

Dalton, C.B., Austin, C.C., Sobel, J., Hayes, P.S., Bibb, W.F., Graves, L.M., Swaminathan, B., Proctor, M.E., Griffin, P.M., 1997. An outbreak of gastroenteritis and fever due to Listeria monocytogenes in milk. pp. 100-106.

Darrell, J., Waterworth, P.M., 1967. Dosage of gentamicin for pseudomonas infections. British medical journal 2(5551), 535-537.

De Oliveira, A., Watts, J., Salmon, S., Aarestrup, F.M., 2000. Antimicrobial Susceptibility of< i> Staphylococcus aureus $</ \mathrm{i}>$ Isolated from Bovine Mastitis in Europe and the United States. Journal of dairy science 83(4), 855-862.

Dombrovskiy, V., Martin, A., Sunderram, J., Paz, H., 2007. Rapid increase in hospitalization and mortality rates for severe sepsis in the United States: A trend analysis from 1993 to 2003*. Critical care medicine 35(5), 1244.

Dorfman, M.H., Stoner, N., Rosselot, K.S., Council, N.R.D., 2009. Testing the waters: a guide to water quality at vacation beaches. Natural Resources Defense Council.

Doyle, M., 2007. Microbial food spoilage-Losses and control strategies. FRI briefings. University of Wisconsin, Madison, WI. 
Drake, M.A., Hayes, W., White, C.H., 2002. Sensory Aroma Characteristics of Milk Spoilage by Pseudomonas Species. Journal of food science 67(2), 861.

Dromigny, E., Bourrion, F., Rugraf, Y., Bolton, F.J., Leden, N., 1997. New media for detection and counting of clostridia in foods. Letters in applied microbiology 24(1), 19-22.

Eden, G., Eden, R., 1984. Enumeration of microorganisms by their dynamic AC conductance patterns. Biomedical Engineering, IEEE Transactions on(2), 193-198.

Fass, R., Barnishan, J., 1979. Minimal inhibitory concentrations of 34 antimicrobial agents for control strains Escherichia coli ATCC 25922 and Pseudomonas aeruginosa ATCC 27853. Antimicrobial agents and chemotherapy 16(5), 622-624.

Felice, C.J., Madrid, R.E., Olivera, J.M., Rotger, V.I., Valentinuzzi, M.E., 1999. Impedance microbiology: quantification of bacterial content in milk by means of capacitance growth curves. Journal of microbiological methods 35(1), 37-42.

Felice, C.J., Valentinuzzi, M.E., 1999. Medium and interface components in impedance microbiology. IEEE transactions on biomedical engineering 46(12), 1483-1487.

Finberg, R.W., Moellering, R.C., Tally, F.P., Craig, W.A., Pankey, G.A., Dellinger, E.P., West, M.A., Joshi, M., Linden, P.K., Rolston, K.V., 2004. The importance of bactericidal drugs: future directions in infectious disease. Clinical infectious diseases 39(9), 1314-1320.

Firstenberg-Eden, R., Zindulis, J., 1984. Electrochemical changes in media due to microbial. Journal of microbiological methods 2(2), 103-115.

Friedman, G., Silva, E., Vincent, J., 1998. Has the mortality of septic shock changed with time? Critical care medicine 26(12), 2078.

Gasol, P.A.d.G.a.J.M., 2008. Physiological structure and single-cell activity in marine bacterioplankton. In: Mitchell, R. (Ed.), Microbial Ecology of oceans. John Wiley \& Sons.

Goh, S., Newman, C., Knowles, M., Bolton, F.J., Hollyoak, V., Richards, S., Daley, P., Counter, D., Smith, H.R., Keppie, N., 2003. E. coli 0157 phage type 21/28 outbreak in North Cumbria associated with pasteurized milk. Epidemiology and infection 129(03), 451-457.

Gomez-Sjoberg, R., Morisette, D.T., Bashir, R., 2005. Impedance microbiology-on-a-chip: microfluidic bioprocessor for rapid detection of bacterial metabolism. Microelectromechanical Systems, Journal of 14(4), 829-838.

Gomez, R., Bashir, R., Bhunia, A.K., 2002. Microscale electronic detection of bacterial metabolism. Sensors \& Actuators: B. Chemical 86(2-3), 198-208.

Gould, G.W., Russell, N.J., Major, New and emerging food poisoning and food spoilage microorganisms. In: Russell, N.J., Gould, G.W. (Eds.), Food preservatives, p. 380, 2 ed. Kluwer Academic/Plenum Publishers, New York.

Goverd, K.A., Beech, F.W., Hobbs, R.P., Shannon, R., 1979. The occurrence and survival of coliforms and salmonellas in apple juice and cider. J. Appl. Bacteriol 46, 521-530.

Grossi, M., Lanzoni, M., Pompei, A., Lazzarini, R., Matteuzzi, D., Ricco, B., 2008. Detection of microbial concentration in ice-cream using the impedance technique. Biosensors and Bioelectronics 23(11), 1616-1623.

Guyer, J., Boettinger, W., Warren, J., McFadden, G., 2003. Phase field modeling of electrochemistry I: Equilibrium. Arxiv preprint cond-mat/0308173.

Haimi-Cohen, Y., Vellozzi, E., Rubin, L., 2002. Initial concentration of Staphylococcus epidermidis in simulated pediatric blood cultures correlates with time to positive results with the automated, continuously monitored BACTEC blood culture system. Journal of clinical microbiology 40(3), 898.

Hall, G.S., Pratt-Rippin, K., Meisler, D.M., Washington, J.A., Roussel, T.J., Miller, D., 1994. Growth curve for Propionibacterium acnes. Current eye research 13(6), 465-466. 
Hayes, M.C., Boor, K., Raw milk and fluid milk products. In: Marth, E.H., Steele, J.L. (Eds.), Applied dairy microbiology, 2 ed. Marcel Dekker, Inc, New York, NY.

Hayes, M.C., Boor, K., 2001. Raw milk and fluid milk products. Applied dairy microbiology, 59-76. Horan, N., 2003. Handbook of water and wastewater microbiology. Academic Pr.

Horstkotte, M.A., Knobloch, J.K.M., Rohde, H., Dobinsky, S., Mack, D., 2004. Evaluation of the BD Phoenix automated microbiology system for detection of methicillin resistance in coagulasenegative staphylococci. Journal of clinical microbiology 42(11), 5041-5046.

Horvath, L.L., George, B.J., Murray, C.K., Harrison, L.S., Hospenthal, D.R., 2004. Direct comparison of the BACTEC 9240 and BaCT/ALERT 3D automated blood culture systems for candida growth detection. Journal of clinical microbiology 42(1), 115-118.

Johnson, J.L., Brooke, C.L., Fritschel, S.J., 1998. Comparison of the BAX for screening/E. coli 0157: H7 method with conventional methods for detection of extremely low levels of Escherichia coli 0157: H7 in ground beef. Applied and Environmental Microbiology 64(11), 4390. Jorgensen, J.H., Ferraro, M.J., 1998. Antimicrobial susceptibility testing: general principles and contemporary practices. Clinical infectious diseases 26(4), 973.

Juffs, H., Deeth, H.C., 2007. Scientific Evaluation of Pasteurisation for Pathogen Reduction in Milk and Milk Products. Food Standards Australia New Zealand 2007, Canberra, Australia.

Kemeny, D., Challacombe, S.J., 1991. ELISA and other solid phase immunoassays: theoretical and practical aspects. Wiley.

Kinnunen, P., Sinn, I., McNaughton, B.H., Newton, D.W., Burns, M.A., Kopelman, R., 2011. Monitoring the growth and drug susceptibility of individual bacteria using asynchronous magnetic bead rotation sensors. Biosensors and Bioelectronics 26(5), 2751-2755.

Kirov, S.M., Hui, D.S., Hayward, L.J., 1993. Milk as a potential source of Aeromonas gastrointestinal infection. Journal of Food Protection 56(4), 306-312.

König, C., Schwank, S., Blaser, J., 2001. Factors compromising antibiotic activity against biofilms of Staphylococcus epidermidis. European Journal of Clinical Microbiology \& Infectious Diseases 20(1), 20-26.

Körner, H., Zumft, W.G., 1989. Expression of denitrification enzymes in response to the dissolved oxygen level and respiratory substrate in continuous culture of Pseudomonas stutzeri. Applied and environmental microbiology 55(7), 1670-1676.

Koutsoumanis, K., Giannakourou, M.C., Taoukis, P.S., Nychas, G.J.E., 2002. Application of shelf life decision system (SLDS) to marine cultured fish quality. International journal of food microbiology 73(2-3), 375-382.

Kumar, A., Roberts, D., Wood, K., Light, B., Parrillo, J., Sharma, S., Suppes, R., Feinstein, D., Zanotti, S., Taiberg, L., 2006. Duration of hypotension before initiation of effective antimicrobial therapy is the critical determinant of survival in human septic shock*. Critical care medicine 34(6), 1589.

Lim, D.V., 2002. Growth and Control of Growth. Microbiology, 3rd Edition ed. Kendall Hunt.

Liu, R.H., Yang, J., Lenigk, R., Bonanno, J., Grodzinski, P., 2004. Self-contained, fully integrated biochip for sample preparation, polymerase chain reaction amplification, and DNA microarray detection. Analytical Chemistry 76(7), 1824-1831.

Locke, J.B., Hilgers, M., Shaw, K.J., 2009. Novel ribosomal mutations in Staphylococcus aureus strains identified through selection with the oxazolidinones linezolid and torezolid (TR-700). Antimicrobial agents and chemotherapy 53(12), 5265-5274.

Madden, R., Gilmour, A., 1995a. Impedance as an alternative to MPN enumeration of coliforms in pasteurized milks. Letters in applied microbiology 21(6), 387-388. 
Madden, R.H., Gilmour, A., 1995b. Impedance as an alternative to MPN enumeration of coliforms in pasteurized milks. Letters in applied microbiology 21(6), 387-388.

Malmberg, C.G., Maryott, A.A., 1950. Dielectric constants of aqueous solutions of dextrose and sucrose. Journal of Research of the National Bureau of Standards 45(4), 299-303.

Mancini, N., Carletti, S., Ghidoli, N., Cichero, P., Burioni, R., Clementi, M., 2010. The Era of Molecular and Other Non-culture-based Methods in the Diagnosis of Sepsis. Clinical Microbiology Reviews 23(1), 235-251.

Manning, M., 2006. claro changes the game of blood analysis. . Tampa bay business journal

Mead, P.S., Slutsker, L., Dietz, V., McCaig, L.F., Bresee, J.S., Shapiro, C., Griffin, P.M., Tauxe, R.V., 2000. Food-related illness and death in the United States. Journal of Environmental Health 62(7).

Meland, A.-K., 2007. Impedance Diagrams of the electrodes in the polymer electrolyte membrane fuel cell, PhD Thesis. Department of Chemistry. Norwegian University of Science and Technology, Trondheim.

Melkikh, A.V., Seleznev, V.D., 2007. Models of active transport of neurotransmitters in synaptic vesicles. Journal of Theoretical Biology 248(2), 350-353.

Michael T. Madigan, J.M.M., David A. Stahl, David P. CLark, 2006. Brocks Biology of Microorganisms.

Morello, J.A., Leitch, C., Nitz, S., Dyke, J.W., Andruszewski, M., Maier, G., Landau, W., Beard, M.A., 1994. Detection of bacteremia by Difco ESP blood culture system. Journal of Clinical Microbiology 32(3), 811-818.

Nandi, N., Bagchi, B., 1998. Anomalous dielectric relaxation of aqueous protein solutions. The Journal of Physical Chemistry A 102(43), 8217-8221.

Nandi, N., Bhattacharyya, K., Bagchi, B., 2000. Dielectric relaxation and solvation dynamics of water in complex chemical and biological systems. ChemInform 31(34), no-no.

Noble, R.T., Weisberg, S.B., 2005. A review of technologies for rapid detection of bacteria in recreational waters. Journal of water and health 3(4), 381-392.

Oblinger, J., Koburger, J., 1975. Understanding and teaching the most probable number technique. Journal of Milk and Food technology 38(9), 540-545.

Pearson, J.P., Passador, L., Iglewski, B.H., Greenberg, E., 1995. A second N-acylhomoserine lactone signal produced by Pseudomonas aeruginosa. Proceedings of the National Academy of Sciences 92(5), 1490.

Peplow, M.O., Correa-Prisant, M., Stebbins, M.E., Jones, F., Davies, P., 1999. Sensitivity, Specificity, and Predictive Values of ThreeSalmonella Rapid Detection Kits Using Fresh and Frozen Poultry Environmental Samples versus Those of Standard Plating. Applied and environmental microbiology 65(3), 1055-1060.

Poortinga, A., Bos, R., Busscher, H., 1999a. Measurement of charge transfer during bacterial adhesion to an indium tin oxide surface in a parallel plate flow chamber. Journal of microbiological methods 38(3), 183-189.

Poortinga, A.T., Bos, R., Busscher, H.J., 1999b. Measurement of charge transfer during bacterial adhesion to an indium tin oxide surface in a parallel plate flow chamber. Journal of microbiological methods 38(3), 183-189.

Pridmore, A., Silley, P., 2001. RAPID DETECTION OF ENTEROBACTERIACEAE IN DEHYDRATED FOODS USING IMPEDANCE MICROBIOLOGY: THE CRITICAL ROLE OF THE PRE-ENRICHMENT PHASE. In: clark, S.A. (Ed.), Rapid detection assays for food and water, p. 152. The Royal Society of chemistry, Cambridge, UK. 
Puttaswamy, S., Lee, B.D., Sengupta, S., 2011. Novel Electrical Method for Early Detection of Viable Bacteria in Blood Cultures. Journal of clinical microbiology 49(6), 2286-2289.

Puttaswamy, S., Sengupta, S., 2010a. Rapid detection of bacterial proliferation in food samples using microchannel impedance measurements at multiple frequencies. Sensing and Instrumentation for Food Quality and Safety, 1-11.

Puttaswamy, S., Sengupta, S., 2010b. Rapid detection of bacterial proliferation in food samples using microchannel impedance measurements at multiple frequencies. Sensing and Instrumentation for Food Quality and Safety 4(3), 108-118.

Radke, S.M., Alocilja, E.C., 2004. Design and fabrication of a microimpedance biosensor for bacterial detection. IEEE sensors journal 4(4), 434-440.

Radke, S.M., Alocilja, E.C., 2005. A high density microelectrode array biosensor for detection of E. coli O157: H7. Biosensors and Bioelectronics 20(8), 1662-1667.

Riedel, S., Eisinger, S.W., Dam, L., Stamper, P.D., Carroll, K.C., 2011. Comparison of BD Bactec Plus Aerobic/F medium to VersaTREK Redox 1 blood culture medium for detection of Candida spp. in seeded blood culture specimens containing therapeutic levels of antifungal agents. Journal of Clinical Microbiology 49(4), 1524-1529.

Rosner, B.A., 2006. Discrete Probability Distributions. In: Rosner, B. (Ed.), Fundamentals of biostatistics, p. 868, 6 ed. Duxbury Press, California.

Ruan, C., Yang, L., Li, Y., 2002. Rapid detection of viable Salmonella typhimurium in a selective medium by monitoring oxygen consumption with electrochemical cyclic voltammetry. Journal of Electroanalytical Chemistry 519(1-2), 33-38.

Russell, S.M., 2000. Comparison of the traditional three-tube most probable number method with the Petrifilm, SimPlate, BioSys optical, and Bactometer conductance methods for enumerating Escherichia coli from chicken carcasses and ground beef. Journal of Food Protection 63(9), 1179-1183.

S. Whittier, S.A.M., R.C. Huard, P. Della - Latta, 2006. Evaluation of the BD Phoenix Automated Microbiology System for Antibiotic Susceptibility Testing of Streptococcus pneumoniae. General Meeting of the American Society for Microbiology (ASM), Orlando, FL.

Salama, I.A., Koch, G.G., Tolley, D.H., 1978. On the estimation of the most probable number in a serial dilution experiment. Communications in Statistics-Theory and Methods 7(13), 1267-1281.

Schwan, H.P., Foster, K.R., 1980. RF-field interactions with biological systems: electrical properties and biophysical mechanisms. Proceedings of the IEEE 68(1), 104-113.

Sengupta, S., Battigelli, D., Chang, H., 2006a. A micro-scale multi-frequency reactance measurement technique to detect bacterial growth at low bio-particle concentrations. Lab on a Chip 6(5), 682-692.

Sengupta, S., Battigelli, D.A., Chang, H.C., 2006b. A micro-scale multi-frequency reactance measurement technique to detect bacterial growth at low bio-particle concentrations. Lab on a Chip 6(5), 682-692.

Sengupta, S., Gordon, J.E., Chang, H.-C., 2010. Microfluidic Diagnostic Systems for the Rapid Detection and Quantification of Pathogens. In: Finehout, E. (Ed.).

Sengupta, S., Mahmud, G., Chiou, D., Ziaie, B., Barocas, V., 2005a. Application of the lag-afterpulsed-separation (LAPS) flow meter to different protein solutions. The Analyst 130(2), 171-178.

Sengupta, S., Mahmud, G., Chiou, D.J., Ziaie, B., Barocas, V.H., 2005b. Application of the lagafter-pulsed-separation (LAPS) flow meter to different protein solutions. Analyst 130(2), 171178.

Sengupta, S., Ziaie, B., Barocas, V., 2004a. Lag-after-pulsed-separation microfluidic flowmeter for biomacromolecular solutions. Sensors and Actuators B: Chemical 99(1), 25-29. 
Sengupta, S., Ziaie, B., Barocas, V.H., 2004b. Lag-after-pulsed-separation microfluidic flowmeter for biomacromolecular solutions. Sensors \& Actuators: B. Chemical 99(1), 25-29.

Skarstad, K., Steen, H.B., Boye, E., 1985. Escherichia coli DNA distributions measured by flow cytometry and compared with theoretical computer simulations. Journal of bacteriology 163(2), 661-668.

Skjerve, E., Rørvik, L., Olsvik, O., 1990. Detection of Listeria monocytogenes in foods by immunomagnetic separation. Applied and environmental microbiology 56(11), 3478-3481.

Smith, J., Serebrennikova, Y., Huffman, D., Leparc, G., García Rubio, L., 2008. A new method for the detection of microorganisms in blood cultures: Part I. Theoretical analysis and simulation of blood culture processes. The Canadian Journal of Chemical Engineering 86(5), 947-959.

Soini, E., Hemmilä, I., 1979. Fluoroimmunoassay: present status and key problems. Clinical chemistry 25(3), 353-361.

Struelens, M., 2010. Detection of microbial DNAemia: does it matter for sepsis management? Intensive care medicine 36(2), 193-195.

Sule, A., Ahmed, Q.U., Samah, O.A., Omar, M.N., 2011. Bacteriostatic and bactericidal activities of Andrographis paniculata extracts on skin disease causing pathogenic bacteria. J Med Plant Res 5(1), 7-14.

Sutton, S., February, 2010. The Most probable Number (MPN) Method. Pharmaceutical Microbiology Forum Newsletter 16 (2), 2 - 9.

Swaminathan, B., Feng, P., 1994. Rapid detection of food-borne pathogenic bacteria. Annual Reviews in Microbiology 48(1), 401-426.

Sy, A., Giraud, E., Jourand, P., Garcia, N., Willems, A., De Lajudie, P., Prin, Y., Neyra, M., Gillis, M., Boivin-Masson, C., 2001. Methylotrophic MethylobacteriumBacteria Nodulate and Fix Nitrogen in Symbiosis with Legumes. Journal of Bacteriology 183(1), 214-220.

Szita, G., Gyenes, M., Soos, L., Retfalvi, T., Bekesi, L., Csiko, G., Bernath, S., 2007. Detection of Pseudomonas aeruginosa in water samples using a novel synthetic medium and impedimetric technology. Letters in applied microbiology 45(1), 42-46.

Teratanavat, R., Hooker, N.H., 2004. Understanding the characteristics of US meat and poultry recalls: 1994-2002. Food control 15(5), 359-367.

Tewari, G., Juneja, V.K., ebrary Inc., 2007. Advances in thermal and non-thermal food preservation. 1st ed. Blackwell Pub., Ames, lowa.

Thorpe, T.C., Wilson, M., Turner, J., DiGuiseppi, J., Willert, M., Mirrett, S., Reller, L., 1990. BacT/Alert: an automated colorimetric microbial detection system. Journal of clinical microbiology 28(7), 1608-1612.

Varshney, M., Li, Y., 2008. Double interdigitated array microelectrode-based impedance biosensor for detection of viable Escherichia coli 0157: H7 in growth medium. Talanta 74(4), 518-525.

Watanakunakorn, C., 1983. In vitro activity of ceftriaxone alone and in combination with gentamicin, tobramycin, and amikacin against Pseudomonas aeruginosa. Antimicrobial agents and chemotherapy 24(2), 305-306.

Wiegand, I., Hilpert, K., Hancock, R., 2008. Agar and broth dilution methods to determine the minimal inhibitory concentration (MIC) of antimicrobial substances. Nature Protocols 3(2), 163175.

Woods, G.L., Fish, G., Plaunt, M., Murphy, T., 1997. Clinical evaluation of difco ESP culture system II for growth and detection of mycobacteria. Journal of clinical microbiology 35(1), $121-$ 124. 
Wrenn, B.A., Venosa, A.D., 1996. Selective enumeration of aromatic and aliphatic hydrocarbon degrading bacteria by a most-probable-number procedure. Canadian journal of microbiology 42(3), 252-258.

Yagupsky, P., Nolte, F., 1990. Quantitative aspects of septicemia. Clinical Microbiology Reviews 3(3), 269.

Yang, L., Banada, P.P., Liu, Y.S., Bhunia, A.K., Bashir, R., 2005. Conductivity and pH dual detection of growth profile of healthy and stressed Listeria monocytogenes. Biotechnology and bioengineering 92(6), 685-694.

Yang, L., Bashir, R., 2007. Electrical/electrochemical impedance for rapid detection of foodborne pathogenic bacteria. Biotechnology Advances.

Yang, L., Bashir, R., 2008. Electrical/electrochemical impedance for rapid detection of foodborne pathogenic bacteria. Biotechnology Advances 26(2), 135-150.

Yang, L., Li, Y., Erf, G.F., 2004a. Interdigitated array microelectrode-based electrochemical impedance immunosensor for detection of Escherichia coli 0157: H7. Anal. Chem 76(4), 11071113.

Yang, L., Li, Y., Griffis, C.L., Johnson, M.G., 2004b. Interdigitated microelectrode (IME) impedance sensor for the detection of viable Salmonella Typhimurium. Biosensors and Bioelectronics 19(10), 1139-1147.

Zafari, Y., Martin, W.J., 1977. Comparison of the BACTOMETER microbial monitoring system with conventional methods for detection of microorganisms in urine specimens. Journal of Clinical Microbiology 5(5), 545-547. 


\section{VITA}

Sachidevi Puttaswamy was born in Bangalore, India to Mr. H.C. Puttaswamy and H. Gowramma as a second child. She did her schooling in Bangalore, India. She obtained her Bachelors of Engineering degree in Biotechnology from the MS Ramaiah Institute of Technology in Bangalore, India under Visvesvaraya Technological University. She joined the University of Missouri at Columbia, MO to pursue her Doctorate of Philisophy degree in Biological Engineering in Fall 2007. Her work has mainly been in the field of developing microbial detection and quantification systems for medical and industrial applications.

She married Sagar Kishore Gupta during the course of her PhD. S. Puttaswamy enjoys dancing, traveling and cooking in her spare time. 